$70-26,392$

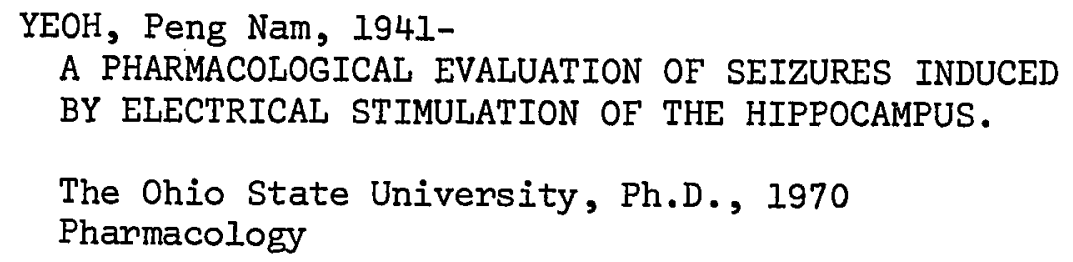

University Microfilms, A XEROX Company , Ann Arrbor, Michigan 


\title{
A PHARMACOLOGICAL EVALUATION OF SEIZURES INDUCED BY ELECTRICAL STIMULATION OF THE HIPPOCAMPUS
}

\section{DISSERTATION}

\section{Presented in Partial Fulfillment of the Requirements for the Degree Doctor of Philosophy in the Graduate School of the Ohio State University}

\section{By}

\author{
Peng Nam Yeoh, B. Pharm., M.Sc.$$
* * * * * *
$$

The Ohio State University

1970

Approved by

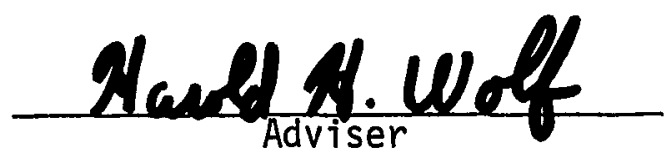

College of Pharmacy 


\section{ACKNOWLEDGMENTS}

I wish to thank Dr. Harold H. Wolf for his advice, help and encouragement. The critical appraisal of the manuscript by Drs. Allan M. Burkman, Michael C. Gerald, Popat N. Patil and John W. Nelson and their helpful suggestions are appreciated. I am also grateful to the Fulbright-Hays program and the College of Pharmacy of The Ohio State University for making this work possible. 


\section{VITA}

December 16, 1941 . . Born - Mentakab, Pahang, Malaysia

1964. . . . . . B. Pharm., University of Singapore, Singapore

1966. ....... M. Sc., The Ohio State University, Columbus Ohio

1967. . . . . . Teaching Assistant, Division of Pharmacology, College of Pharmacy, The Ohio State University, Columbus, Ohio

1967-1970 .... . Research Associate, Division of Pharmacology, College of Pharmacy, The Ohio State University, Columbus, Ohio

\section{PUBLICATIONS}

Yeoh, P.N. and Wolf, H.H. : Effects of some adrenergic agents on low frequency electroshock seizures. J. Pharm. Sci. 57: 340, 1968.

Yeoh, P.N. and Wolf, H.H. : The effect of some adrenergic agents on electrically induced hippocampal seizures. In : Abstracts symposia and contributed papers presented to the A.Ph.A Academy of Pharmaceutical Sciences at 116th. Annual Meeting of the American Pharmaceutical Association at Montreal. 105, 1969.

Yeoh, P.N. and Wolf, H.H. : A pharmacological evaluation of seizures induced by electrical stimulation of the hippocampus. In Press. 


\section{CONTENTS}

Page

ACKNOWLEDGMENTS . . . . . . . . . . . . . . . i i

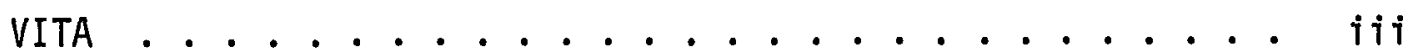

LIST OF TABLES. . . . . . . . . . . . . . . . . vi vi

LIST OF ILLUSTRATIONS ................... . vi

Chapter

I. GENERAL INTRODUCTION. .............. 1

A. Literature Review

B. Basis for the Present Study

C. Statement of the Problem

II. GENERAL PROCEDURES. . . . . . . . . . . . 18

III. EFFECT OF PROTOTYPE ANTICONVULSANTS ON SEIZURES

INDUCED BY ELECTRICAL STIMULATION OF THE HIPPOCAMPUS. .

A. Introduction

B. Methods

C. Results and Discussion

IV. THE IMPORTANCE OF ADRENERGIC TONE ON SEIZURES

INDUCED BY ELECTRICAL STIMULATION OF THE HIPPOCAMPUS. .

A. Introduction

B. Methods

C. Results

D. Discussion

V. GENERAL DISCUSSION. . . . . . . . . . . 153

VI. SUMMARY AND CONCLUSIONS .............. 160 


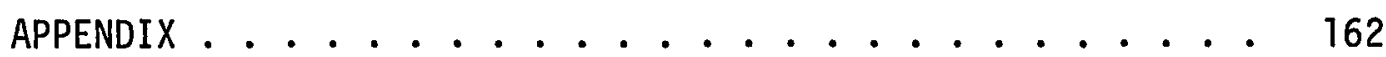

A. Electrode Implantation

B. Verification of Electrode Site and Tissue Damage

C. Histochemical Assay for Norepinephrine

D. Radiometric Assay for Norepinephrine

BIBLIOGRAPHY . . . . . . . . . . 173 


\section{LIST OF TABLES}

Table

Page

1. Neurotoxicity of drugs.............. 20

2. The Stability of Rat Resistance . . . . . . . . 35

3. Effects of Reserpine, $\alpha$-Methy1-p-Tyrosine and DL-threo-DOPS on Norepinephrine Levels in Different Brain Areas in the Rat . . . . . . . . 104

4. Effects of Reserpine, $\alpha$-Methyl-p-Tyrosine and DL-threo-DOPS on Norepinephrine Levels in Different Brain Areas in the Rat . . . . . . . . 105 


\section{LIST OF ILLUSTRATIONS}

Figure

Page

1. Circuit diagram .............. 31

2. Stability of seizure threshold over time. . . . . 37

3. Effect of diphenylhydantoin on seizure threshold ............... 44

4. Effect of trimethadione on seizure threshold. . . 47

5. The importance of adrenergic tone to seizure

6. Effect of phentolamine on seizure threshold . . . 108

7. Effect of phenoxybenzamine on seizure threshold . . 111

8. Effect of propranolol on seizure threshold. . . . 114

9. Effect of pronethalol on seizure threshold. . . . 117

10. Effect of sotalol (MJ1999) on seizure threshold. . 119

11. Effect of INPEA on seizure threshold. . . . . . 121

12. Effect of phenoxybenzamine and phentolamine on seizure thresholds in animals with al tered catecholamine levels............ 145

13. A representative norepinephrine standard curve. . 171

Plate

I Minimal and maximal extent of the brain lesions projected on frontal sections (A-D) and saggital section (E) at the stated distances from frontal and saggital zeros, respectively ....... 
II. Histochemical studies of drug effects on central norepinephrine neurons ..............

III. Histochemical studies of drug effects on central. norepinephrine neurons . . . . . . . . . .

IV. Histochemical studies of drug effects on central. norepinephrine neurons . . . . . . . . .

V. Histochemical studies of drug effects on central norepin ephrine neurons . . . . . . . . . .

VI. Histochemical studies of drug effects on central norepinephrine neurons ............. 


\section{GENERAL INTRODUCTION}

\section{A. Literature Review}

The history of the seizure state is as old as man. In the past, patients suffering from epileptic convulsions were thought to be possessed by devine or evil forces. Hippocrates was, perhaps, the first investigator to associate the disease with a disfunction of the brain. At present, we recognize that seizures are varied in type and can result from "occasional, sudden, excessive, rapid and local discharges of gray matter" (for reviews see Streeter, 1931; Penfield and Erickson, 1941 ; Temkin, 1945; Tower, 1960; Mi11 ichap, 1965).

The classification of seizures clinically is based on the patient's abnormal electroencephalogram (EEG) and the presence and nature of subject-auras. Seizures are generally treated by selective drug therapy and/or surgical removal of the epileptogenic focus (foci).

Over the last 3 decades, a large number of laboratory tests have been developed for routine screening of potential anticonvulsant agents. This development has contributed to the availability of drugs for the management of epileptic seizures. In the laboratory, electricallyinduced seizures were employed by some investigators (Putnum and Merritt, 1937; Merritt and Putnum, 1938), whose work resulted in the introduction of diphenylhydanto in for the treatment of grand mal epilepsy. Similar laboratory studies yielded trimethadione as an effective agent 
against petit mal epilepsies (Everett and Richards, 1944; Goodman et al., 1946; Richards and Everett, 1946; Richards and Perlstein, 1946).

Electrical stimuli may be applied via corneal electrodes to induce a minimal electroshock seizure, or a maximal hind limb "flexor-extensor" seizure (Goodman et al., 1949; Swinyard, 1949; Swinyard et al., 1952). Direct electrical stimulations of specific cortical or subcortical regions have also been employed in producing experimental seizures in the laboratory (Millichap, 1965; Swinyard, 1969). Stimuli other than electrical current have also been used. For example, chemicals like metrazol, methionine sulfoximine, thiosemicarbazide, strychnine and picrotoxin have been used and are generally administered systemically. Other compounds (e.g., penicillin, metals, alumina cream) can induce the formation of chronic epileptogenic foci when placed directly into brain substance. Sensory stimulation has also been employed to induce audiogenic seizures in mice and rats (Plotnikoff and Green, 1957; Plotnikoff, 1958; Fink and Swinyard, 1959) and light-induced seizures in rabbits (Barnes, 1954) and in baboons (Killam, 1967; 1969; Killam et al., 1967). Still other techniques involve manipulations of body metabolism, e.g., imbalance of water and electrolytes in vivo, induced by administration of vasopressin or glucose (Swinyard, 1949; Swinyard et al., 1952), by carbon-dioxide withdrawal (Woodbury et al., 1952) and hyperthermia (Mi17ichap, 1958; Millichap et al., 1960; Millichap, 1965). Furthermore, seizures have also been produced by hypophysectomy, or administration of insulin (DeSalva, 1962; 1963; Kreindler, 1965).

The majority of laboratory studies use intact animals. Mice and rats are the most common animals employed, but cats, hamsters, rabbits 
and baboons have also been studied (Swinyard et al., 1952; Barnes, 1954; Esplin, 1957; Killam, 1967). In addition, other biological systems have also been used. These include the response of single neurons in different brain areas, isolated nerves, nerve-muscle preparations, tissue slices, cortical slabs and the spinal cord (Swinyard, 1969). Seizures produced in the laboratory are generally classified into minimal and maximal seizures. A minimal seizure is characterized by a "stun" response (a period of immobility and "frozen" behavior), facial clonus (including eye twitching and rhythmic jaw movements) and continuous flexion and extension of the fore 1 imbs. In addition, when seizures are induced by sound, there is present a phase of "wild running" (Millichap, 1965). A maximal seizure occurs when tonic flexion and extension of both the fore and hind limbs are exhibited. This can be followed by unconsciousness and death due to respiratory failure (Millichap, 1965).

Both minimal and maximal seizures may be induced by the same type of stimulus by varying its strength, duration and the route of application. Thus, by changing the parameters of the electrical pulse, either minimal or maximal seizures are elicited. Further, subcutaneous injections of metrazol produce minimal seizures, while maximal seizures result from intravenous administration of the drug.

Minimal and maximal seizures do not respond in the same way to anticonvulsant agents. For instance, diphenylhydantoin has poor ability to raise the threshold of animals to minimal seizures (Goodman et al., 1949), but is very effective in abolishing the hind 1 imb tonic extension of maximal electroshock seizures (Toman et al., 1946; Toman, 1965). 
On the contrary, trimethadione readily elevates the thresholds of animals to minimal seizures, but has no effect on the hind limb tonic extensor component of maximal seizures, except at high doses (Toman et al., 1946; Swinyard et al., 1952; Brown et al., 195i). These two compounds also differ in their effects on synaptic transmission, as indicated by studies utilizing the spinal cord and the stellate ganglion (Esplin, 1957; Espl in and Curto, 1957). DiphenyThydantoin reduces polysynaptic potentials slightly and deepens presynaptic depression following impulse transmission. It also reduces synaptic transmission during repetitive stimulation. The drug has a pronounced ability to suppress post-tetanic potentiation (PTP). Trimethadione has no effect on monosynaptic transmission of single impulses. However, it depresses polysynaptic activity slightly. The drug has no action on PTP, but it markedly deepens depression following an impulse, thus reducing transmission after repetitive stimulation. These observations suggest that diphenylhydantoin and trimethadione alleviate seizures by different mechanisms. In addition, the phenomenon of PTP is probably involved in the genesis of maximal seizures.

Post-tetanic potentiation is a general synaptic phenomenon. It occurs when repetitive stimulation of an afferent fiber results in a marked increase in efferent response compared to the pre-tetanic discharge. The phenomenon has been studied in spinal polysynaptic pathways, neuromuscular junctions, in several pathways in the brain and in stellate ganglion. It has been suggested that PTP results from an increase in the amount of neurotransmitter released following tetanization (see review by Esplin and Zablocka, 1969). The degree 
of synaptic transmission following repetitive stimulation is controlled by the interaction of an excitatory and an inhibitory component at the junction. The excitatory component is the potentiating process, while the inhibitory component consists of presynaptic and postsynaptic depression after impulse discharge and the presence of synaptic inhibition. Post-tetanic potentiation, when it occurs, is long-lasting and will increase synaptic transmission by overwhelming the inhibitory component at the synapse.

Epileptogenic neurons can discharge at very high rates, up to 1,000/sec. (Schmidt et a1., 1959; Ward, 1966) and can therefore give rise to PTP. Since neuronal network in the brain generally contain many convergent junctions which produce postsynaptic discharge only following temporal summation, PTP can "open" up new pathways which in turn bombard other convergent synapses, leading to the spread of the abnormal electrical discharge (see review by Esplin and Zablocka, 1969). The demonstration that PTP is depressed by diphenylhydantoin, which also suppresses the spread of seizure discharge in the brain, indicates that PTP probably enhances seizure spread (Toman et a7., 1946; Toman and Goodman, 1948). Further, since diphenylhydanto in is effective against grand mal epilepsy as well as the tonic phase of maximal electroshock seizures, it is likely that PTP is also involved in these conditions. On the other hand, trimethadione has no effect on PTP and seizure spread (Toman et al., 1946; Toman and Goodman, 1948). The drug does not change the tonic phase of maximal electroshock seizures but readily suppresses thresholds to minimal seizures. In addition, it 
is effective against petit mal epilepsies clinically. This information suggests that PTP is probably not involved in the genesis of petit mal epilepsies or in the production of minimal seizures. It has been postulated that petit mal seizures originate in a nonspecific thalamic system with a recurrent cortico-thalamic loop for excitation (see reviews by Kreindler, 1965 and by Toman, 1965). This system apparently does not require PTP for its maintenance.

Activation of an epileptogenic focus probably occurs by explosive self-recruitment of neurons in the central nervous system (Fessard, 1958). A variety of factors can trigger this event. Among these are lowered threshold to neuronal excitation, structure arrangements which facilitate synaptic and ephaptic transmission, changes of recovery cycle and failure of inhibitory mechanisms. These factors are altered by the application of an external stimulus (electrical, chemical or sensory) and by variations in cerebral metabolism and blood circulation (see review by Kreindler, 1965).

Since the central excitable state is a result of interactions of various neurotransmitters in the brain, these transmitters are implicated in the susceptibility of animals to seizures. Among these are acetylchol ine (ACh), gamma-aminobutyric acid (GABA), serotonin (5-HT), and catecholamines (norepinephrine and dopamine). These neurohumors are generally synthesized in neurons and stored in synaptic vesicles in nerve endings (Maynert and Kuriyama, 1964; De Robertis, 1966; Whittaker, 1966).

Evidence for the presence of central cholinergic neurons is based on histochemical studies of acetylcholinesterase (AChE) distribution in 
the brain (Koelle, 1954). Acetylcholinesterase is present in almost all areas of the rat brain, with high concentrations in the basal ganglia (caudate-putamen) and in regions where cholinergic peripheral fibers originate (e.g., somatic motor nuclei, nuclei of preganglionic autonomic fibers).

Acetylcholine generally increases the susceptibility of animals to seizures when administered by intracarotid, intraventricular and intracisternal injections. When locally applied to different brain areas, the compound induces maximal seizures or seizure discharges in the electroencephalogram (EEG) of the test animals (Feldberg, 1945; Bornstein, 1946; Feldberg and Sherwood, 1954a). Such an action may be related to the ability of ACh to depress certain cortical neurons (Phillis and York, 1968), which may be participating in the inhibition of seizure genesis (Phillip, 1956; Li and Chou, 1960).

The metabolism of ACh is abnormal during the period between seizure episodes in animals and men susceptible to seizures (Tower, 1958). Cholinesterase activity is raised and there is an inability to bind ACh.

A certain level of ACh has to be present for seizures to occur (McIIwain, 1955). In McIlwain's study, seizures were arrested when endogenous ACh levels were reduced to $40 \%$ of normal. Similar results were obtained by other workers who examined metrazol (Crossland, 1953) and picrotoxin seizures (Stone et al., 1945).

The level of ACh in vivo may be increased by prevention of ACh metabolism with anticholinesterases (Koelle, 1965). Such compounds, e.g., neostigmine and physostigmine produce seizure discharges in the 
EEG when administered intraventricularly (Feldberg and Sherwood, 1954b). The long-acting antichol inesterase, di isopropyl phosphorofluoridate (DFP) also induces seizures (Himwich et al., 1950).

The effects of ACh at effector sites can be blocked by atropine (Innes and Nickerson, 1965). When administered systemically or intracerebrally, atropine readily inhibits seizures induced by ACh or AChE inhibitors. However, it is ineffective against seizures induced by nicotine or metrazol and has only a slight ability to raise electroshock seizure thresholds (Zablocka, 1963; Longo, 1966). Similar findings were obtained by the same investigators using benzactyzine a synthetic anticholinergic compound. Acetylcholine synthesis is stimulated following picrotoxin and metrazol (McLennan and Elliott, 1951). Subsequent to ACh release there is an increase in permeability of the postsynaptic membrane to sodium and potassium ions, resulting in a reduction of the resting membrane potential. This lowers the threshold to neuronal excitation and tends to facilitate the manifestation of seizures (Woodbury, 1958a; 1958b).

In summary, ACh increases seizure susceptibility - an action subjected to antagonism by prototype cholinergic blocking agents, e.g., atropine and benzactyzine.

Another neurotransmitter implicated in the precipitation of seizures is gamma-aminobutyric acid (GABA). This substance is probably an inhibitory transmitter in the central nervous system. It is synthesized by the decarboxylation of glutamate - a reaction which is catalyzed by glutamate decarboxylase (GAD). Gamma-aminobutyric acid 
participates in the transamination of $\alpha$-ketoglutarate to succinic semialdehyde. Both $\alpha$-ketoglutarate and succinic semialdehyde can be oxidized by various brain preparations and can support oxidative phosphorylation (McKhann and Tower, 1959).

When GABA is administered intraventricularly into cats, it reduces the duration of, and increases the threshold to electricallyinduced hippocampal seizures (DasGupta et al., 1958). When injected intracisternally into animals, the compound lowers their "seizure severity score" to audiogenic and metrazol-induced seizures (Schlesinger et al., 1969).

Total brain levels of GABA can be raised by inhibition of the $\alpha$ ketoglutarate transamination process with compounds like hydroxylamine and amino-oxyacetic acid (AOAA). These two compounds have slight anticonvulsant action (Kohli and Kishor, 1965; Kuriyama et al., 1966). Amino-oxyacetic acid also prevents barbiturate withdrawal seizures (Essig, 1968), thiosemicarbazide, methionine-induced seizures (DaVanzo et al., 1961) and audiogenic seizures (Schlesinger et al., 1968a).

Further support for the involvement of GABA in seizure excitability comes from studies using diets which are deficient in pyridoxine. This compound is an essential co-factor in the decarboxylation of glutamate. Such diets increase the susceptibility of animals to audiogenic seizures (Lyons et al., 1958; Coleman and Schlesinger, 1965).

When exposed to seizures induced by high oxygen pressure, the brain GABA levels in rats are significantly lower than in normal animals. This decrease occurs prior to the onset of seizures (Wood and 
Watson, 1963). Moreover, in several mammalian species good correlation is obtained between the reduction of GABA concentrations and shorter latencies to seizures (Wood et al., 1967; 1969). By inhibition of GAD the in vivo concentration of GABA can be reduced. This occurs with treatment of thiosemicarbazide, semicarbazide, isoniazide and 3-deoxypyridoxine phosphate (Killam, 1957; Maynert, 1969). Generally, when the level of GABA is lowered by $40 \%$ or greater, tonicclonic seizures are produced.

Therefore, these observations seem to indicate that GABA concentrations in the brain influence the susceptibility of animals to seizures - an elevation in GABA levels lower seizure excitability and vice versa. However, contradictory data to the above hypothesis have also been reported. For example, Maynert and Kaji (1962) failed to see any change in susceptibility to electrically-induced, metrazol and semicarbazide-induced seizures in mice and rats with elevated GABA concentrations. This was induced by treatment with hydrazine dihydrochloride and hydroxylamine. A possible explanation for this discrepancy was provided by Tapia and Awapara (1967), who examined the incorporation of C14-glutamic acid into GABA in animals treated with glutamic acid- $\gamma$-hydrazide. This compound increased total brain GABA levels, but decreased the incorporation of glutamic acid into GABA at the time of convulsions. Such findings demonstrate that the involvement of GABA in seizure susceptibility may be related to its rate of turnover, rather than to the in vivo brain concentrations of the neurohormone. 
In contrast to GABA, its precursor, glutamate exerts powerful excitatory actions on nervous tissue (Curtis and Watkins, 1965). When injected intracerebrally, intraventricularly or through the carotid artery, glutamate produces convulsions which can be antagonized by GABA (Hayashi, 1952; Tani, 1954; Hayashi and Nagai, 1956).

Serotonin (5-HT) is another neurotransmitter which has been implicated in the regulation of seizure susceptibility. Many drugs that alter catecholamine levels have similar effects on 5-HT levels, e.g., reserpine, tetrabenazine and monoamine oxidase inhibitors (Anden et al., 1969). Both reserpine and tetrabenazine inhibit the uptake of monoamines into granular stores and therefore lower biogenic amine concentrations. Inhibitors of monoamine oxidase prevent the metabolism of 5-HT and cause an elevation of this amine in vivo.

As a result of histochemical studies, central serotoninergic neurons have been identified (Anden et al., 1969). Their cell bodies are located in the medulla oblongata, pons and mesencephalon, while their nerve terminals are distributed in the hypothalamus, the limbic system and the neocortex.

Serotonin is implicated in the precipitation of audiogenic seizures, since susceptible mouse strains have lower $5-\mathrm{HT}$ levels during the period of high seizure risk (Schlesinger et al., 1965). Further, 5-HTP (5-hydroxytryptamine), the precursor of 5-HT protects mice against audiogenic seizures. Reserpine, which lowers 5-HT levels, increases the susceptibility of animals to audiogenic seizures (Schlesinger et al., 1968a; 1968b). In addition, reserpine also in- 
creases seizure susceptibility in audiogenic seizure-prone animals to metrazol or electrically-induced seizures, while 5-HTP has the reverse effect (Schlesinger, 1968b).

Less in and Parkes (1959) evaluated the survival time of mice, administered i.v. metrazol infusions. Both reserpine and tetrabenazine reduced survival time. Pretreatment with iproniazid, a monoamine oxidase inhibitor, antagonized the reserpine and tetrabenazine effect. In the presence of 5-HTP and iproniazid, the survival times to metrazol seizures were prolonged. The time course of metrazol facilitation by reserpine corresponded well to the reduction in brain 5-HT levels, suggesting its involvement in metrazol seizures.

Significant increases in the thresholds to electrically-induced extensor seizures in iproniazid-treated animals with or without reserpine pretreatment are produced following 5-HTP (Chen et al., 1968a). Depletion of 5-HT occurs following treatment with p-chlorophenylalanine, which inhibits its synthes is (Koe and Weissman, 1967). When this compound was investigated for its ability to influence seizure susceptibility, it lowered the threshold to electrically-induced extensor seizures (Chen et al., 1968b).

Although the above studies indicate a $5-\mathrm{HT}$ influence on seizure manifestation, some investigators have reported results which contradict this hypothesis. For example, McGeer et al. (1969) found no significant difference in 5-HT concentrations in brains of seizure susceptible animals compared to resistant animals. Pfeifer and Galambos (1967) examined the effect of $p$-chloroamphetamine on seizure susceptibility in mice and rats. This compound is known to deplete brain 
5-HT without altering catecholamine levels (Fuller et al., 1965). Their data showed that $\mathrm{p}$-chloroamphetamine increased the convulsive threshold in mice with normal 5-HT levels, as well as in rats where the $5-\mathrm{HT}$ concentrations were 10 wered by $40 \%$.

The above studies indicate that the influence of 5-HT on seizure susceptibility is not a simple one. Although there is predominating evidence pointing towards a relationship of high 5-HT level to low seizure susceptibility and low 5-HT level to high seizure susceptibility, data contradicting this hypothesis have also been presented. Perhaps, like the situation with GABA, the rate of $5-\mathrm{HT}$ turnover may be more important in the control of seizure excitability rather than the whole brain level of the hormone.

The responsiveness of an animal to seizures is probably determined by the activities of all the normally occurring neurotransmitters interacting together. Among those discussed thus far, ACh and GABA are perhaps the more important ones involved. However, investigations into the nature of GABA involvement in seizure behavior is at present limited by the comparatively few compounds available to alter its metabolism and disposition in vivo. Over the last decade an extremely large number of studies have been undertaken to establish the influence of catecholamines (norepinephrine and dopamine) on seizure susceptibility. This is also facilitated by the great number of available compounds which are capable of changing catecholamine levels in the intact animal. At present, the data seem to implicate norepinephrine as an inhibitory transmitter in the control of seizure genesis. Such an observation is derived from studies which examined the interactions of 
various adrenergic agents with the susceptibility of animals to different types of experimental seizures. Endogenous catecholamine levels have been altered via direct administration of the parent compound or its amino acid precursor. By changing the amount of synthesis, uptake, storage, metabolism and the availability of the neurotransmitter at the active sites, catecholamines have been shown to alter seizure excitability. A detail literature survey of the existing data will be presented in Chapter IV. Generally, a lack of norepinephrine increases the responsiveness of an animal to seizures. When the concentration of this neurotransmitter is elevated, the test animal tends to be less susceptible to seizures.

In addition to ACh, GABA, 5-HT and catecholamines, other naturaliy occurring neurohumors have also been suggested to influence seizure susceptibility. Among these are substance $P$ and histamine. Substance $P$ is a polypeptide known to be present in the brain (von Euler and Gaddum, 1931) and has been implicated in neurotransmission in the brain. However, there are relatively few studies which examined the effect of this substance on seizure excitability, and so far a clear-cut relationship has not been established (Haefely and Hurlimann, 1962; Lembeck and Zetler, 1962). With histamine, little is known of its central effects at present, although systemic administration of this compound has been shown to produce convulsions (Maynert, 1969).

This literature survey indicates that seizures can be induced by a variety of artificial stimuli. The types of seizures produced are different, in that they vary in pattern, in their responses to anticonvulsant drugs and in their influences on synaptic transmission. 
The genesis of seizures seems to involve different types of electrophysiological phenomena. These in turn may be inter-related to the activities of naturally occurring neurotransmitters like ACh, GABA, 5-HT and catecholamines.

B. Basis for the Present Study Laboratory seizures can be induced by electrical stimulation, chemoshock and sensory stimulation. However, the available methods suffer from many disadvantages. Electroshock methods which employ corneal electrodes require brief restraint of the subject for application of the stimulus. Since brief restraint per se can lower seizure threshold (Swinyard et al., 1962), it can confound the data generated in experiments where animals have to be briefly restrained. Druginduced seizures, on the other hand, are subject to the criticism of possible drug-drug interaction, especially in studies where responses to chemicals are employed to examine seizure mechanisms. Finally, seizures dependent upon sensory stimulation, e.g., audiogenic seizures, are self-limiting, in that such genetically predisposed animals do not maintain their susceptibility (Castellion et al., 1965). The scarcity of the species, Papio Papio, also discourages wide-spread use of spontaneous photic seizures.

From these considerations we can see that a suitable experimental model for the determination of seizure mechanisms is still lacking. The first aim of this study was to design a method to induce seizures in freely moving animals with a physical stimulus. These seizures were induced by electrical stimulation of the hippocampus. For 
comparative purposes, effects of two prototype anticonvulsant agents, diphenylhydantoin and trimethadione, were evaluated.

As indicated before, many investigations have indicated that catecholamines are involved in the regulation of seizure susceptibility. Earlier studies in this laboratory (Yeoh, 1966) showed that propranolol and pronethalol elevated seizure threshold, while phenoxybenzamine had the reverse effect. Reserpine or $\alpha-m m T$ treatment reduced the effects due to pronethalol and phenoxybenzamine but not that of propranolol. These studies show that adrenergic blocking agents alter seizure excitability. Thus, the second aim of this problem was to demonstrate an involvement of catecholamines (especially norepinephrine) in seizures induced by electrical stimulation of the hippocampus and to investigate the effects of adrenergic blocking agents on these seizures. This aspect of the study was undertaken to examine the relationship of alteration of seizure susceptibility to adrenergic receptor blockade.

\section{Statement of the Problem}

A method was designed for producing minimal seizures via electrical stimulation of the right, dorsal hippocampus in the unrestrained rat. The response measured was the current required to produce a minimal seizure. Dose-response curves demonstrating the ability of two prototype anticonvulsants, $\underline{\text { i.e., }}$ diphenylhydantoin and trimethadione, to prevent such seizures were generated. 
Catecholamine involvement in the susceptibility of rats to such seizures was examined using animals treated with reserpine, alphamethyl-p-tyrosine $(\alpha-m T)$ and combined injections of both these drugs. In order to examine the influence of norepinephrine on this behavior, an amino acid precursor of 1-norepinephrine was used to selectively replete 1-norepinephrine in rats previously depleted of catecholamines with treatment of reserpine plus $\alpha-m T$. For this purpose, 3,4-dihydroxyphenylserine was examined for its effect on seizure threshold (Holtz, 1959; Carlsson, 1964; Creveling et al., 1968). These findings were correlated with histochemical and biochemical determinations of norepinephrine in specific brain areas in order to relate seizure threshold alterations with changes of norepinephrine levels in the brain.

Dose-response studies were also conducted to evaluate the effects of two alpha adrenergic blocking agents (phenoxybenzamine and phentolamine) and 5 beta adrenergic blocking compounds (propranolol, pronetha101, sota101, $D(-)$ and $L(+)$ INPEA) on seizure threshold. From the data obtained in these experiments, it was anticipated that the participation of central adrenergic receptors in the genesis of seizures induced by electrical stimulation of the hippocampus could be verified. 


\section{GENERAL PROCEDURES}

The experimental animals were male, albino, Wistar rats (200 to $300 \mathrm{gm}$. ) obtained from Greenacres Laboratories, Amelia, Ohio. Before electrode implantation they were kept in groups of 5 , in stainless steel holding cages $(18 \times 28 \times 56 \mathrm{~cm}$.) with free access to food (Purina rat chow) and water. In addition to observing a $12 \mathrm{hr}$. light cycle, room temperature was maintained at $23^{\circ} \mathrm{C}$.

Except in the neurotoxicity studies, all the animals employed had bipolar electrodes implanted in the right, dorsal hippocampus. Subsequent to surgery these rats were housed individually in plastic cages $(16 \times 25 \times 25 \mathrm{~cm}$.$) with free access to food and water under the$ same light and temperature conditions as described above.

Seizure thresholds were determined between $10 \mathrm{a} . \mathrm{m}$. and $8 \mathrm{p} . \mathrm{m}$. Test and control studies were always conducted simultaneously. This precaution was observed since seizure susceptibility varies slightly throughout the day (Woolley and Timiras, 1962).

The drugs studied were diphenylhydantoin (Parke Davis); propranolol (1-Isopropylamino-3-(1-Naphthoxy)-2-propanol hydrochloride Imperial Chemical Industries); pronethalol (D,L-1-(2'Naphthy1)-2-Isopropy1 aminoethanol hydrochloride, Imperial Chemical Industries); Sotalol (MJ1999, 4-(2-Isopropyl-amino-1-hydroxyethyl) methanesulfonanilide, (Mead Johnson); $D(-)$ INPEA (D(-) 1-(4'-nitropheny1-2-Isopropy1 aminoethanol hydrochloride, Selvi \& Co.); L(+) INPEA (Selvi \& Co.); alpha- 
methyl-tyrosine (Merk, Sharp \& Dohme Research Laboratories); reserpine (Ciba); DL-Dops (H22/72, DL-threo-3,4-dihydroxyphenyl serine, Axel Kistner $A B$ ); phenoxybenzamine hydrochloride (Smith, Kline \& French Laboratories) and phentolamine (regitine mesylate, Ciba).

Al1 drug injections were made intraperitoneally, employing volumes of 0.8 to $3.0 \mathrm{ml} . / \mathrm{kg}$. Diphenylhydantoin, DL-threo-Dops and phenoxybenzamine were suspended in 10\% propylene glycol in saline, while the other compounds were dissolved in saline (sometimes with the aid of an ultrasonic frequency generator). Control animals received the drug vehicle.

In order to determine appropriate doses and the duration of drug activity, pilot studies were conducted based on drug-induced neurotoxicity (Weaver and Miya, 1961). This was defined as the failure of a rat to stay on a rod (rotating at 6 r.p.m.) for 1 min., given 3 trials. Drug-induced neurotoxicity in $50 \%$ of rats (TD50) with $95 \%$ confidence limits was calculated using the method of Litchfield and Wilcoxon (1949). The results are represented in Table 1. 
TABLE 1. Neurotoxicity of Drugs

\begin{tabular}{llll}
\hline \multicolumn{1}{c}{ Drug } & $\begin{array}{c}\text { Mol. } \\
\text { Wt. } \\
\text { (sait) }\end{array}$ & $\begin{array}{l}\text { Time of Peak } \\
\text { Effect (Min.) }\end{array}$ & $\begin{array}{l}\text { Toxic Dose 50 } \\
(x 10-1 \text { mMoles/kg. })\end{array}$ \\
\hline Diphenylhydantoin & 274.25 & 60 & $8.3(5.90-11.60)^{\mathrm{a}}$ \\
Trimethadione & 143.14 & 10 & $32.80(29.50-33.40)$ \\
Propranolo1 & 295.80 & 15 & $1.11(1.04-1.18)$ \\
Pronetha101 & 265.80 & 15 & $1.23(0.99-1.52)$ \\
Sota101 (MJ1999) & 308.80 & 30 & $>8.0^{\mathrm{b}}$ \\
D(-) INPEA & 244.50 & 20 & $2.06(1.35-3.15)$ \\
L(+) INPEA & 244.50 & 20 & $2.08(1.51-2.87)$ \\
Phenoxybenzamine & 340.34 & 90 & $3.25(2.24-4.71)$ \\
Phentolamine & 377.47 & 20 & $1.08(0.58-1.99)^{\mathrm{C}}$ \\
& & & \\
\hline
\end{tabular}

a The figures within parentheses represent $95 \%$ confidence 1 imits .

b Complete toxicity studies for sotalol were not conducted due to 1 imited amount of drug and to its relative non-toxicity. No toxic effect was seen with $8 \times 10^{-1} \mathrm{mMoles} / \mathrm{kg}$. The choice of $30 \mathrm{~min}$. as the time of peak effect was based on observable, gross central nervous system depression.

c None of the test animals failed the rolling-bar test even with a dose of $2 \times 10^{-1}$ mMoles $/ \mathrm{kg}$. Test animals were considered neurotoxic when they exhibited central depression, rhythmic jaw movements and reduced exploratory behavior. 


\section{EFFECT OF PROTOTYPE ANTICONVULSANTS ON SEIZURES \\ INDUCED BY ELECTRICAL STIMULATION OF \\ THE HIPPOCAMPUS}

\section{A. Introduction}

Several techniques to produce seizures in the laboratory animal are available (Millichap, 1965; Killam et al., 1967; Chusid and Kopeloff, 1969; Killam, 1969; Swinyard, 1969). These employ stimuli like electricity, chemicals, sound, light and metabolic changes.

Electrically-induced seizures may be produced by generalized stimulation of the intact animal resulting in either a minimal (clonic) or a maximal (flexor-extensor) seizure. The pattern exhibited is determined by the parameters of the stimulus (Goodman et a1., 1949; Swinyard, 1949; 1969; Swinyard et al., 1952). Further, local electrical stimulation of selected areas of the brain or spinal cord can be used to el icit minimal or maximal seizures (Rosenblueth and Cannon, 1942; Green and Shimamoto, 1953; Kopeloff et al., 1954; Espl in, 1957; Espl in and Curto, 1957; Green et al., 1957; MacLean, 1957a; 1957b; Esplin and Freston, 1960; Roelofs, 1967).

of the chemicals employed to produce seizures in the intact animal, pentylenetetrazol, strychnine and picrotoxin are the commonest. Methionine sulfoximine, thiosemicarbazide, hexaflurodiethylether and MoB6 (4-methoxy-methylpyridoxine hydrochloride) have also been used. The type of seizure induced is determined by the drug, its dose and route of administration (Goodman et al., 1946; Mi11ichap, 1965; Chusid 
and Kopeloff, 1969; Swinyard, 1969). Chronic epileptogenic animals can also result from local stimulation of specific brain areas with irritative agents (e.g., penicillin, tungstic acid gel, alumina cream, metals like cobalt, nickel and antimony). In addition, local freezing of brain regions with ethyl chloride spray or a cold metal rod can generate chronic epileptogenic lesions (Millichap, 1965; Chusid and Kopeloff, 1969; Swinyard, 1969). These latter methods yield acute, intermittent and recurrent seizures which can persist for a period of years.

Sound-precipitated seizures occur in a wide variety of species (e.g., mice, rats, rabbits, guinea pigs and man). Both pure tones and sound of mixed frequencies can be used as the stimulus (Critchley, 1937; Bevan, 1955; Mil1ichap, 1965; Gastaut and Tassinari, 1966). Whether a maximal or a minimal seizure is produced depends on the type, intensity and duration of the stimulus. The susceptibility of animals to audiogenic seizures is genetically determined and by selective inbreeding several strains of animals with a high incidence to such seizures have been developed.

Not so widely used, but also genetically predetermined are light precipitated seizures in the baboon (Killam, 1967, 1969; Killam et al., 1967; Fischer-Williams et al., 1968) and in the rabbit (Barnes, 1954). These photic seizures are either minimal or maximal in type and resemble seizures observed in photic sensitive epileptic patients.

A variety of techniques to manipulate metabolism in the intact animal can be employed to induce seizures in the laboratory. These are 
based on alterations of water or electrolyte (Swinyard, 1949; Swinyard et al., 1952), carbon dioxide withdrawal (Woodbury et al., 1958) hyperthermia (Millichap, 1958; 1965; Millichap et al., 1960) and endocrine ablation or hormone administration (DeSalva, 1962; 1963). These methods produce both minimal and maximal seizures.

The seizures induced in the laboratory include patterns ranging from "stun response", catatonia, hyperkinesia, twitching of the eyelids, clonus of the face, rhythmic movements of the jaws, clonus of the fore limbs to tonic flexion and extension of the hind limbs and death due to respiratory failure. Presence of some or all of the first 7 signs is indicative of a minimal (cionic) seizure, while the manifestation of some or all of the last 3 signs indicates a maximal seizure (Millichap, 1965). These two types of seizures react differently to anticonvulsants and have been used in the screening of such drugs. They have also been employed in studies to elucidate seizure mechanisms (Millichap, 1965; Esplin and Zablocka, 1969; Swinyard, 1969). There is good correspondence between drug effects on experimental seizures and clinical seizures, in that drugs normally effective against maximal seizures protect against grand mal epilepsy, while agents capable of raising thresholds of intact animals to minimal seizures are useful in the control of petit mal epilepsy. The converse is also true, i.e., drugs which do not attenuate maximal seizure pattern do not protect against grand mal epilepsy, while those which are ineffective in raising minimal seizure threshold do not control petit mal epilepsy (Millichap, 1965; Toman, 1965; Swinyard, 1969l. 
Of these two types, minimal seizures are less stressful to the experimental animal compared to maximal seizures and are therefore more suitable as models for determination of seizure mechanisms, especially if such studies are to be conducted in the same animals over a long time.

As discussed earlier, there is lacking a suitable experimental model for the determination of seizure mechanisms. An ideal method would induce seizures by a physical stimulus in freely moving animais. Such seizures would be easily produced, stable and reproducible over time. Based on the fact that seizures occur from widespread, abnormal, high frequency, electrical discharges in the brain, an investigation designed to elicit overt seizures produced by local electrical stimulation of the hippocampus in freely moving animals, was undertaken. This principle has been employed to study seizure initiation and discharge via recorded electroencephalograms (Rosenblueth and Cannon, 1942; Andy and Akert, 1953; 1955; Green and Shimamoto, 1953; Delgado and Mihailovic, 1956; Green and Adey, 1956; Gangloff and Monnier, 1957; Green et al., 1957; MacLean, 1957a; 1957b; Green, 1958; Strobos and Spudis, 1960; B7um et al., 1961; Schallek and Kuehn, 1963). However, overt seizures produced in this manner have seldom been investigated, especially in relation to drugs. Roelofs (1967) is perhaps one of the earliest workers to examine such overt seizures in freely moving cats. He studied the influence of initial behavior on seizure susceptibility by stimulating several areas of the brain via implanted, bipolar electrodes. With some modification, Roelofs's technique was 
adapted in this study to develop overt seizures in unrestrained rats. Electrical stimulation was made through electrodes implanted in the right, dorsal hippocampus. This structure was selected because of its vulnerability to seizures (Kaada, 1951 ; Earle et al., 1953; Andy and Akert, 1955; Green et al., 1957; Green, 1965).

In order to relate this type of seizure to that induced by other experimental procedures, the phenomenon was subjected to the influence of two prototype anticonvulsants, e.g., diphenylhydantoin and trimethadione. These compounds were selected due to their different actions on maximal and minimal seizures as well as their abilities to protect against various kinds of clinical seizures (Millichap, 1965; Toman, 1965; Swinyard, 1969). It was felt that from these data some indication to the electrophysiological mechanisms involved in overt seizures induced by the present method would be obtained. Such deductions would be based on existing knowledge of the action of these drugs on nerve transmission and seizure spread.

Diphenylhydanto in abolishes the tonic phase of maximal (tonicclinic) electroshock seizures by preventing the spread of seizure discharge in the brain (Barany and Stein-Jensen, 1946; Toman et al., 1946; Toman and Goodman, 1948; Espl in and Zablocka, 1969). This action is also seen with maximal seizures produced with picrotoxin or pentylenetetrazol (Goodman et al., 1946, 1953; Toman et al., 1946; Swinyard et al., 1952). Moreover, acute administration of the compound in immature rats reduces the duration of tonic hind 7 imb extension of spinal cord seizures (Vernadakis and Woodbury, 1964). 
Using the developing animal as a model, Vernadakis and Woodbury (1965; 1969) found that diphenylhydanto in increased low frequency electroshock seizure thresholds (1.f. EST) in mice but did not affect the threshold of high frequency 60 cycle, alternating current-induced seizures (a.c. EST). Complication with drug-induced discharge of subcortical excitatory systems e.g., the hypothalamico-adenohypophysealadenocortical system, is suggested as a possible explanation of the a.c. EST response. In the developing animal, diphenylhydantoin al so lowered the convulsive dose 50 (CD50) of picrotoxin and strychnine-induced seizures, indicating that the drug blocks presynaptic and postsynaptic inhibition. When tested against pentylenetetrazol-induced seizures, it lowered thresholds in rats less than 14 days old, but raised CD50 of pentylenetetrazol seizures in older animals, an effect which diminished with age. These workers suggested that the drug probably antagonized presynaptic inhibition to lower pentylenetetrazol seizure threshold. The CD50 of strychnine in strychnine seizures was increased by diphenylhydantoin in 4-day-old rats, presumably due to enhancement of postsynaptic inhibition in the spinal cord, which is mature at this period. In rats between 8 and 12 days of age, diphenylhydantoin lowered CD50 of strychnine. These findings suggest that diphenylhydanto in has 2 actions at the synapse. The net effect of the drug depends on the particular system predominating in the central nervous system at that time.

Diphenylhydantoin also increases the convulsive threshold of the motor cortex in the monkey (Delgado and Mihailovic, 1956). Several 
workers who measured electrical afterdischarges eyoked by stimutation of specific areas in monkeys, cats and rabbits (Delgado and Mihailovic, 1956; Strobos and Spudis, 1960; Schallek and Kuehn, 19.63) general1y observed an increase in threshold and shorter discharge duration in the motor cortex, thalamus and septal areas following drug treatment. However, the compound was also reported to produce no effect on cortical or hippocampal afterdischarge in rabbit (Gangloff and Monnier, 1957). These workers noted some depression of diencephalic excitability but Morrell and co-workers (1959) reported that the drug had no action on spontaneous discharge. Pentylenetetrazol activation of chronic experimental seizure foci in the visual cortex of the rabbit was found to be unaffected. In addition, diphenylhydantoin did not prevent diencephalic projection of the seizure focus. It was, however, very effective in blocking cortical spread of the seizure activity from the focus. In Woodbury's investigation (1955), diphenylhydantoin decreased intracellular concentration of sodium ion in brain cells. Further, it prevented intracellular increase of the same ion in hypoatremic animals, while elevating seizure threshold towards normal. The intracellular increase in sodium caused by maximal electroshock seizures was also prevented when the drug inhibited the tonic phase of the seizure. These observations indicate that the action of diphenylhydantoin to prevent seizure spread is related to an improvement of the extrusion of sodium ions from brain cells, probably by an enhancement of the membrane ion pump. This proposal is supported by the fact that diphenylhydantoin antagonizes many of the effects on skeletal and cardiac 
muscles produced by cardiac glycosides (Woodbury, 1963, Mercer and osborn, 1967), which are known to act primarily by inhibiting the sodium-potassium pump (Judah and Ahmed, 1964; Skou, 1965). Hence, the abilities of diphenylhydantoin to inhibit PTP, tonic hind limb extension (of maximal seizures), spread of epileptic discharge, genesis of grand mal epilepsy and to enhance sodium ion extrusion in cel is indicate that these phenomena are probably interrelated. Thus, if seizures, induced by the present method are modified following diphenylhydantoin administration, their genesis probably involve the above phenomena.

In contrast, trimethadione has very low protective index against maximal electroshock seizures (MES) but is very effective in raising 1.f. EST, a.c. EST as well as electroshock thresholds in hyponatremic, cortisone or thyroxine treated animals (Swinyard et al., 1946; Fingl et a1. , 1952; Woodbury et al., 1952; Swinyard, 1969). In addition, this compound can prevent all the central excitation symptoms produced by pentylenetetrazol in several species (Goodman et al., 1946; 1953; Toman and Goodman, 1948; Toman, 1965). The converse is also true, i.e., pentylenetetrazol is capable of antagonizing central nervous system depression due to large doses of trimethadione. These actions suggest a common site of action of the two agents.

In more detailed studies on the spinal cord (cat), trimethadione, unlike diphenylhydantoin, was found to have no effect on PTP (Esplin and Curto, 1957). Its most significant action (again different from diphenylhydantoin) was to intensify the depression of synaptic transmission, following each transmitted volley of impulses. This effect 
was increased by repetitive stimulation and was accomplished without alteration of isolated impulse transmission. Since pentylenetetrazol had the opposite effect and was characterized as a specific "facilitator" at excitatory synapses, trimethadione was suggested to act as an "anti-facilitator" (Toman, 1965). Its main action may be to selectively block transmission of showers of impulses.

Unlike diphenylhydantoin, trimethadione is effective in raising thresholds of adult animals to seizures induced by picrotoxin or strychnine (Goodman et al., 1946; 1953; Toman and Goodman, 1948).

Schallek and Kuehn (1963) found that trimethadione was superior to diphenylhydantoin in increasing electrical seizure thresholds of cortical and other sites. Furthermore, thresholds to electrical afterdischarges in the motor cortex and thalamus were elevated and the duration of these afterdischarges were increased in the monkey, cat and rabbits, subsequent to treatment with this drug (Delgado and Mihailovic, 1956; Gangloff and Monnier, 1957; Strobos and Spudis, 1960; Schalleck and Kuehn, 1963). In the studies of Morrell et al. (1959), the compound not only suppressed the chronic epileptogenic foci, but also depressed the projection of seizure activity from cortical foci to the thalamus and to the contralateral side. Cortical spread was selectively unaffected. From these findings it is suggested that trimethadione probably alters seizure thresholds by suppressing neuronal discharges which build up a self-oscillating loop in the thalamus. Such a mechanism is probably involved in the precipitation and main- 
tainence of minimal seizures (see GENERAL INTRODUCTION) and to be independent of PTP.

The above discussion indicates that seizure threshold changes subsequent to trimethadione treatment are probably related to the drug's ability to depress transmission of volleys of impulses at the synapse. Any alteration in seizure excitability after i.p. trimethadione in the present study would suggest participation of this mechanism. In addition, genesis of these seizures must also involye the development of a self-oscillating loop in the thalamus.

\section{B. Methods}

Under pentobarbital anesthesia rats were stereotaxically implanted with stainless steel, bipolar electrodes in the right, dorsal hippocampus according to the rat atlas of Konig and Kl ippel (1963; see APPENDIX for detailed procedure). Following surgery, they were housed individually in plastic cages $(16 \times 25 \times 25 \mathrm{~cm}$., Keystone Plastics Co., Media, Penn.). After a recovery period of at least a week, the anima 1 was transferred to a testing chamber $(30 \times 30 \times 50 \mathrm{~cm}$.) in which seizures were induced. This was equipped with an overhead light and a one way mirror. The implanted electrodes were connected to a circuit as illustrated in Figure 1. The stimulus was generated by a Grass stimulator (Model S4, Grass Instrument Co., Quincy, Mass.) in series with a capacitor (Model ACD-13 Decade Capacitor, Aerovox Corp., New Bedford), which maintained a constant waveform. The timer key was set for 6 sec. to control stimulus duration and an external resistor (100 ohm) was placed in series with the electrodes so that current 
Figure 1. Circuit diagram. S, stimulator; C, capacitor;

$E$, electrodes; $T$, timer key; $r, 100$ ohm resistor;

$R$, resistor box; 0 , cathode ray oscilloscope. 


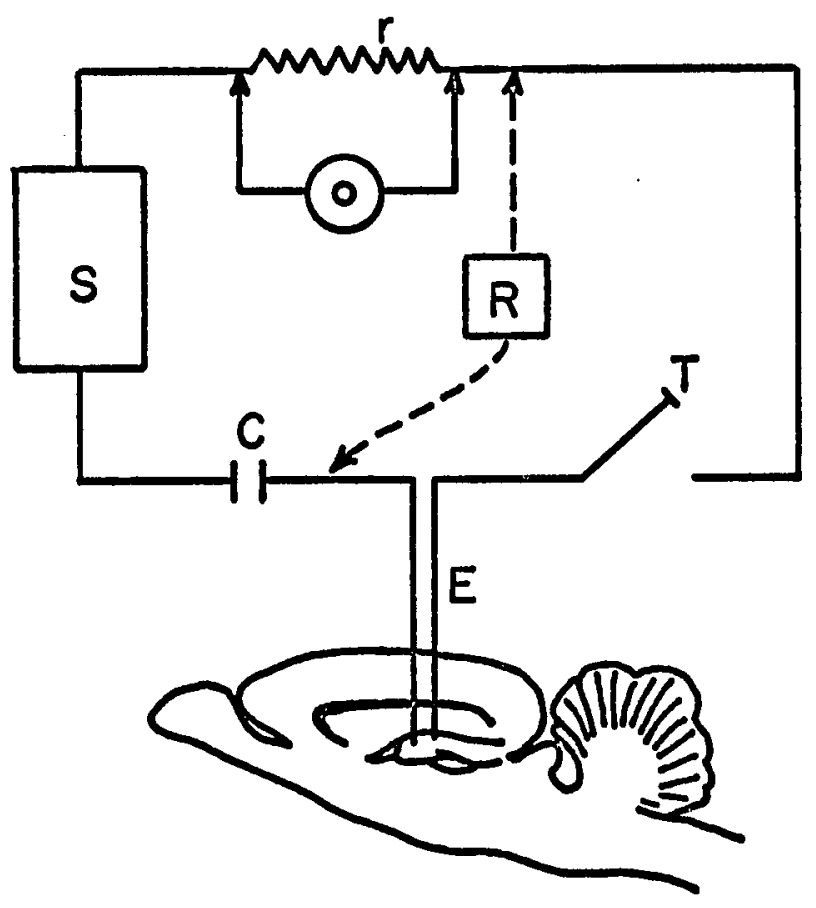


could be measured by a previously calibrated single beam, cathode ray oscilloscope (Type 304-A, Allen B. Dumont Laboratories Inc., Clifton, N.J.). When the key was opened a resistor box (Decade Resistor, Model ARD-41, Aerovox Corp., New Bedford, Mass.) was connected in series with the stimulator to measure rat resistance (electrode impedance).

In this environment, the rat was unrestrained and moved about freely. After 5 min. adaptation it was subjected to a series of electrical stimulations of increasing intensities until a minimal seizure was seen. The stimulus consisted of a train of biphasic pulses ( $6 \mathrm{sec}$. duration, $0.2 \mathrm{msec}$. pulse width) with a frequency of 60 c.p.s. Each stimulus was separated by a $2 \mathrm{~min}$. interval. In order to minimize the total number of stimuli administered to any rat, the following schedule was employed. A starting current (150 uA) was increased in steps of $50 \mathrm{uA}$. At intensities of $600 \mathrm{uA}$ the increment was raised by a factor of 4 and above 1000 uA, it was elevated 10 times the original value. The maximum current administered was 3,000 uA. (In examination of drugs which lowered seizure threshold, the starting current was $100 \mathrm{uA}$ with increments of $25 \mathrm{uA})$.

A seizure was defined as the presence of readily observed facial clonus, rhythmic movements of the jaws and/or clonus of the fore 1 imbs. The current that just produced these symptoms was defined as the seizure threshold. In drug studies, rats which did not exhibit seizures with the maximum current were assigned seizure thresholds of 3,000 uA for statistical computations. 
In deyeloping this method, the intensity of the stimulus was measured as current. According to Ohm's Law, current varies inverseiy as the resistance in a circuit. Since resistance (tissue impedance between the electrodes, called rat resistance in this study) changes with the physiological state of the animal and during a seizure (Roldan et al., 1963; Adey et al., 1966; Elazar et al., 1966; Ranck, 1966) as well as with implantation time (Delgado, 1961), the stability of rat resistance over time was evaluated. Its stability over the experimental life span of the test animal was also recorded. Seizure thresholds of these animals were also examined over the same length of time to provide some indication of the variability of this parameter. In addition, seizure latency and duration were recorded.

The effect of drugs on seizure threshold was examined using a crossover design with a 7 day interval so that each rat seryed as its own control. Groups of 6 to 10 (average of 8 ) rats with pre-determined stable seizure threshold (SST) were employed. Half of these received the drug and the other half, the drug vehicle. Seizure threshold after drug treatment (DST) and control seizure threshold (CST) were determined at the time of peak neurotoxic effect of the drug. Threshold ratios, i.e., DST/SST and CST/SST with $95 \%$ confidence 1 imits were calculated using the method described by Goldstein (1964a).

The rats were eventually sacrificed with pentobarbital $(45 \mathrm{mg} . / \mathrm{kg}$. and perfused with saline, followed with $10 \%$ formal in via the right ventricle. Serial, celloidian sections (30 microns) were stained by the technique of Kluver and Barrera (1953) for microscopic localization 
of electrode tract as well as for evaluation of tissue damage (see APPENDIX for details).

C. Results and Discussion

The data obtained from an evaluation of the stability of rat resistance, presented in TABLE 2 were analyzed by a paired comparison " $t$ " test (Goldstein, 1964b).

TABLE 2. The Stability of Rat Resistance

\begin{tabular}{cccc}
\hline $\begin{array}{c}\text { Min. Between } \\
\text { Test }\end{array}$ & $\begin{array}{c}\text { Mean Resistance } \\
\text { Initial }\end{array}$ & $\begin{array}{c}\text { (Kohm) } \\
\text { Final }\end{array}$ & $\begin{array}{c}\text { Difference Mean } \\
\text { (Kohm) }\end{array}$ \\
\hline 2 & 12.51 & 11.93 & $0.58^{\mathrm{a}}$ \\
15 & 13.05 & 13.62 & $0.57^{\mathrm{a}}$ \\
20 & 12.80 & 12.94 & 0.14 \\
30 & 12.91 & 14.09 & $1.18^{\mathrm{a}}$ \\
1,440 & 13.39 & 13.61 & $0.22^{\mathrm{a}}$ \\
\hline
\end{tabular}

a $\quad P<0.05$

It is evident that over time intervals ranging from 2 to 1,440 min., rat resistance varied significantly in ail but one test. These results emphasize the importance of taking the instantaneous rat resistance into consideration in any measurement of current passing 
through the brain. In all studies rat resistance was measured and the actual current passing through the electrodes was recorded. From the table, rat resistance is seen to vary from 11.93 to $14.09 \mathrm{Kohm}$. Analys is of this parameter over experimental periods of 3 to 12 months indicate a similar trend, with changes in both directions. This does not completely agree with Delgado's observation that tissue impedance increased with implantation time due to possible scar tissue formation in the region around the electrode tips (Delgado, 1961).

The stability of seizure threshold over time is presented in Figure 2. The vertical bars denote $95 \%$ confidence 1 imits and any point where the bar does not cross 1.0 indicates a significant change in seizure threshold. These data show that seizure threshold remained essentially stable over time intervals ranging from 10 to $60 \mathrm{~min}$. between determinations. Additional studies with injection of saline between tests also produced no apparent change in threshold over the same time intervals. Although seizure threshold varied from animal to animal ( 150 to $500 \mathrm{uA}$ ), it remained essentially predictable and constant from day to day. However, with implantation time, there was a tendency towards a reduction of seizure threshold. Since the manifestation of a full minimal (clonic) seizure involves the discharge of a large number of neurons for a considerable time, the reduction in seizure threshold with implantation time seems to indicate a progressive ease of developing the cortico-thalamic oscillating loop with repeated stimulation. This possibility is supported by the rare occurrence ( 2 of the older rats) of continuous seizures (up to $1 \mathrm{hr}$. 
Figure 2. Stability of seizure threshold over time. Threshold Ratio was determined by dividing the final seizure threshold by the initial seizure threshold. The vertical bars denote $95 \%$ confidence 1 imits. 


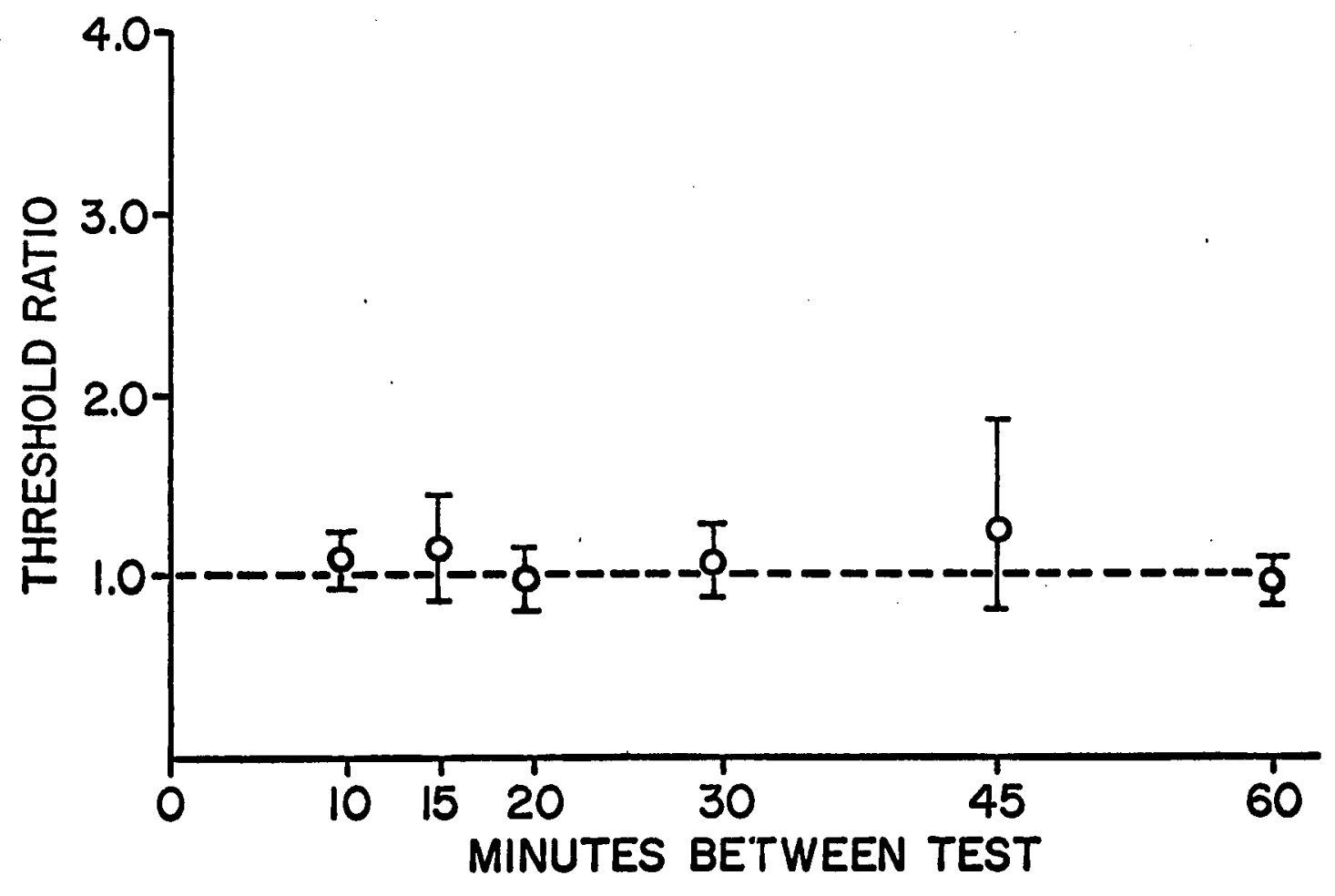


in length, punctuated by post-ictal depression), subsequent to a previously subthreshold current.

of the 73 animals implanted with electrodes in this study, only 1 failed to produce seizures after 5 stimulation sessions. Examination of this animal indicated that the 2 parts of the electrodes were in poor contact with each other. In addition, 2 animals pulled out their electrodes and 2 exhibited seizures merely on being placed in the experimental box. This observation is interesting and reveals the possibility of conditioning rats to such seizures. Both unconditioned and conditioned stimuli have been employed by investigators to induce seizures. Dol in (1939) conditioned dogs to seizures by associating the interoceptive stimulus with intragastric administration of convulsant drug (camphor 0i1). Ungher et a1. (1952) were able to produce seizures with exteroceptive (optic, olfactory or acoustic) and interoceptive (intragastric pressure) stimuli by associating them with the intragastric administration of camphor oil. These workers also associated camphor oil administration with the laboratory environment of the animal and successfully produced conditioned seizures in this manner. An electroshock seizure was reported to be produced on exposure to the laboratory environment in which the animal was subjected to the shocks. Even the sounds produced by the click of the electroshock apparatus can condition an animal to seizures (Kreinder, 1965)! It was reported by this author that in one study, a dog exhibited the signs of a minimal seizure immediately on application of the electrodes to the head without any passing current. Previously the dog had been 
subjected to 19 convulsive seizures in the same environment. This observation is very similar to the situation seen in this study.

The latency of seizures at threshold current varied from 1.5 to $50 \mathrm{sec}$. between animals, was constant for some rats but variable for others over experimental periods of up to 12 months. The duration of these seizures (untreated animals) varied from $5 \mathrm{sec}$. to $7 \mathrm{~min}$. with 3.5 min. being the usual length of a seizure (the end of the seizure was indicated when the rat suddenly resumed exploratory or grooming behavior).

The lesion produced by electrode implantation was reconstructed with the aid of the rat atlas of Konig and Klippel (1963) and is represented in Plate I. Minimal extent of the brain lesion refers to the brain area where damage occurred in 25 to $100 \%$ of the experimental rats, while maximal extent of the lesion represents the brain area where lesion was seen in less than $25 \%$ of the test animals. Representative frontal sections (A-D) at the stated distances (in microns) anterior to frontal or ear-bar zero indicate the size of the lesion in the anterior-posterior direction. In these diagrams the abscissa represents distance in $\mathrm{mm}$. from saggital zero, while the ordinate denotes length in $\mathrm{mm}$. above or below ear-bar zero. A representative saggital section at 1,490 microns (u) lateral (to the right) from the mid-line is presented in $E$. The abscissa denotes distance (in mmi) anterior or posterior to ear-bar zero while the ordinate indicates the distance (in mm.) above or below ear-bar zero. As shown in Plate I, tissue damage extended from about $2.1 \mathrm{~mm}$. to $4.5 \mathrm{~mm}$. anterior to frontal 
Plate I. Minimal () and maximal ( ) extent of the brain lesions projected on frontal sections (A-D) and saggital section (E) at the stated distances from frontal and saggital zeros, respectively. NC, neocortex; CC, corpus callosum; H. hippocampus . 

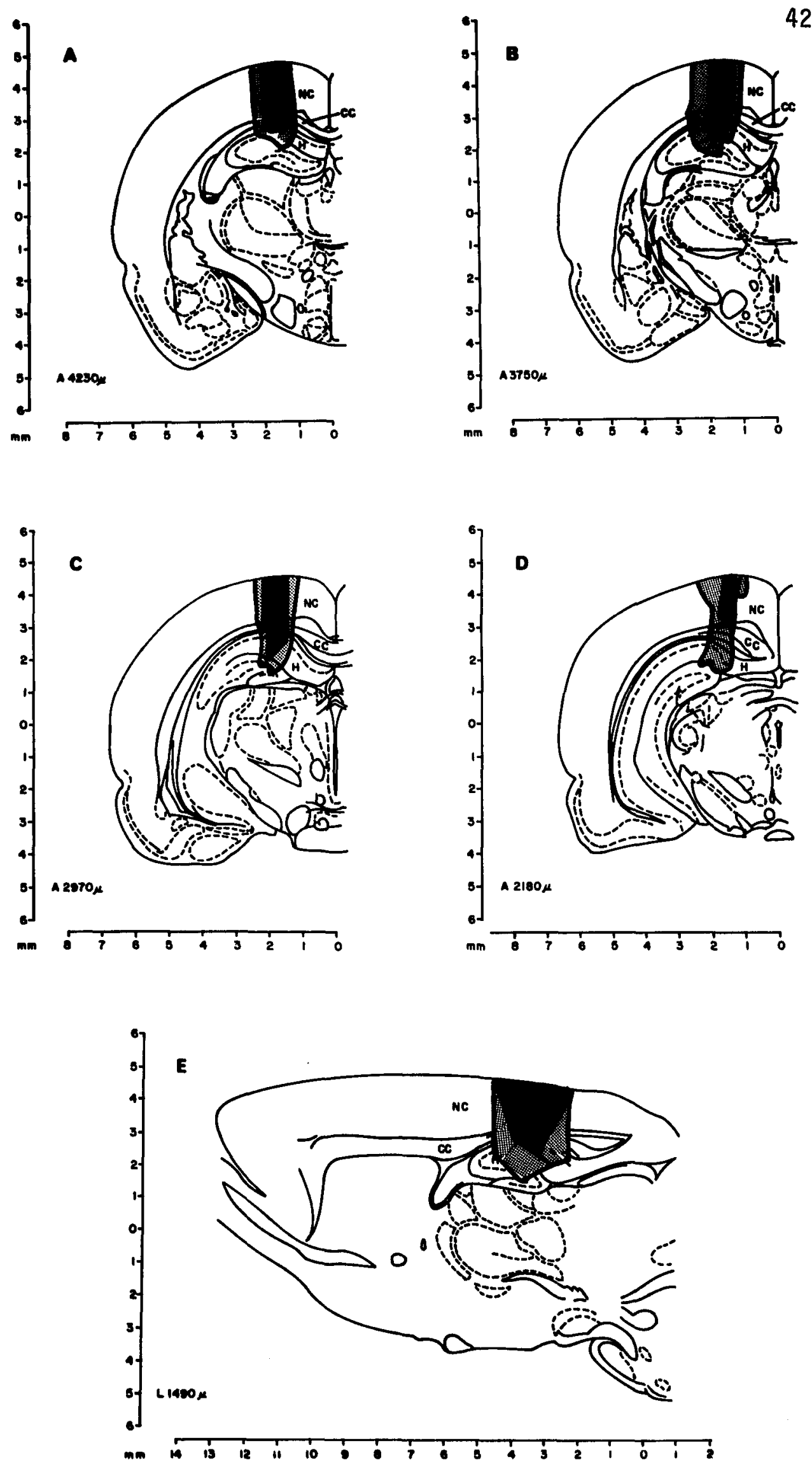
zero and from $1.1 \mathrm{~mm}$ to $2.8 \mathrm{~mm}$. lateral to saggital zero (on the animal's right side). Only the neocortex (NC), the corpus callosum (CC) and the dorsal hippocampus (H) in this region were affected. In all the animals studied, the electrode tip lay in the right, dorsal hippocampus between 3.7 and $3.8 \mathrm{~mm}$. anterior to frontal zero. The lateral location of the electrode tip was 1.1 to $2.0 \mathrm{~mm}$. lateral to saggital zero as measured using the mid-line of the cerebrum as a reference. This was different from the intended site which was $2.59 \mathrm{~mm}$. lateral to saggital zero. In determining the lateral co-ordinate for the electrode site during implantation, the mid-line of the skull was taken as saggital zero. The histological studies, therefore, indicate that the mid-line of the skull does not correspond exactly with the mid-1 ine of the cerebrum in the rat. However, this discrepancy is relatively unimportant in the present experimental design, since the hippocampal sites stimulated in the rats did not differ by more than $0.5 \mathrm{~mm}$. in any of the experimental animals. From the above observation the discrepancy between the mid-line of the skull and the cerebrum was about $1 \mathrm{~mm}$. in the rats used in this study. The size of the lesion in each animal varied from 0.4-0.7 mm. (anterior-posteriorly) and from 0.4-0.7 mm. (1aterally). Except for damage due to the electrode wires, there was little injury due to tissue reaction to the metal. Thus, the lesion made by the electrodes was minimal and did not include any brain formations except those in the electrode tract.

The effect of diphenylhydantoin on seizure threshold is demonstrated in Figure 3. Control solution did not alter seizure threshold signifi- 
Figure 3. Effect of diphenylhydantoin on seizure threshold. Threshold Ratio was determined by dividing the drug seizure threshold or the control seizure threshold by the stable seizure threshold. The vertical bars denote $95 \%$ confidence 1 imits. $\Delta$, diphenylhydantoin; $O, 10 \%$ propylene glycol in saline. 


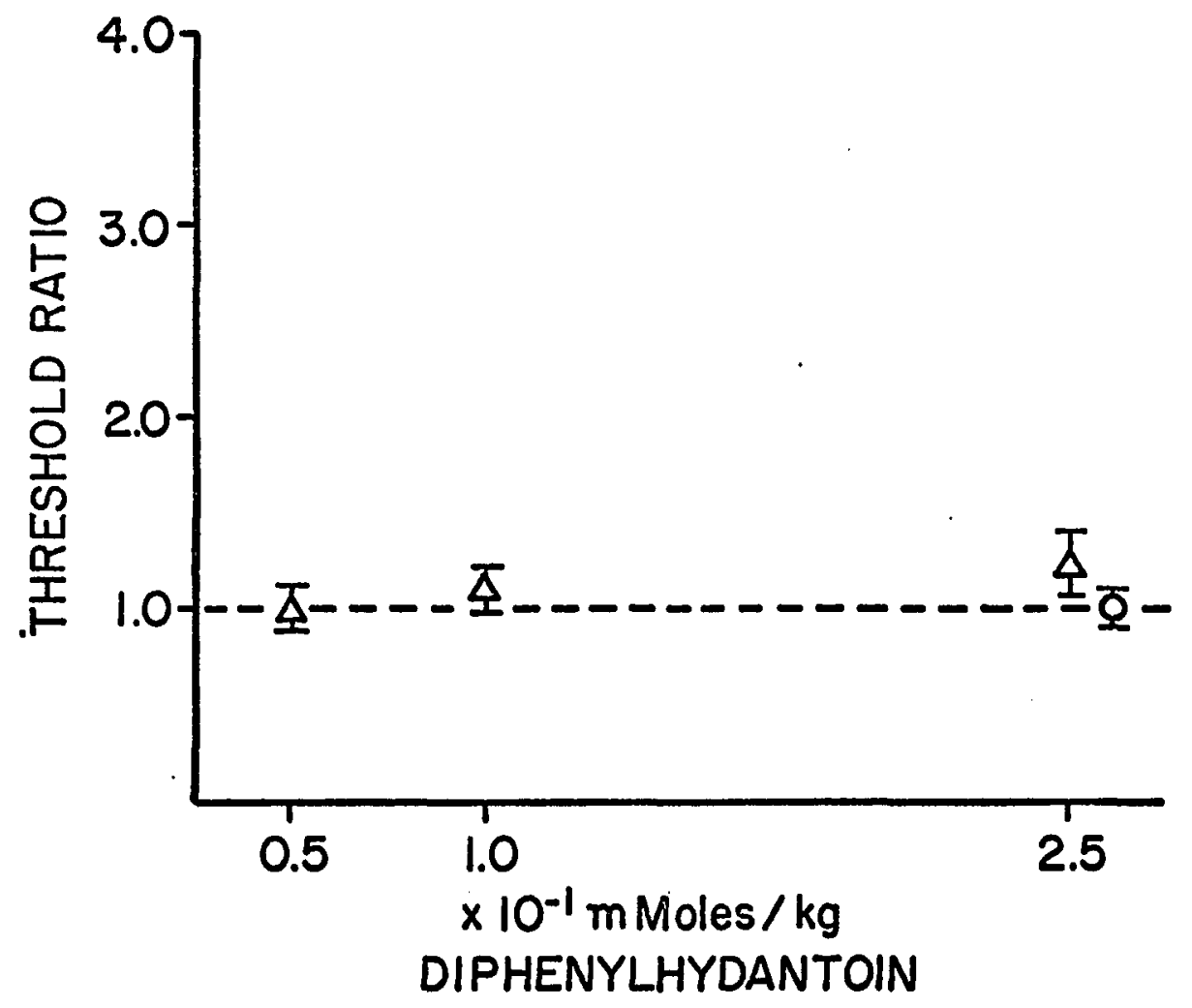


cantly in this or subsequent studies. At doses of 0.5 and $1.0 \times 10^{-1}$ mMoles/kg., this compound did not have a significant effect, while a higher dose of $2.5 \times 10^{-1} \mathrm{mMoles} / \mathrm{kg}$. produced a slight, but significant elevation of threshold. These results agree with those reported for diphenylhydantoin with other experimental models of minimal seizures, in that low doses have no effect on threshold to metrazol seizures, a.c.EST or 1.f.EST, while higher doses increase 1.f.EST (Swinyard et al., 1952; Brown et al., 1953; Wolf et al., 1962; Swinyard, 1969; Vernadakis and Woodbury, 1969). Since the susceptibility of these rats to minimal seizures did not change with diphenylhydantoin except at high doses, it is reasonable to suggest that the genesis of such seizures does not involve the phenomenon of PTP. The slight elevation of threshold seen with the high dose of diphenylhydantoin may be related to the non-specific stabilizing action of this drug on excitable membranes (Toman, 1952). This could result from the more efficient extrusion of sodium ions from brain cells, probably by stimulation of the metabolic sodium pump (Woodbury, 1955; Toman, 1965; Espl in and Zablocka, 1969).

The effect of trimethadione on the susceptibility of animals to seizures induced by stimulation of the hippocampus is presented in Figure 4. All 4 doses raised threshold significantly and an apparent dose response relationship was seen. The efficacy of trimethadione in this procedure was further emphasized by the absence of seizures in several animals at the maximum stimulus employed. Again, these data reflect anticipated results, in that trimethadione is well known to elevate minimal seizure threshold as measured by other techniques 
Figure 4. Effect of trimethadione on seizure threshold. Threshold Ratio was determined by dividing the drug seizure threshold or the control seizure threshold by the stable seizure threshold. The vertical bars denote $95 \%$ confidence 1 imits. At doses higher than $5.0 \times 10^{-1} \mathrm{mMoles} / \mathrm{kg}$., several animals did not exhibit seizures with the maximum current and were assigned seizure thresholds of $3,000 \mathrm{uA}$ for statistical computations. $\triangle$, trimethadione; 0 , saline. 


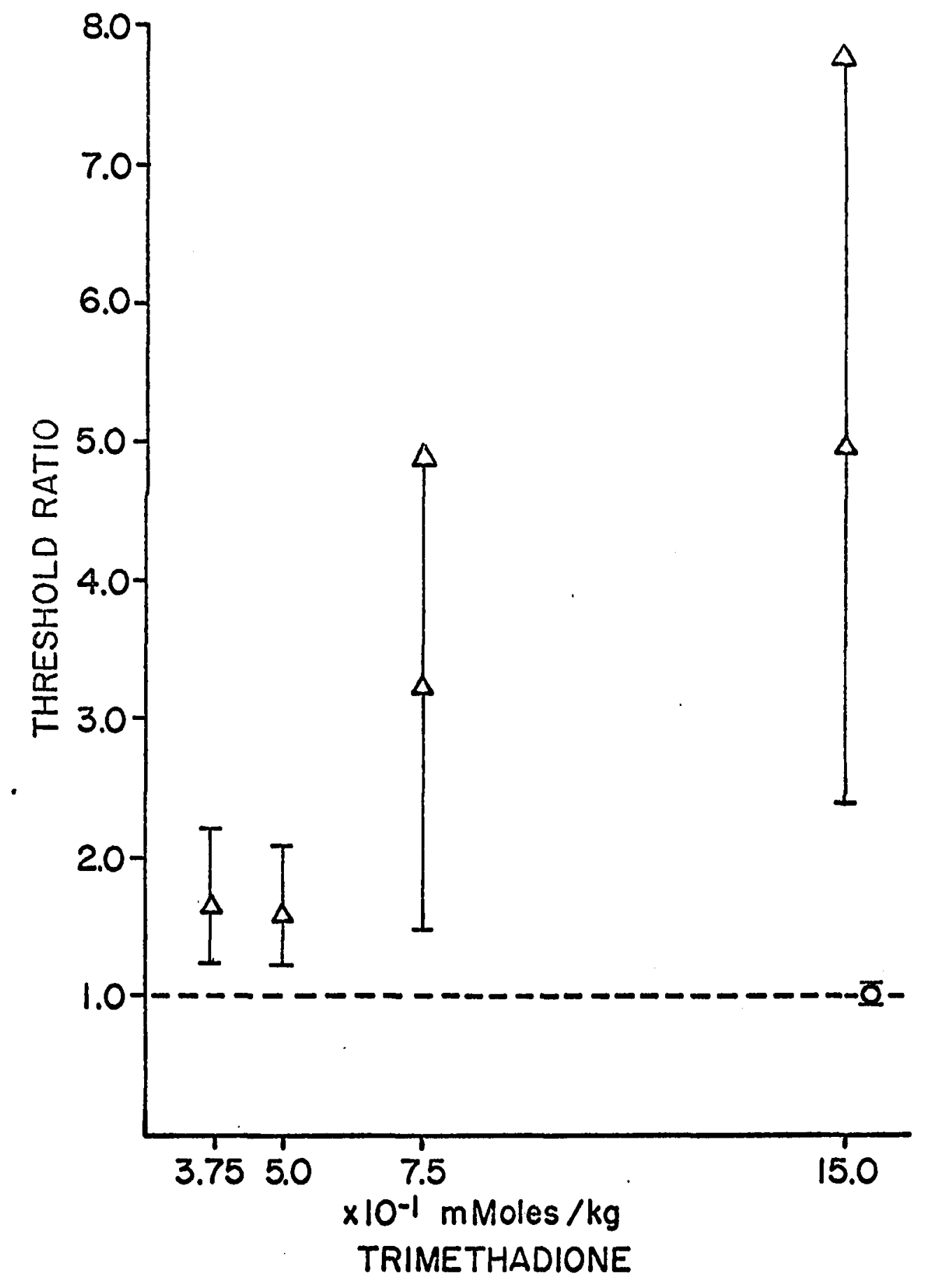


(Șwinyard et al., 1952; Brown et al., 1953; Toman, 1965; Swinyard, 1969).

As indicated earlier, trimethadione suppresses chronic epileptogenic foci and depresses the projection of seizure activity from the cortex to the thalamus, probably via its ability to deepen depression at the synapse following transmission of an impulse. This probably prevents the build-up of a self-oscillating loop in the thalamus which is probably responsible for the manifestation of the seizure. The seizure produced must also involve the spread of discharge to both sides of the brain since movements of both sides of the fore 7 imbs were generally observed. The ability of trimethadione to suppress contralateral spread of abnormal electrical activity (see introductory part of this section) may therefore, contribute to its anticonvulsant effect.

The data obtained in this study indicate that reproducible minimal seizures can be induced by electrical stimulation of the hippocampus in unrestrained rats. Further, susceptibility to such seizures is readily reduced by trimethadione and not by diphenylhydantoin. By comparing these observations with the known actions of these two prototype anticonvulsant agents on neuronal transmission and seizure spread, it is reasonable to suggest that seizures induced by hippocampal stimulation probably result from excitation of a group of neurons discharging between the thalamus and the cortex, a mechanism which probably does not involye PTP. 
The aboye explanations of diphenylhydanto in and trimethadione actions are speculative and based on the ability of these drugs to alter electrical afterdischarges and transmission at spinal cord neurons as reported by other workers. It is likely that the influence of these drugs on seizure susceptibility may have resulted through other actions, e.g., influence on hormones, distribution of electrolytes and water, blood pH and central metabolism (Woodbury, 1969). At present, diphenylhydantoin is known to influence the release of pituitary hormones. The drug stimulates the release of thyroid stimulating hormones (TSH) at low doses while it depresses TSH release at high doses (Woodbury, 1969). Since these changes influenced nervous system excitability, it can therefore effect seizure susceptibility. Further, diphenylhydantoin stimulates sodium ion extrusion in brain cells and tends to stabilize excitable membranes (see Introduction). Thus, changes of seizure thresholds after these drugs could have resulted from the actions of these drugs on other factors besides electrophysiological al terations. In order to support and confirm the suggestion that minimal seizures produced in this experiment involved the existence of electrical discharges which formed a recurrent self-oscillating cortic-thalamic loop, recording electrodes should be implanted at various cortical and subcortical sites. EEG should be monitored during and following electrical stimulation to show the electrical events occurring during the genesis of a seizure. By comparison of data from control studies with those obtained in drug studies, information with regard to how diphenylhydanto in and trimethadione act electrophysiologically to alter seizure threshold in this experiment should be provided. 
In conclusion, the data obtained in this study with the 2 prototype anticonvulsant agents indicate that seizures induced by electrical stimulation of the hippocampus resemble minimal seizures induced by other experimental techniques. 
IV. THE IMPORTANCE OF ADRENERGIC TONE TO SEIZURES INDUCED BY ELECTRICAL STIMULATION OF THE HIPPOCAMPUS.

\section{A. Introduction}

In the general introduction evidence for the involvement of acetylcholine, serotonin, gamma-amino-butyric-acid and other neurotransmitters in seizure behavior was presented. In this section the importance of catecholamines, especially norepinephrine, will be examined.

The catecholamines thought to be involved in seizure expression are norepinephrine and dopamine. These two amines as well as epinephrine are distributed throughout the mammalian nervous system in a non-uniform manner. Whereas epinephrine and norepinephrine predominate in the hypothalamus, the highest amount of dopamine occurs in the caudate nucleus, putamen regions, the substantia nigra and the globus pallidus (Vogt, 1954; Montagu, 1957; Weil-Malherbe and Bone, 1957; Carlsson et al., 1958; Bertler and Rosengren, 1959; Carlsson, 1959; Sano et al., 1959; Bertler, 1961; Hornykiewicz, 1966).

The development of specific and sensitive histochemical methods for the determination of norepinephrine, epinephrine and dopamine by Swedish investigators (Falck, 1962; Falck et al., 1962; Corrodi and Hillarp, 1963; 1964) has made possible the detail mapping of these neurones in the mammalian brain and spinal cord. The available infor- 
mation on this subject is adequately reviewed by Hillarp et al., 1966. Both norepinephrine and dopamine nerve cells have similar general characteristics, with low concentrations of amines in the perikaryon and none in the nucleus. Most of these nerve cells are in the mesencephalon, where they can be divided into 3 groups; a large group present in the ventrolateral part of the reticular formation; a large group present in the substantia nigra (mainly in the zona compacta) and a very large group surrounding the cranial half of the nucleus interpeduncularis. According to the classification of Dahlstrom and Fuxe (1964) for central aminergic neurons, these groups fall into $A 8, A 9$ and $A 10$, respectively. When a lesion is made at the caudal part of the inferior colliculi, it interrupts all ascending catecholamine fibers from the pons and medulla oblongata, but leaves intact, areas of the brain that contain high dopamine nerve terminals (e.g., nucleus caudatus putamen, nucleus accumbens and tuberculum olfactorium; Fuxe et al., 1964; Anden et al., 1965a; 1965b; Fuxe $1965 \mathrm{a} ; 1965 \mathrm{~b})$. Such a lesion decreases norepinephrine nerve terminals in areas cranial to the lesion. These results are supported by biochemical data which shows that norepinephrine, but not dopamine levels are considerably decreased in brain regions cranial to the lesion. These data indicate that dopamine nerve cells are present almost exclusively in the mesencephalon, with most of them in group A9. This cell group has been postulated to be the main nucleus of a large unilateral nigro-neostriatal dopamine neuron system (Aden et al., $1965 \mathrm{a} ; 1965 \mathrm{~b})$. Dopamine neurons are also present in A10, but not in 
A8 (Hillarp et al., 1966). Experiments involving selective destruction of the median eminence (where dopamine terminals are present) demonstrated the presence of a tubero-infundibular dopamine neuron system (Fuxe, 1964; Hillarp et al., 1966). The dopamine cells give rise to very fine varicose terminals ( 0.3 to 0.6 micron). They are densely packed in all the dopamine areas with a concentration about 300 times that of the perikaryon.

Most of the norepinephrine cell bodies probably exist in the pons and the medulla oblongata. These have been divided into 7 groups according to the classification of Dah1strom and Fuxe, 1964. The largest groups in the medulla oblongata are medium sized group in the ventrolateral part of the reticular formation (A1), a sma11 group (A2) in the nucleus commissuralis, a few small cells in the nucleus ovlivaris accessorius dorsalis (A3) and a small group in the lateral part of the roof of the fourth ventricle at the level of maximum development of the facial nucleus (A4). In the pons, two small groups are present within the rubro-spinal tract (A5). A large group is also located in the nucleus coeruleus (A6), while a small number of cells can be found within the reticular formation (A7), ventral to the ventral part of the superior cerebellar peduncle. Like the dopamine nerve cells, the norepinephrine neurons have a zone around the nucleus which contain higher concentrations of amines. The nerve cells of groups $A 1$ and $A 2$ send fibers down into the anterior and lateral funiculi. These give rise to most of the norepinephrine nerve terminals in the gray matter of the spinal cord (Carlsson et al., 
1964; Dahlstrom and Fuxe, 1964; Dahlstrom and Fuxe, 1965a). These fibers descend along the spinal cord for various distances, many as far as the lumbrosacral part. These axons give off collaterals to the gray matter along the way. There exist at least 2 different bulbospinal norepinephrine systems. One system travels down in the lateral funiculus, mainly in the dorsal part and innervates the sympathetic lateral column and the posterior horn. The other system innervates the anterior horn (e.g., the lateral motor cell area). About 50\% of the terminals to the sympathetic lateral column cross at the spinal level (via the posterior gray commissure). In the medulla oblongata, half of the fibers of group Al cross over to the other side.

The majority of norepinephrine terminals make axodendritic contacts with interneurons (e.g., in the substantia gelatinosa) or the motor neurons or both. Descending catecholamine nerve fibers are also found in the white matter of the spinal cord. These leave via the ventral thoracic roots without relays on motor neurons or interneurons (Dahlstrom and Fuxe, 1965b). Experiments, employing unilateral lesion on the border of the pons and the mesencephaton, revealed that $70 \%$ of norepinephrine in the prosencephalon probably originates from cells in the pons and medulla oblongata.

In group Al, two different types of neurons are seen: those with ascending fibers and those with descending fibers. The ascending fibers travel in the dorsal part of the reticular formation. Together with norepinephrine fibers originating in the pons, they form a lemniscus in the ventrolateral part of the mesencephalon (region of the 
reticular formation). This lemniscus extends in a dorsal-ventral direction. Most of these fibers pass into the median forebrain bundle which gives rise to norepinephrine terminals in the hypothalamus, the preoptic area, the ventral part of the nucleus stria terminalis, the septal area, the cingulate gyrus, the neocortex, the amygdaloid cortex, the dentate gyrus, the hippocampal gyrus and the parts of the thalamus (Dahistrom et al., 1964; Anden et al., 1965a; Hi1larp et al., 1966). These pathways are mostly uncrossed. In the prosencephation, the norepinephrine terminals are located mainly in the hypothalamus, the 1 imbic lobe and the neocortex. In the lower brain stem, the visceral afferent and efferent nuclei of the cranial nerves have high concentrations of norepinephrine terminals. This is also true of parts of the reticular formation.

The norepinephrine terminals can be very fine ( 0.5 micron) in the neocortex to fairly thick (up to 2 microns) in the hypothalamus (Fuxe, 1965a).

From the above, it is evident that separate norepinephrine and dopamine containing neural pathways exist. Both these neurotransmitters are currently implicated in seizure expression as discussed below.

In 1950 Weil-Malherbe postulated, on the basis of the similarity in psychological effects of glutamic acid and amphetamine, that glutamic acid alleviated certain convulsive disorders through an adrenergic mechanism. Twenty years and hundreds of studies later, the precise involvement of catecholamines in seizure expression is still unre- 
solved. Some workers have postulated an involvement of dopamine, others believed norepinephrine was responsible, while still other investigators held that serotonin was wholely responsible for the varying sensitiveness of animals to seizures.

Some of the support for norepinephrine implication comes from studies which examined brain contents of endogenous norepinephrine in animals with differing susceptibilities to audiogenic seizures. The degree of responsiveness of an animal to sound-induced seizures is genetically determined (Vicari, 1951; Ginsburg and Miller, 1963; Schlesinger et al., 1966; Fuller and Sjursen, 1967). Several workers hypothesized that a lack of gamma-amino-butyric-acid produced animals that were more susceptible to audiogenic seizures (Lyons et al., 1958; Coleman, 1960; Schlesinger et al., 1968a). Other investigators found good correlation between brain amine levels and susceptibility to seizures (Schlesinger et al., 1965; Scudder et al., 1966). For example, strains of audiogenic seizure susceptible mice at the optimal age of susceptibility, have lower norepinephrine and serotonin levels compared to a resistant mouse strain. Further, mouse strains with lower levels of catecholamines and serotonin exhibit shorter latencies to electroshock seizures. However, a recent study by McGeer et al. (1969), refuted this correlation. These workers investigated audiogenic susceptible and unsusceptible rats and mice where the susceptible and the resistant animals were of the same strain. They exposed these animals to 6 audiogenic stimuli on alternate days and sacrificed their animals between 3 to 7 days subsequent to the stimuli. No sig- 
nificant difference between susceptible and unsusceptible animals in norepinephrine or dopamine was seen. Analysis of catecholamines in discrete areas of the brain also showed no significant difference in catecholamine levels between the two test groups. These workers used the same strain of animals as test and control mice, while Schlesinger et al. (1965) compared susceptible strains of mice against a strain resistant to audiogenic seizures. McGeer and his co-workers subjected their experimental animals to the sound stimuli prior to decapitation. One possible factor that may have contributed to the difference in the results seen with the McGeer study is the fact that these workers sacrificed their animals usually more than 7 days, subsequent to the last exposure to the stimulus (employed to establish seizure responsiveness). In the same strain of mice, Schlesinger's group found a fall of seizure susceptibility from 87 to $13 \%$ for tonic seizures, 87 to $40 \%$ for clonic seizures and 100 to $87 \%$ for "wild running" phase of the seizure with a time lapse of 7 days!

The influence of catecholamines in seizure expression has also been investigated through the use of exogenously administered epinephrine and norepinephrine. Most of the findings indicate that catecholamines reduce the susceptibility of animals to seizures. For example, peripheral injections of epinephrine reduce the incidence and lethality of seizures (Mercier, 1949; Busquet and Vischniac, 1950). Other sympathomimetic agents (e.g., extract of genet, ephedrine and amphetamine) examined in the same studies also reduce seizure severity. Swinyard 
et al. (1964) demonstrated that intravenous injection of either norepinephrine or epinephrine elevated thresholds to pentylenetetrazol convulsions, al though higher doses which produced marked circulatory and metabolic disturbances had the opposite effect. In the same study, epinephrine also raised the threshold to electrically-induced seizures. Norepinephrine was ineffective.

Using the intraventricular route of injection to avoid the bloodbrain-barrier for catecholamines, Felberg and Sherwood (1954a) found that norepinephrine and epinephrine antagonized convulsions induced by curare. The "seizure severity scores" of audiogenic seizure susceptible mice were likewise reduced by intracisternal injection of norepinephrine (Schlesinger et al., 1969). In addition, these workers found that the "seizure severity scores" for pentylenetetrazol seizures in these animals were lowered by norepinephrine and serotonin administered intracisternally.

However, there are reports also in the literature which indicate that catecholamines aggravate seizures. Notkin and Pike (1931) reported that intravenous epinephrine increased the severity of absinthe fits in cats. In a similar study, Keith (1933) observed that intravenous epinephrine given 2 to 3 minutes before thujone (constituent of the volatile oil of absinthe) enhanced the severity of convulsions. The refractoriness which followed successive doses of thujone was also el iminated after epinephrine injection. Hall (1938) saw enhanced pentylenetetrazol seizures in rabbits following intravenous epinephrine. This was confirmed by Jasper and Erickson (1941) who 
attributed the effect to a peripheral circulatory action, probably resulting in an increase of pentylenetetrazol flowing to the brain. Epinephrine was reported by Minz et al. (1951) to prolong electricallyinduced seizures in rabbits. Seizure duration in thyroidectomized rabbits is also increased by epinephrine (Albe-Fessard et al., 1951). In tracheotomized cats, injection of epinephrine during supramaximal electroshock markedly prolongs its duration (Ruf, 1951). Similar findings were reported by Minz and Domino (1953) who investigated the influence of intravenous and intraarterial injections of 1-epinephrine and norepinephrine on seizure duration in spinal cats. They examined cortical afterdischarges initiated by supramaximal stimulation in the motor cortex and found that epinephrine and norepinephrine increased seizure duration. By themselves, these drugs did not change the normal electroencephalogram. With repeated injections, both compounds potentiated cortical afterdischarge and shortened cortical extinction time following repeated stimulations, making the cortex 3 times more excitable than norma1. Seizure duration was increased up to 8 times. These workers suggested that the catecholamines enhanced seizure discharge via depression of certain inhibitory cerebral circuits involved in the maintenance of the central excitable state. It is more likely, however, that these effects are related to an increase of available glucose to the brain as a result of activation of phosphorylase (Innes and Nickerson, 1965), since in the same study, high concentrations of glucose prolonged the duration of cortical afterdischarge. 
Peripheral administration of catecholamines in the study of their effects on seizure susceptibility is always subject to the problem of poor penetration from the blood to the brain substance (Draskoci et al., 1960; Marley, 1966). In order to avoid this, investigators have employed several pharmacological methods to alter the endogenous catecholamine levels. The effects of such manipulations on seizure susceptibility were then studied.

The synthesis and catabolism of catecholamines are well established. In vivo, the synthes is of norepinephrine involves the hydroxylation of phenylalanine to tyrosine. The enzyme, tyrosine hydroxylase catalyzes the hydroxylation of tyrosine to dopa. This is the rate limiting step in the total reaction. Through the activity of dopa decarboxylase, dopa is converted to dopamine which in turn is hydroxylated on the beta position to yield norepinephrine (Koelle, 1965; Goldstein, 1966; Udenfriend, 1966; Sourkes, 1966).

Within the nerve terminal, norepinephrine is partitioned into a cytoplasmic mobile pool and intragranular pools (mobile and storage pools) through equilibria between active transport, passive diffusion, synthesis and catabolism via monoamine oxidase. Following release of norepinephrine and its action on effector cells, excess is removed by diffusion, metabolism via catechol-o-methyltransferase (COMT) or by uptake back into the axonal terminals or into extragranular pools (Bloom and Giarman, 1968; Euler, 1968; Anden et al., 1969). Many substances which modify the synthesis and metabolism of catecholamines are available today. Such compounds have been employed in the study of seizure behavior. 
Reserpine which blocks the active transport mechanism for the storage of amines in the granules produces depletion of catecholamines as well as serotonin (Holtzbauer and Vogt, 1956; Paasonen and Vogt, 1956; Pletscher et al., 1956; Carlsson, 1964; Dahlstrom and Fuxe, 1964; Dahlstrom et al., 1965; Glowinski et al., 1966; Shore, 1966). Several workers reported that reserpine lowered seizure threshold and increased the incidence of death following seizures (Chen et al., 1954; 1968a; 1968b; Jenny, 1954; Bielec, 1959; DeSchaepdryver et al., 1962). Cooke and co-workers (1960) observed a shorter latency to seizures after reserpine treatment. In a later study, Cooke (1961) was able to induce audiogenic seizures in unsusceptible rats after reserpine administration. This compound facilitates the occurrence of pentylenetetrazol seizures (Chen and Bohner, 1961; Pfeifer and Glambos, 1967; Jones and Roberts, 1968) and reduces the survivar time of animals given metrazol (Lessin and Parkes, 1959). Lethality following meta-fluorotyrosine-induced seizures is also increased by the drug (Weissman and Koe, 1967).

By treatment with monoamine oxidase inhibitors (e.g., iproniazid or JB-516), intraneuronal metabol ism of catecholamines is prevented (Kopin, 1964). Both iproniazid and JB-516 prevent decrease of seizure threshold by reserpine (DeSchaedryver et al., 1962; Chen et al., 1968a; 1968b). Iproniazid al so antagonizes reserpine-induced reduction of survival time (Lessin and Parkes, 1959) in animals subjected to metrazol seizures. Reserpine facilitation of such seizures is antagonized by intraventricular injections of norepinephrine and 
dopamine (Jones and Roberts, 1968). In this study, norepinephrine returned the ED50 of metrazol to the normal value, whereas dopamine was less effective.

Tetrabenazine, a synthetic compound which acts like reserpine also produces depletion of catecholamines by interference with the storage mechanism of the intraneuronal granules (P1etscher, 1957a; 1967b; Pletscher et al., 1958). This compound reduced survival time of mice to metrazol seizures (Lessin and Parkes, 1959). Iproniazid antagonized this effect. Thresholds to electrically-induced extensor seizures were reduced by about $40 \%$ following tetrabenazine, while in animals previously administered iproniazid this reduction was prevented.

Both reserpine and tetrabenazine produce nonspecific depletion of cerebral amines, since the storage mechanisms of serotonin are also impaired. As a result, the data obtained using these two compounds are not conclusive with reference to the action of catecholamines.

The concentration of endogenous norepinephrine may also be lowered through interference with its synthesis. This is achieved by the use of inhibitors of the different enzymes which catalyze each reaction in the formation of norepinephrine from tyrosine. Thus, compounds which block tyrosine hydroxylase, dopa decarboxylase or dopamine- $\beta$-oxidase may be employed. This principle has been used in evaluating the influence of norepinephrine on seizure susceptibility. 
Dopa decarboxylase inhibitors (e.g., alpha-methyl-m-tyrosine [ $\alpha-m m T]$ and alpha-methyl-DOPA $[\alpha-m D o p a])$, act selectively to lower endogenous dopamine and norepinephrine levels (Hess et al., 1961; Porter et al., 1961). Four hours following injection of $\alpha-m m T$, both catecholamines are reduced, but at $24 \mathrm{hr}$. dopamine level returns to normal, while norepinephrine concentration stays depressed (Carlsson, 1964; Weissman and Koe, 1965). This compound increases the susceptibility of mice to low frequency electroshock seizures (Yeoh, 1966). Maximal electroshock seizures in mice are also enhanced after $\alpha$-mmT treatment (Rudzik and Mennear, 1966a). Chen and co-workers (1968a) however, failed to see any effect with $\alpha$-mmT on threshold to electrically-induced extensor seizures, but observed that it increased seizure thresholds in animals pretreated with iproniazid.

Employing $\alpha$-mDopa, DeSchaepdryver et al. (1962), reported good correlation between lowering of electroshock threshold and reduction of norepinephrine. Other workers saw no effect on thresholds to electrically-induced extensor seizures (Chen et al., 1968a) or susceptibility to metrazol seizures (Pfeifer and Galambos, 1965) after $\alpha$-mDopa. A slight depression of threshold was noticed in animals pretreated with iproniazid (Chen et al., 1968a). In the same study, where seizure threshold was initially elevated with diphenylhydantoin or metazolamide, $\alpha$-mDopa enhanced diphenylhydantoin effect, but did not alter metazolamide action. On the contrary, $\alpha-m m T$ augmented diphenylhydantoin action when administered before the anticonvulsant but antagonized it when given after diphenylhydantoin. Alpha-methyl- 
DOPA opposed reserpine-induced facilitation of metrazol seizures in mice and rats (Pfeifer and Galambos, 1965). These workers reported no lowering of brain norepinephrine when $\alpha$-mDopa was administered in combination with reserpine even though norepinephrine concentration in the brain was reduced in reserpinized animals. Moreover, the anticonvulsant activity of acetazolamide was restored by $\alpha$-mDopa in reserpinized animals (Rudzik and Mennear, 1966a). Such findings are consistent with the hypothes is that norepinephrine suppresses seizure expression. However, $\alpha$-mDopa per se, did not alter susceptibility to metrazol seizures, although in such animals brain norepinephrine was significantly lowered (Pfeifer and Galambos, 1965). These apparent discrepancies observed with $\alpha$-mDopa may be related to the presence of false transmitters (e.g., $\alpha$-methyl-dopamine and $\alpha$ methyl-norepinephrine) which are resistant to reserpine action (Carlsson and Lindqvist, 1962; Carlsson et al., 1965). That such false transmitters can influence the sensitivity of animals to seizures is demonstrated by the ability of metaraminol (a metabolite of $\alpha-\mathrm{mmT}$ ) to potentiate acetazolamide action if the catecholamine is administered 30 min. before the test. However, if metaraminol is given $4 \mathrm{hr}$. prior to testing, it antagonizes acetazolamide action. These effects could be due to release of endogenous catecholamines by metaraminol, resulting in an initial elevation of norepinephrine and subsequent prolonged depletion of endogenous catecholamines (Carlsson, 1964). Release of catecholamines by metaraminol would elevate the amount of these amines at effector sites and in accordance with the hypothesis, should lower susceptibility to seizures. On the other hand, depletion of these 
amines by metaraminol would reduce the catecholamine activity at receptor sites and cause an increase in seizure responsiveness. Such mechanisms would explain the biphasic effect seen with metaraminol on the anticonvulsant action of acetazolamide.

Alpha-methy1-p-tyrosine $(\alpha-m T)$ is a potent in vitro inhibitor of tyrosine hydroxylase (Nagatsu et al., 1964). Evidence accumulated by Spector et al. (1965) further shows that $\alpha-m T$ lowers brain catecholamine levels and probably does so by in vivo inhibition of tyrosine hydroxylation - the rate limiting step in the biosynthesis of catecholamines from tyrosine (Hess et al., 1961). Weissman and Koe (1965) reported that $\alpha-\mathrm{mT}$ depleted dopamine and norepinephrine in rat brain with peak action between 5 and $7 \mathrm{hr}$. Additional histochemical and biochemical studies show that the compound lowered norepinephrine and dopamine in various forebrain regions, $4 \mathrm{hr}$. after injection (Anden et al., 1966a; Anden, 1967). In support of the hypothes is that low norepinephrine level increases seizure susceptibility, $\alpha$-mT treatment increases the sensitivity of animals to maximal seizures induced by electroshock (Rudzik and Mennear, 1966a; 1966b). Thresholds to electrically-induced extensor seizures are also depressed (Chen et al., 1968a). This drug also reduces thresholds in mice pretreated with diphenylhydantoin or metazolamide. In this respect, $\alpha-m T$ is different from the decarboxylase inhibitors, $\alpha$-mmT and $\alpha$-mDopa. However, the effect of $\alpha-m$ T on seizure threshold could also be antagonized by iproniazid (Chen et al., 1968a). In the same study, monoamine oxidase per se had no effect. 
Dopamine- $\beta$-hydroxylase, the enzyme which converts dopamine to norepinephrine can be inhibited by disulfiram, in vivo (Goldstein, 1966). The effect of this compound on seizures induced by $m$-fluorotyrosine was evaluated by Weissman and Koe (1967), who reported no significant change in seizure susceptibility after disulfiram.

Endogenous catecholamine levels may also be elevated by treatment with the amino acid precursor, 1-dopa, which readily penetrates the blood-brain-barrier (Carlsson, 1964). The compound is decarboxylated to dopamine which in turn is $\beta$-hydroxylated to norepinephrine (Carlsson, 1964; Euler, 1968). By itself, dopa generally produces no alteration in seizure susceptibility. However, in the presence of monoamine oxidase inhibition, dopa raises thresholds to electricallyinduced extensor seizures (Chen et al., 1968a). Lessin and Parkes (1959) failed to see a significant action of dopa on survival time of mice on exposure to metrazol, even in the presence of iproniazid treatment. However, DeSchaepdryver et al., 1962, reported an increase in threshold to electroshock seizures following 1-dopa injection in animals pretreated with reserpine and iproniazid. Antagonism of hydrazide-induced seizures after dopa and iproniazid treatment was also observed by Balzer et al., 1960. Whether such pharmacological manipulations result in a selective increase in dopamine or norepinephrine or both has been debated. However, more direct evidence demonstrating norepinephrine involvement in seizure expression was presented by Jones and Roberts (1968). By intraventricular injection, these workers found that low doses of dopamine depressed 
seizure threshold to metrazol convulsions. The threshold was returned to normal with high doses. It was suggested by Jones and Roberts that with the low dose, the action seen with dopamine was a direct one, whereas at the higher doses conversion to norepinephrine returned seizure threshold to normal. This explanation is consistent with the hypothesis that presence of norepinephrine reduces susceptibility to seizures.

The level of endogenous catecholamines can also be elevated by interference with the in vivo catabolism of such compounds. Since these amines are metabolized by monoamine oxidase and catechol-omethyl transferase, inhibition of their activities would increase catecholamine levels in the intact animals (Pletscher, 1967b; Kopin, 1964; Koel1e, 1965). However, the effect of monoamine oxidase inhibitors is complex. Some workers reported an anticonvulsant action (Chow and Hend7ey, 1959; Prockop et al., 1959a; 1959b; Yen et al., 1962). Other investigators observed either no action or a facilitation of seizures (Kobinger, 1958; Spoerlein and Ellman, 1961; DeSchaepdryver et a1., 1962; Samsome and Dell Omodarme, 1963). More recently, Turner and Spencer (1968) who studied the response of metrazol seizures in mice and rats to several monoamine oxidase inhibitors reported a difference in effect between individual drugs. They saw a transient proconvulsant effect with iproniazid, phenelzine and tranycypromine, but no action with nialamid and pargyline. The doses employed were sufficient to inhibit monoamine oxidase. However, they noted that those agents with proconvulsant action also had sympathomimetic effects. 
Lehman (1967) who worked with the monoamine oxidase inhibitors, isocarboxazid, trancypromine and nialamid on audiogenic seizure pattern saw protection against all phases (tonic, clonic and "wild running") of such seizures. A11 3 compounds provided 90 to $95 \%$ protection against the tonic-clonic seizures. In combination with catecholamine depleting agents, monoamine oxidase inhibitors generally antagonized the seizure facilitation of such compounds (e.g., tetrabenazine, reserpine, $\alpha-m m T$ and $\alpha-m T)$. With precursors of norepinephrine, these agents generally elevated seizure threshold, as shown above.

Pyrogal101 inhibits the activity of catechol-0-methyltransferase (COMT) which is responsible for the breakdown of circulating catecholamines (Axelrod et al., 1958; Carlsson, 1964; Izquierdo et al., 1964). Treatment with this agent would produce an elevation of endogenous catecholamines (Crout et al., 1961; Matsuka et al., 1962; Maitre, 1966). Employing this compound, Lehman (1967) saw a $69 \%$ decrease in tonic seizure incidence, $22 \%$ protection against the clonic phase of the seizure and $9 \%$ total protection against audiogenic seizures. This action was enhanced in animals treated also with a monoamine oxidase inhibitor (e.g., nialamid, trancypromine or isocarboxazid). These results support the hypothesis that elevation of norepinephrine concentration reduces susceptibility to seizures. However, the interpretation of data with pyrogallol must be cautious since it has other actions besides COMT inhibition (Carlsson, 1964). 
Endogenous catecholamine levels may also be elevated by inhibition of "reuptake" of these amines into axonal terminals. This is one of the mechanisms postulated to end the action of catecholamines in vivo (Carlsson and Waldeck, 1965; Glowinski and Axelrod, 1965; 1966). Investigators, who studied the effects of drugs which inhibit "reuptake" of catecholamines on seizure susceptibility also presented data in support of the above hypothesis. For example, imipramine was reported by Glowinski et al. (1966) to increase the free physiological active norepinephrine through blockade of "reuptake". Using this substance, Lehman (1967), observed $97 \%$ protection of the tonic phase of seizures in audiogenic seizure susceptible mice. Chen et al. (1968a) confirmed these findings with electroshock extensor seizures. They further showed that reserpine, tetrabenazine and dopa potentiated imipramine action. Examination of other imipramine-like drugs, e.g., desipramine, amitriptyline, deoxyephedrine and cocaine, essentially produced the same results (Lehman, 1967; Chen et al., 1968a). These agents also block amine "reuptake" (Carlsson and Waldeck, 1965; Chen et al., 1965; Glowinski and Axelrod, 1966).

Seizure susceptibility is also altered following treatment with sympathomimetic agents. For example, oral administration of amphetamine elevated thresholds to electroshock (Tainter et al., 1943; Frey, 1964), while sma11 doses of amphetamine increased convulsive thresholds of mice to hexafluorodiethyl ether seizures (Wolf and Stock, 1966). Moreover, amphetamine suppressed tonic seizures and provided $50 \%$ total protection against audiogenic seizures (Lehman, 
1967). Brain norepinephrine was decreased by this substance, but normetanephrine formation was elevated, demonstrating a greater release of endogenous catecholamines (Glowinski et al., 1966). In addition, amphetamine inhibits uptake of norepinephrine in vivo (Axelrod and Tomchick, 1960). Both mechanisms tend to raise catecholamine concentrations at effector sites and could probably account for the anticonvulsant effect of amphetamine. Other workers (Frey, 1964; Wolf and Stock, 1966) attributed amphetamine-induced alleviation of seizures to a desynchronizing action in the frontal cortex (Longo and Silvestrini, 1957) and to its inhibitory action at the level of the reticular formation (Xavier and Timo-Iaria, 1964).

However, amphetamine also exhibits a proconvulsant action. Moore and Lariviere, 1964, observed enhanced lethality in grid shocked animals following amphetamine injection. Other workers (Wolf and Stock, 1966) reported an increase in susceptibility to metrazol seizures. A more recent study (Turner and Spencer, 1968) demonstrated marked potentiation of metrazol convulsant and lethal effects by amphetamine (30 to $90 \mathrm{~min}$. after injection) in mice. However, $6 \mathrm{hr}$. after its administration, a definite anticonvulsant effect was observed. According to Turner and co-workers such findings could be related to a true postictal effect related to earlier intense central adrenergic stimulation, produced by amphetamine. A possible explanation for the proconvulsant action of amphetamine could be its ability to lower norepinephrine level in the brain (Moore and Lariviere, 1964), resulting in less activation at effector sites. Such a mechanism is 
consistent with the hypothesis that norepinephrine depresses seizure responsiveness.

An involvement of norepinephrine in seizure expression is also demonstrated from metabolic studies. Kety et al. (1967) measured the "turnover" (defined as the synthesis and utilization) of tritiated norepinephrine in rats subjected to chronic maximal electroshock seizures. The radioactive compound was injected intracisternally. These workers reported that tritiated norepinephrine was similar in concentration in the electroshocked and untreated animals, 30 min. after incubation with the radioactive compound. However, after $5 \mathrm{hr}$. incubation, the tritiated norepinephrine level was less in the electroshocked group, indicating that the rate of disappearance was enhanced in this group. From these data, Kety et al. (1967) inferred that chronic maximal electroshock treatment increased the "turnover" of norepinephrine in vivo. They based their explanation on the evidence that intracisternal injection of tritiated norepinephrine rapidly labels endogenous stores in the brain quite specifically. The resulting radioactive norepinephrine declines at a rate indicative of the endogenous norepinephrine "turnover" (Glowinski and Axelrod, 1966; Iversen and Glowinski, 1966).

A similar study by Landisch et al. (1969) confirmed the above observations. These workers demonstrated no difference in monoamine oxidase activity (measured with serotonin as the substrate) in the electroshocked rats compared to the untreated animals. A1so, electroshock produces a slower increase in norepinephrine in relation to a 
fall of radioactive metabolites with time. From these observations, they suggested that the increase in "turnover" of norepinephrine following chronic electroshock treatment was related to a disturbance in the storage mechanism. Further support for an increased "turnover" of norepinephrine is demonstrated by an elevation in rat brain tyrosine hydroxylase activity after electroconvulsive shock (Musacchio et al., 1969).

Changes in catecholamine levels subsequent to electroshock have also been observed by other investigators. Some reported a release in brain and adrenal catecholamines (Maynert and Levi, 1964). Others saw an elevation of brain norepinephrine and dopamine (Satoh et al., 1964). A change in cerebral dopamine metabolism was reported by Cooper et al. (1968), while elevation in dopamine synthesis was seen by Engel et al. (1968).

Over the last few years reports linking the anticonvulsant action of carbonic anhydrase inhibitors (acetazolamide, metazolamide) to catecholamines have been presented. Gray et al. (1963) saw an antagonism of acetazolamide and metazolamide action by reserpine. Administration of dopa or monoamine oxidase inhibitors reversed this action. Rudzik and Mennear (1966a; 1966b) reported antagonism of acetazolamide and metazolamide action by other catecholamine depleting agents, $\alpha-m T$ and R04-1284. The antagonism by reserpine is prevented by $\alpha$-mDopa, which blocks the depletion of brain norepinephrine by reserpine (Pfeifer and Galambos, 1965). 
The anticonvulsant action of other agent, e.g., carbon dioxide, has also been altered by pharmacological manipulations of catecholamine concentrations. Faulkner and Mennear (1969) reported that reserpine, $\alpha-m T$ and $\alpha-m m T$ antagonized the anticonvulsant action of carbon. dioxide in maximal electroshock seizures.

Any change in endogenous catecholamine levels results in a corresponding alteration of adrenergic stimulation at effector sites. This could also be achieved by interfering with the actions of catecholamines at their receptor sites. According to the traditional classification of Ahlquist (1948) catecholamine actions in cardiac and smooth muscles may be differentiated into alpha and beta actions. Such a characterization is obtained by a study of tissue responses to various sympathomimetic amines like epinephrine, norepinephrine, phenylephrine and isoproterenol. The alpha receptors mediate responses where the order of potency is epinephrine $>$ norepinephrine $>$ phenylephrine > isoproterenol. Beta receptors, on the contrary, mediate responses for which the order of potency is isoproterenol > epinephrine> norepinephrine > phenylephrine (Ahlquist, 1948; 1958; Furchgott, 1967). In addition, the responses mediated through alpha receptors can be selectively antagonized by a variety of natural and synthetic agents (e.g., ergot alkaloids, phentolamine and phenoxybenzamine) while responses mediated through beta receptors can be selectively antagonized by a number of synthetic agents (e.g., dichloroisoproterenol (DCI), pronetha101, propranolol, sotalol (MJ1999) and INPEA). These compounds are referred to as alpha or beta adrenergic blocking agents (Nickerson 
and Goodman, 1947; Harvey and Nickerson, 1954; Nickerson, 1965; Moran, 1967; Nickerson, 1967).

The effects of adrenergic blocking compounds on seizure susceptibility have been investigated by a few workers. Phenoxybenzamine and phentolamine antagonize the anticonvulsant effect of acetazolamide and lower the thresholds to electrically-induced extensor seizures (Rudzik and Mennear, 1966a; Chen et al., 1968a). Mice are also made more susceptible to low frequency electroshock seizures following phenoxybenzamine treatment (Yeoh and Wolf, 1968). Hence the alpha adrenergic blocking agents seem to increase the susceptibility of animals to seizures.

The data obtained with the beta adrenergic blocking agents are not so clear-cut. This is probably due to the fact that such compounds have other actions besides beta receptor blockade (Raper and Wale, 1968). Propranolo1, pronetha101, dichloroisoproterenol and $D(-)$-INPEA possess blocking actions on alpha receptors as we11 (Ariens, 1967; Kohli and Ling, 1967; Gulati et al., 1969). Further, both propranolo1 and pronethalol have local anesthetic action (Morales-Aguiers and Vaughan-Williams, 1965). Pronethatol and DCI have sympathomimetic actions (B1ack and Stephenson, 1962) and propranolol directly depresses the central nervous system at high doses (Black et al., 1964). Moreover, beta blocking agents like their alpha counterparts also inhibit the "reuptake" of catecholamines into axonal terminals, a reaction which is important in terminating catecholamine action (Moreira and Osswald, 1965). Rudzik and Mennear (1966a; 1966b) reported that DCI 
increased the anticonvulsant action of acetazolamide on maximal electroshock seizures while sotalol antagonized its action. Sotalol also opposes the anticonvulsant action of metazolamide and reduces seizure thresholds of mice to electrically-induced extensor seizures of the hind 1 imbs. In contrast, pronethalol reduces the susceptibility of animals to maximal electroshock (Murmann et al., 1966; Chen et a1., 1968a), low frequency electroshock (Yeoh and Wolf, 1968) and metrazolinduced seizures (Murmann et al., 1966). This substance also potentiates the anticonvulsant action of metazolamide and acetazolamide (Mennear and Rudzik, 1968; Chen et al., 1968a). Seizure susceptibility is also reduced by propranolol (Murmann et al., 1966; Yeoh and Wolf, 1968). However, both $D(-)$ and $L(+)$ INPEA fail to alter susceptibility to metrazol, nicotine, strychnine and tonic electroshock seizures (Murmann et al. , 1966). D(-) INPEA possesses significantly greater ability than its $L(+)$ isomer to block beta adrenergic actions (Almirante and Murmann, 1966; Pati1, 1968). These studies show that the effects of beta adrenergic agents on the susceptibility of animals to seizures range from a proconvulsant effect, no action to protection from seizures.

In summary, this survey reveals an involvement of endogenous catecholamines in seizure expression. A reduction of these neurotransmitters by interference with either their synthesis or their storage produces animals which are more responsive to seizures. In such subjects, susceptibility to seizures is decreased when intraneuronal metabolism of these amines is prevented. Inhibition of extraneuronal inactivation of catecholamines or blockade of "reuptake" of these sub- 
stances into axonal terminals also protects animals from seizures. Moveover, elevation of endogenous catecholamines by direct administration of these compounds or their precursor, dopa, reduces seizure susceptibility of animals.

However, not all the available data support the hypothesis that the presence of norepinephrine reduces the responsiveness of animals to exhibit seizures. Thus, as shown above, contradictory reports were presented by workers who examined seizure susceptibility after different pharmacological manipulations of endogenous catecholamine levels. These inconsistencies may have been introduced by the wide variety of seizures investigated; differences between animals used (species and strain variations) and complications arising from drug-drug interactions where chemoshock seizures are used. Moreover, in many procedures stress of the experimental animal due to brief restraint may have confounded the data (Swinyard et al., 1962). Perhaps, the most probable source of disagreement between experimental findings could be traced to the fact that many investigators only studied one dose of the test compound. Since drug actions are generally dose dependent, a more lucid picture of these actions would be obtained by studying multiple, non-toxic doses. The time of test subsequent to drug injection could be critical and may contribute to differences in experimental observations. For example, Mennear and Rudzik (1968) found that pronethalol potentiated the anticonvulsant action of acetazolamide at 15 min., but antagonized its anticonvulsant effect when administered 60 min. prior to the test. 
The present studies were undertaken in an attempt to clarify these discrepancies and to reach a better understanding of the relationship of endogenous catecholamines to seizure susceptibitity.

Seizures were induced in freely moving animals by electrical stimulation of the hippocampus. As demonstrated in the previous section, such a procedure avoided complications that could arise from druginduced seizures and stress introduced by briefly restraining animals for the application of electroshock. These sefzures were shown to respond to diphenylhydantoin and trimethadione, two prototype anticonvulsants, in the same way as other experimentally produced minimal seizures.

Examination of the hypothesis that increased cerebral concentrations of norepinephrine reduces the responsiveness of animals to seizures was approached in three ways. First, the effect of catecholamine depletion on seizure susceptibility of rats was studied employing reserpine and $\alpha-m T$. These agents were examined for their ability to alter seizure threshold when administered alone and in combination. If the stated hypothesis is valid, reserpine, $\alpha-m$ T or combination of these two drugs should depress seizure threshold, since they both produce depletion of brain catecholamines (Nagatsu et al., 1964; Glowinski et al., 1966; Rech et al., 1966a, 1966b; 1967).

Second, the effect of a selective increase in central norepinephrine on seizure threshold in rats was examined. Such studies were conducted in normal rats as well as in animals where endogenous catecholamines were markedly lowered with reserpine plus $\alpha-m$ T. For 
this purpose the amino acid precursor of norepinephrine, DL-threo-3,4dihydroxyphenylserine (DL-threo-DOPS) was used because it readily penetrates the blood-brain-barrier (Carlsson, 1964). Although this compound is probably not a physiological precursor of norepinephrine (Holtz, 1959; Carlsson, 1964), it is decarboxylated in vivo by dopa decarboxylase to 1-norepinephrine (Carlsson, 1964; Torchiana et al., 1966; Creveling et al., 1968). DL-threo-DOPS restores norepinephrine but not dopamine levels in animals treated with $\alpha-m T$ (Corrodi and Fuxe, 1967; Creveling et al., 1968) or reserpine (Carlsson, 1964; Torchiana et al., 1966; Creveling et al., 1968). In this respect, DL-threo-DOPS is a more selective precursor of norepinephrine than is dopa. This latter compound forms dopamine on decarboxylation. Dopamine is in turn converted to norepinephrine by dopamine- $\beta$-oxidase in vivo (Pletscher, 1957b; Carlsson, 1964; Goldstein, 1966). By using DL-threo-DOPS in animals depleted of both norepinephrine and dopamine, selective restoration of 1-norepinephrine can be achieved. This principle was employed in the present study. If norepinephrine indeed suppresses the expression of seizures, DL-threo-DOPS per se should raise seizure threshold. This compound should also be capable of antagonizing the seizure facilitation caused by reserpine and $\alpha-\mathrm{mT}$, administered in combination.

The third approach to the hypothesis that cerebral norepinephrine attenuates the precipitation of seizures was to examine the effect of several alpha and beta adrenolytic agents on seizure threshold. These compounds alter in vivo adrenergic tone by producing blockade at 
adrenergic effector sites (Nickerson and Goodman, 1947; Nickerson, 1967). In the periphery, norepinephrine acts predominentiy on alpha adrenergic receptors (Ahlquist, 1948; 1958; Furchgott; 1967). Its action is readily antagonized by alpha adrenergic blocking agents like phenoxybenzamine and phentolamine. If norepinephrine indeed suppresses seizure susceptibility, then alpha adrenergic receptor blockade should lower seizure threshold. In order to examine this aspect of the problem, the effects of phenoxybenzamine and phentolamine at several non-toxic doses were tested.

Although norepinephrine acts mainly on alpha adrenergic receptors, it also has slight action on beta adrenergic sites (Furchgott, 1967). Furthermore, as shown earlier, beta adrenergic blocking compounds can alter the sensitivity of animals to seizures. Most of the studies show that such compounds protect animals against convulsions, but data indicating the reverse effect (a proconvulsant action) have also been obtained. A clarification of this discrepancy was attempted in this study through an evaluation of several non-toxic doses of four established beta adrenergic blocking agents on seizure threshold. The drugs chosen were propranolo1, pronethalo1, sotalol and $D(-)$ INPEA. The $L(+)$ isomer of INPEA was examined for its effect on seizure susceptibility, in order to elucidate further the involvement of beta adrenergic receptors in seizure expression. As indicated earlier, the $L(+)$ isomer has relatively weak adrenergic blocking action on beta receptors unl ike $D(-)$ INPEA. 
From these studies, the relationship of norepinephrine to the responsiveness of rats to seizures induced by electrical stimulation of the hippocampus should be demonstrated. Furthermore, the data obtained from tests utilizing adrenergic blocking agents should reveal any involvement of central alpha or beta receptors in the precipitation of such seizures.

\section{B. Methods}

The animals used and the procedure for evaluating seizure threshold were the same as previously described (see GENERAL PROCEDURE). A11 drugs were administered by intraperitoneal injection. Propranolo1, pronethalol, sotalol, $D(-)$ and $L(+)$ INPEA were dissolved in saline while phentolamine was dissolved in distilled water. These drugs were injected at a volume of $1 \mathrm{ml} . / \mathrm{kg}$. Phenoxybenzamine, $\alpha-\mathrm{mT}$ and DL-threo-DOPS were suspended in 10\% propylene glycol in saline with the aid of a high frequency generator (Fisher). The volume injected was $3 \mathrm{ml} . / \mathrm{kg}$. Reserpine (Serpasil, Ciba) was given in a volume of $0.8 \mathrm{ml} . / \mathrm{kg}$.

Since animals were used repeatedly for drug studies in this procedure, care was taken to employ only doses of drugs which were non-toxic to the animals. At least two weeks elapsed between drug injections. Reserpine was studied at a dose of $2 \mathrm{mg} . / \mathrm{kg}$., given $24 \mathrm{hr}$., prior to test. Alpha-methyl-p-tyrosine was injected in 3 doses of $50 \mathrm{mg} . / \mathrm{kg}$. at $4 \mathrm{hr}$. intervals and seizure threshold was evaluated $12 \mathrm{hr}$. after the first injection. These schedules were selected based on the findings of previous investigations (Moore et al., 1967; Rech et al., 
1967; Carr and Moore, 1968), who showed that such multiple injections of $\alpha-m T$ produced significant depletion of brain catecholamines while overcoming the renal toxicity due to high doses of the drug. These authors also used combination treatment with reserpine and $\alpha-m T$ at the stated doses and the time of treatment used in the current study. DL-threo-DOPS was used in a dose of $200 \mathrm{mg} . / \mathrm{kg}$. , injected $1 \mathrm{hr}$. before evaluation of seizure threshold. This was based on the study by Corrodi and Fuxe (1967), who found that this dose returned brain norepinephrine to $50 \%$ of the normal level in $\alpha-m T$ treated animals where its concentration was initially reduced to about $20 \%$ of normat.

Such a protocol was only selected in this study after initial histochemical evaluations had confirmed that norepinephrine content in the brain was indeed altered under these experimental conditions. Since the concentration of brain norepinephrine after $2 \mathrm{mg} . / \mathrm{kg}$. in the rat is still significantly lowered 6 days after injection (Carr and Moore, 1968), the cross-over design discussed under Methods in Chapter III, was not employed in studies where reserpine was used. When adrenergic blocking agents were employed, the same procedures as previously described for diphenylhydantoin and trimethadione were used. Selection of the doses and the treatment time were based on neurotoxicity studies of these agents as described in GENERAL PROCEDURES. A11 doses were less than the toxic dose 50 of the drug.

The number of animals employed in the seizure threshold studies varied from 6 to 10 . In histochemical studies, 2 rats per group were 
examined, while 4 animals per group were employed in the biochemical assay for norepinephrine.

Histochemical evaluations of norepinephrine in specific brain areas were conducted using the modified procedure of Falck and Owman (1965). This is based on a ring closure due to condensation of biogenic amines with formaldehyde gas to form green (catecholamines) or yellow (serotonin) fluorescent products. Dopamine and norepinephrine form 6,7-dihydroxy-3,4-dihydro-isoquinolines which have main peaks of activation and emission at 410 and $480 \mathrm{mu}$, respectively. Examination of brain sections were conducted with the aid of the rat atlas of Konig and Klippel (1963) and the classification of brain aminergic neurons described by Dahlstrom and Fuxe (1964). A detail of the actual procedure used is presented in the APPENDIX. In this method, norepinephrine contents in the brains of rats were examined after treatment with reserpine per se, $\alpha-m$ Ter se, reserpine plus $\alpha-m T$ and reserpine plus $\alpha-m$ T plus DL-threo-DOPS. The results were recorded on photomicrographs.

Norepinephrine in specific areas of the brain was also assayed by means of a modified method of Saelens et al. (1967). Dissection of the brain regions followed the procedure of Glowinski and Iversen (1966). Norepinephrine was analyzed in the brain stem (pons-medulla oblongatal, hypothalamus, midbrain, hippocampus, corpus striatum and the neocortex. Rats treated with reserpine plus $\alpha-m T$, reserpine 
plus $\alpha-m T$ plus DL-threo-DOPS, DL-threo-DOPS per se and untreated änimals were evaluated for brain norepinephrine contents. This assay was based on the principle that norepinephrine in brain homogenate is converted to epinephrine- $\mathrm{N}-$ methy $1-14 \mathrm{C}$ in the presence of S-adenosylmethionine-methy $1-14 \mathrm{C}$ and phenylethanolamine-N-methyltransferase. Separation of radioactive epinephrine from the S-adenosylmethioninemethy $1-14 C$ was achieved by paper chromatography. The radioactive epinephrine was developed by alternate exposure of the dried chromatogram to ammonia and jodine vapors. The stained areas were cut out, extracted with $100 \%$ ethanol and counted in Packard Tri-Carb 1 iquid scintillation spectrometer using a toluene based phosphor. The detailed procedure employed in the analysis is described in the APPENDIX.

The results were expressed as $\mathrm{ng} \cdot / \mathrm{gm}$. brain tissue. These data were then subjected to analyses of variance and to the student's " $t$ " test (Goldstein, 1964b). The mean and the 95\% confidence 1imits were determined. Comparisons of norepinephrine concentrations between different treatment groups in each brain region were conducted. The difference between any two groups was considered significant at $P<0.05$. 


\section{Results}

The involyement of catecholamines in seizures induced by electrical stimulation of the hippocampus was demonstrated by changes in seizure thresholds in rats which had been treated with reserpine, $\alpha-m T$ and DL-threo-DOPS. Depletion of endogenous catecholamines is produced by reserpine and $\alpha-m T$, while DL-threo-DOPS is an immediate precursor of 1-norepinephrine and is therefore capable of restoring this neurotransmitter in rats depleted of catecholamines. The results obtained with these drugs are presented in Figure 5. As indicated previously, threshold ratio was calculated by dividing the seizure threshold after drug or vehicle treatment by the the seizure threshold before drug treatment. Any change in seizure susceptibility after drug administration in rats would be represented by a significant alteration of threshold ratio from $1.0(P<0.05)$.

Reserpine (2 mg./kg., given $24 \mathrm{hr}$. prior to test) reduced seizure threshold by about $50 \%$. The control studies with this group of rats showed no significant change in seizure threshold. All the drug treated animals appeared sedated and lost weight. Five out of six test rats had ptosis and diarrhea. When placed in the test chamber, little exploratory behavior was exhibited by reserpinized rats. They sat in a "hunch" position with eyes closed and showed occasional fore 1 imb movements resembling facial grooming. These animals could be aroused with difficulty by repeated tapping on the side of the test chamber. At threshold current, 2 out of 6 animals exhibited maximal seizures (tonic hind limb extension! with immediate unconsciousness and death in spite of 
Figure 5. The importance of adrenergic tone to seizure threshold. Threshold Ratio was determined by dividing the drug seizure threshold or the control seizure threshold by the stable seizure threshold. The vertical bars denote $95 \%$ confidence limits. $\Delta$, reserpine; $\Delta, \alpha-m T ; \Delta$, reserpine and $\alpha-m T ; 0$, reserpine $+\alpha-m T+D L-$ threoDOPS; $\square$, DL-threo-DOPS; 0 , control. 


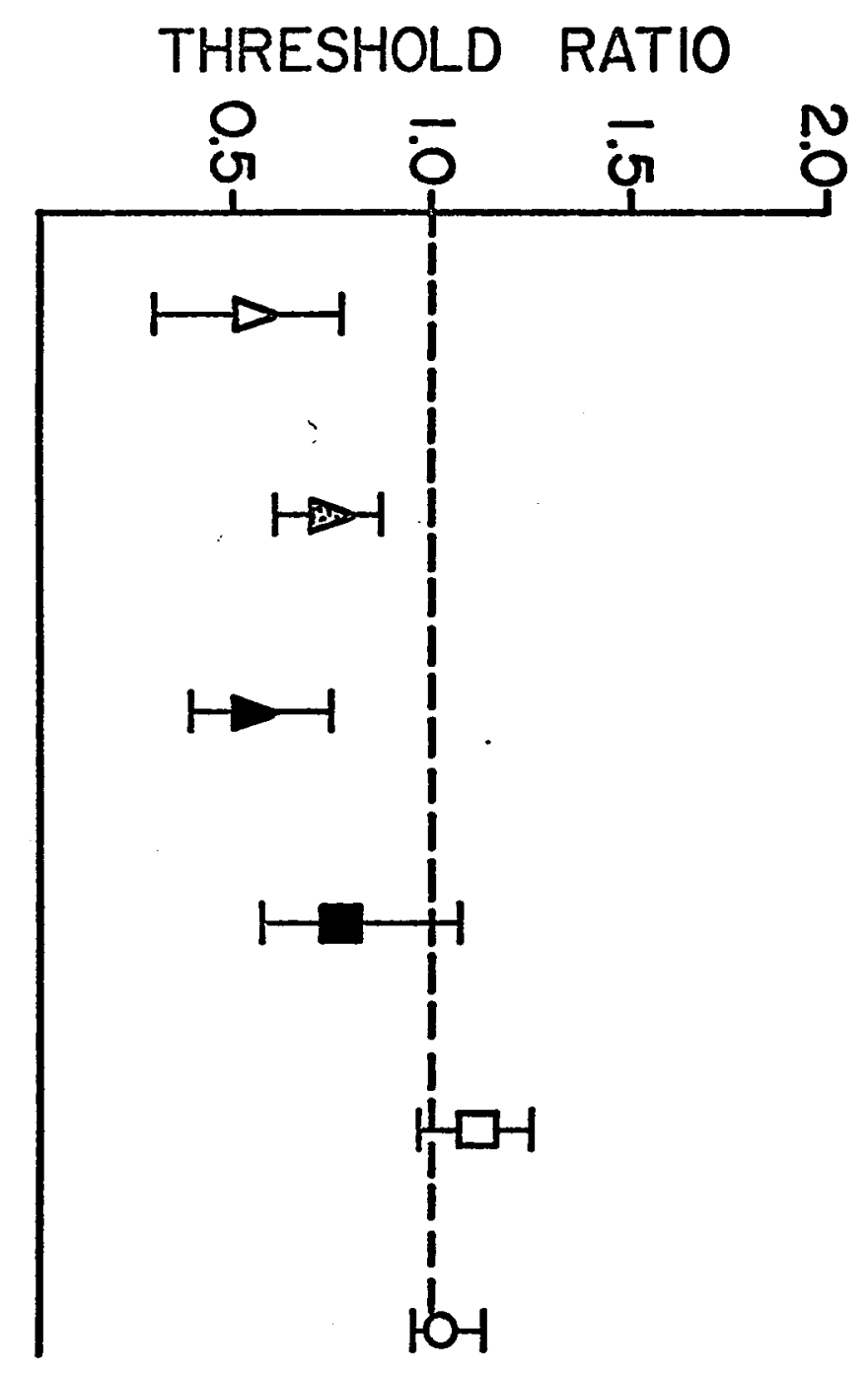


heart massage and artificial respiration. The other rats showed slight rhythmic movements of the jaws with administration of current very much lower than the predetermined stable seizure threshold. The recovery from seizures in reserpinized rats was difficult to assess since these animals were sedated and did not resume any exploratory behavior after the seizures. When maximal seizures were exhibited in reserpine treated animals they were very sudden and lacked the clonic component.

The mean seizure threshold in rats treated with 3 doses of $50 \mathrm{mg} . / \mathrm{kg} . \quad \alpha-\mathrm{mT}$ injected at intervals of $4 \mathrm{hr}$. was reduced by $30 \%$. Saline injections in these animals failed to induce any change in seizure susceptibility. Of the 7 animals tested, only 1 had diarrhea. Sedation occurred in 3 of the rats, while the remaining animals showed normal exploratory behavior and grooming when placed in the test chamber. One of the centrally depressed animals was hypersensitive to handling after the test. In all these animals the seizures exhibited at threshold current were minimal seizures.

An additional study conducted with injections of 3 doses of $80 \mathrm{mg} . / \mathrm{kg} . \quad \alpha-\mathrm{mT}$, did not produce any further lowering of seizure threshold. In fact, a depression of onty $20 \%$ was observed in such rats. However, gross observation of these test animals indicated that they were all sedated and lacked exploratory behavior when placed in the test environment. Piloerection was present in 6 out of 7 rats, demonstrating possible hypothermia. Moreover, 2 of the test animals "indiscriminately"ate their own feces. 
Unlike reserpine, the lower dose of $\alpha-m T$ did not cause animals to lose weight. Some weight loss was, however, seen in rats which received the larger dose of $\alpha-m T$. Since no further reduction in seizure threshold was obtained with the higher dose of the tyrosine hydroxylase inhibitor, and since a greater degree of behavioral disturbance occurred with this dose, $\alpha-m T$ was given in 3 doses of 50 $\mathrm{mg} . / \mathrm{kg}$. each in all subsequent studies.

When both reserpine and $\alpha-m$ T were administered to rats, a $50 \%$ reduction in seizure threshold was produced. Compared to the effect of reserpine per se, there was no quantitative difference between the degree of seizure threshold depression. The rats which were treated with this combined schedule were sedated, exhibited ptosis, diarrhea and were deficient in exploratory behavior. Considerable loss of weight also occurred. Maximal seizures, beginning with a very sudden convulsion, followed by tonic hind limb extension, unconsciousness and death were induced in all animals at threshold current. Death occurred despite heart massage and artificial respiration. Control studies conducted a week prior to the drug experiments confirmed that stable seizure thresholds could be reliably reproduced in animals after multiple injections of the drug vehicles.

DL-threo-DOPS increased the mean seizure threshold of reserpine and $\alpha-m T$ treated animals by $25 \%$ (Figure 5 ). The upper $95 \%$ confidence limit of the threshold ratio was greater than 1.0, indicating a tendency of the amino acid to restore seizure threshold to normal. Five of the 6 animals tested, exhibited maximal seizures. The gross behavior of 
catecholamine depleted rats was not altered by DL-threo-DOPS. They were sedated, had little exploratory behavior, exhibited ptosis and had faster respiratory rates when compared to the depleted animals. Three out of 6 rats tested died following seizures, in spite of heart massage and artificial respiration. This indicates some antagonism of seizure severity since $100 \%$ lethality was observed in catecholamine depleted rats under similar experimental conditions.

When administered to naive rats, DL-threo-DOPS elevated seizure threshold in 3 out of 7 animals, but this effect was not significant as shown in Figure $5(P<0.05)$. These animals exhibited little exploratory activity when they were in the test chamber. Instead, they were inactive and were often seen sleeping in an unusual posture, characterized by lying on their sides with backs and legs extended. Havlicek (1967), who also administered DL-threo-DOPS to rats made a similar observation. In comparison to reserpine treated animals, these rats were easier to arouse by repeated tapping on the test chamber.

The extent of norepinephrine alteration subsequent to various drug treatments was determined by histochemical and biochemical methods. Histochemical observations were recorded on photomicrographs at a magnification of 160. Representative samples of these photomicrographs ares presented in Plates II to VI. The effects of pharmacological manipulations on the norepinephrine neurons in the tunica adventitia of the basilar artery are seen in Plates II, III and IV. The untreated specimen demonstrated the presence of brilliant green fluorescence 
Plate II. Histochemical studies of drug effects on central norepinephrine neurons.

Top. Normal rat. The basilar artery in the region of the mid-brain. Presence of norepinephrine neurons is demonstrated by marked fluorescence (green) in the tunica adventitia of the artery. Magnification X 160 .

Bottom. Reserpine treated rat. Absence of fluorescence in the tunical adventitia of the basilar artery indicates depletion of catecholamines by reserpine. Magnification $\times 160$. 


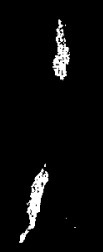

s* $\infty \quad \therefore$ 
Plate III. Histochemical studies of drug effects on central norepinephrine neurons.

Top. $\quad \alpha-m T$ treated rat. Presence of intense green fluorescence in the tunica adventitia of the basilar artery indicates no depletion of catecholamines in the neurons. Magnification $\times 160$.

Bottom. Reserpine $+\alpha-m T$ treated rat. Absence of fluorescence in the tunica adventitia of the basilar artery indicates marked depletion of catecholamines produced by administration of these two drugs. Magnification $\times 160$. 


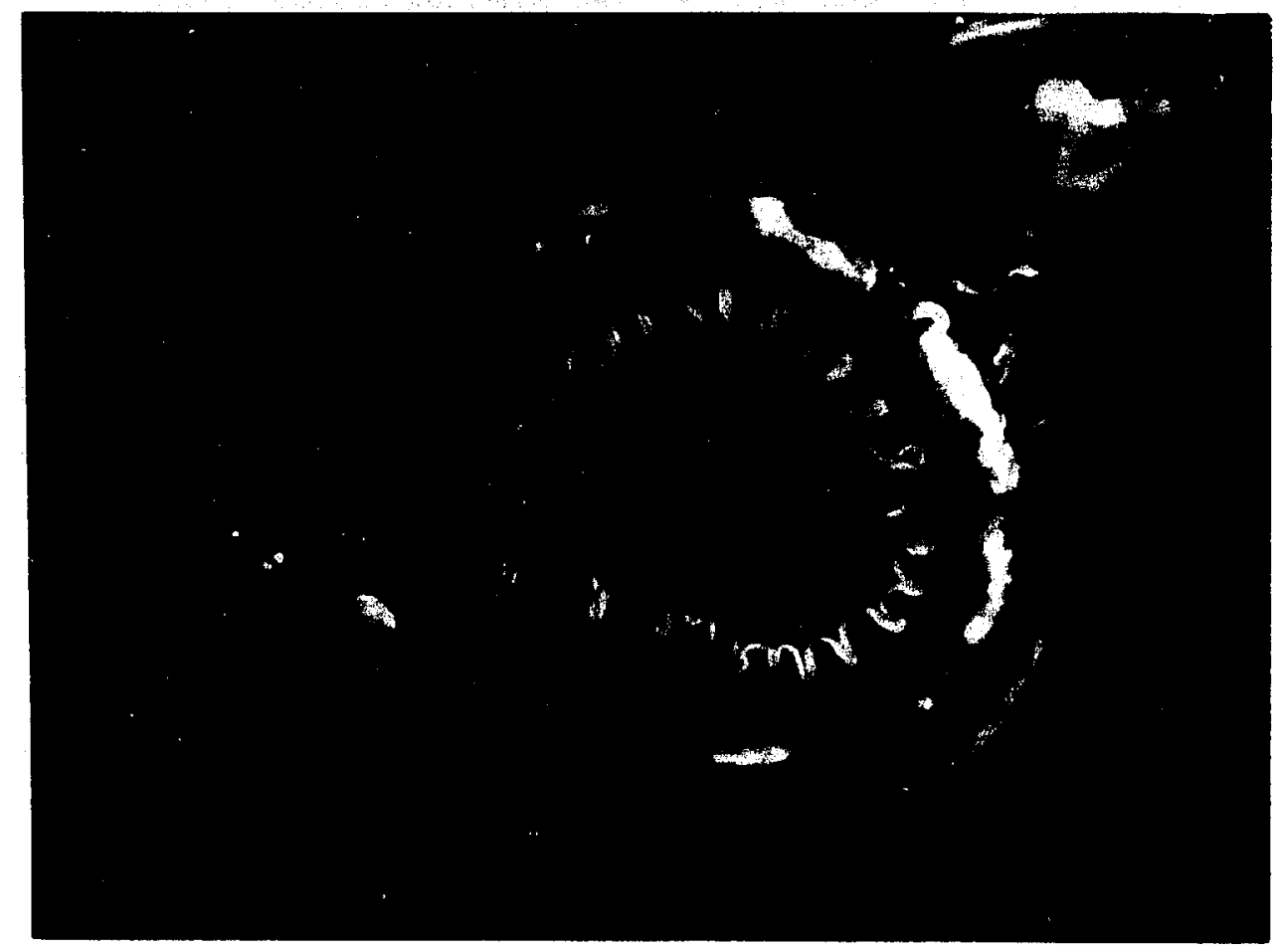


Plate IV. Histochemical studies of drug effects on central norepinephrine neurons.

Reserpine $+\alpha-m T+D L-t h r e 0-D O P S$ treated rat.

Presence of some fluorescence (green) in the tunica adventitia of the basilar artery indicates that $\mathrm{OL}-$ threo-DOPS produced some repletion of norepinephrine in anima1s, which received both reserpine and $\alpha-m$ T. Magnification X 160 . 


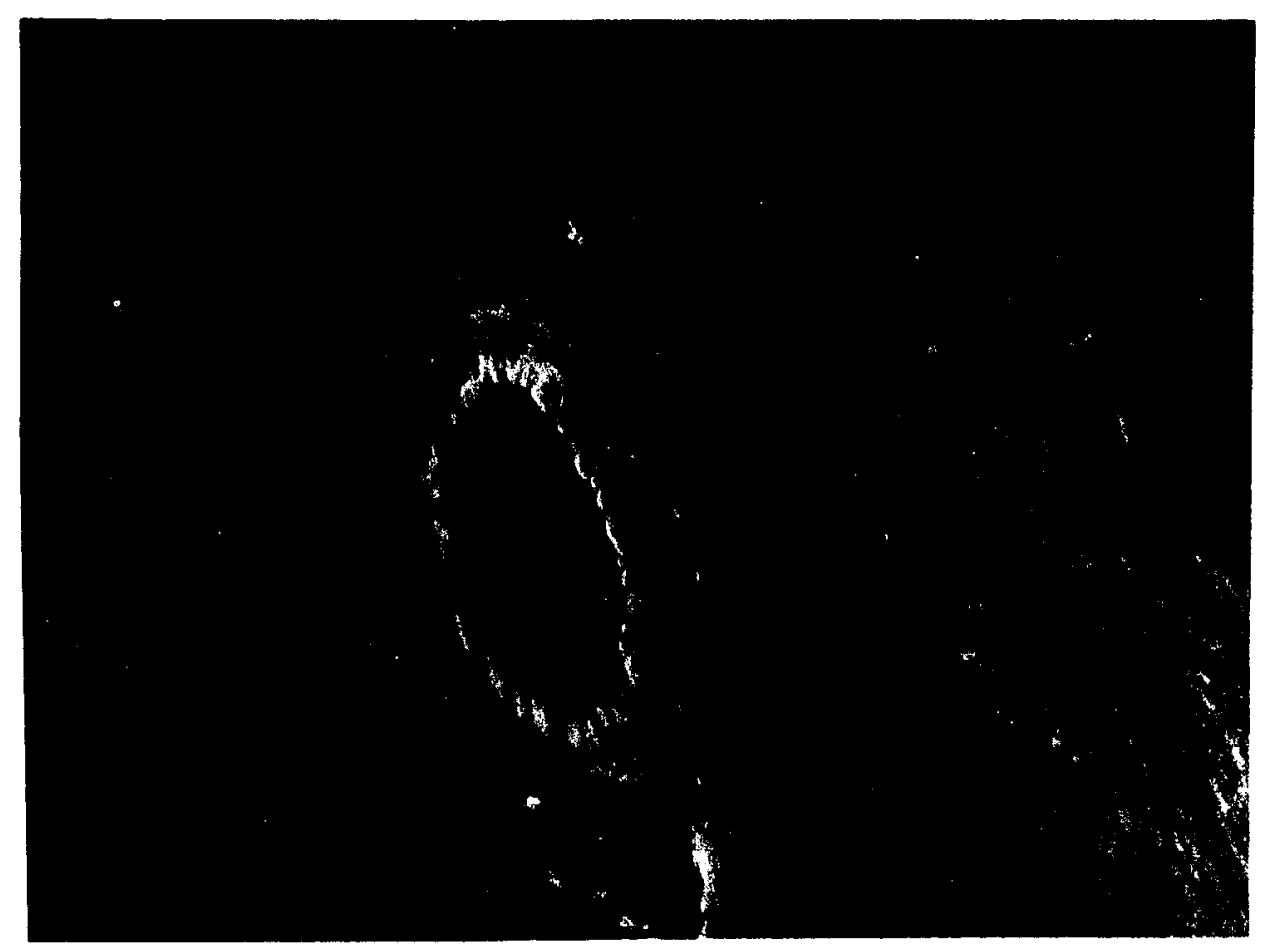


Plate V. Histochemical studies of drug effects on central norepinephrine neurons.

Top. Normal rat. Presence of a large collection of small to medium sized oval cells exhibiting medium to s.trong green fluorescence. This occurs within the area ventral is tegmenti and the zona compacta, ventrolateral to the medial Temniscus in the mesencephalon. These cells belong to the group of central catecholamine neurons classified as A10 by Dahlstrom and Fuxe (1964). Magnification $\times 160$.

Bottom. Reserpine treated rat. Same area as above. Almost total absence of green fluorescence indicates depletion of catecholamines. Magnification X 160 . 

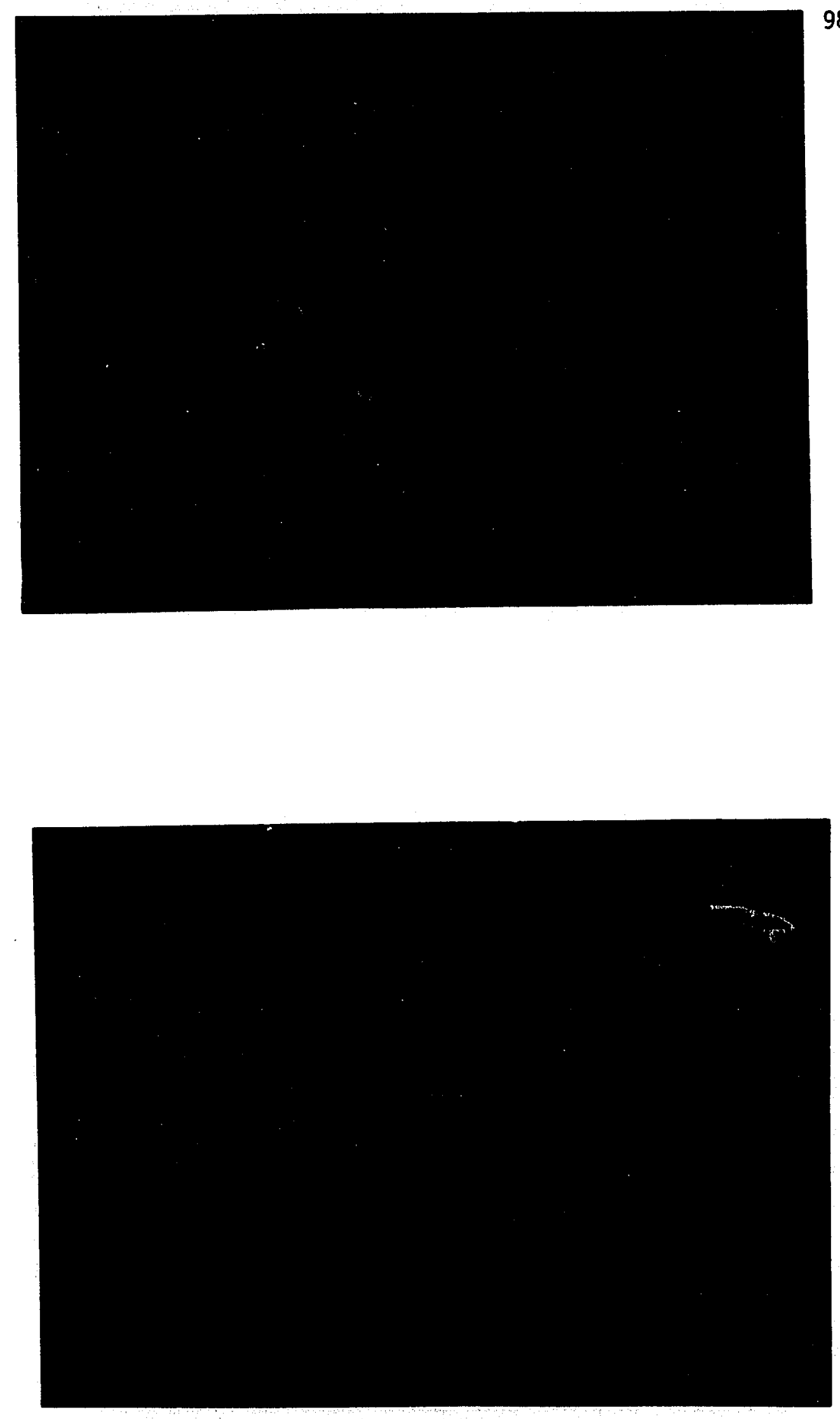
Plate VI. Histochemical studies of drug effects on central norepinephrine neurons.

Top. $\quad \alpha-m T$ treated rat. Same region as in Plate $V$. Absence of green fluorescence in this area indicates depletion of catecholamines by $\alpha-m T$. Magnification X 160 .

Bottom. Reserpine $+\alpha-m T+D L-$ threo-DOPS treated rat. Appearance of green fluorescence in the same area as Plate $V$, indicates repletion of catecholamines (1norepinephrine) by DL-threo-DOPS. Magnification $\times 160$. 

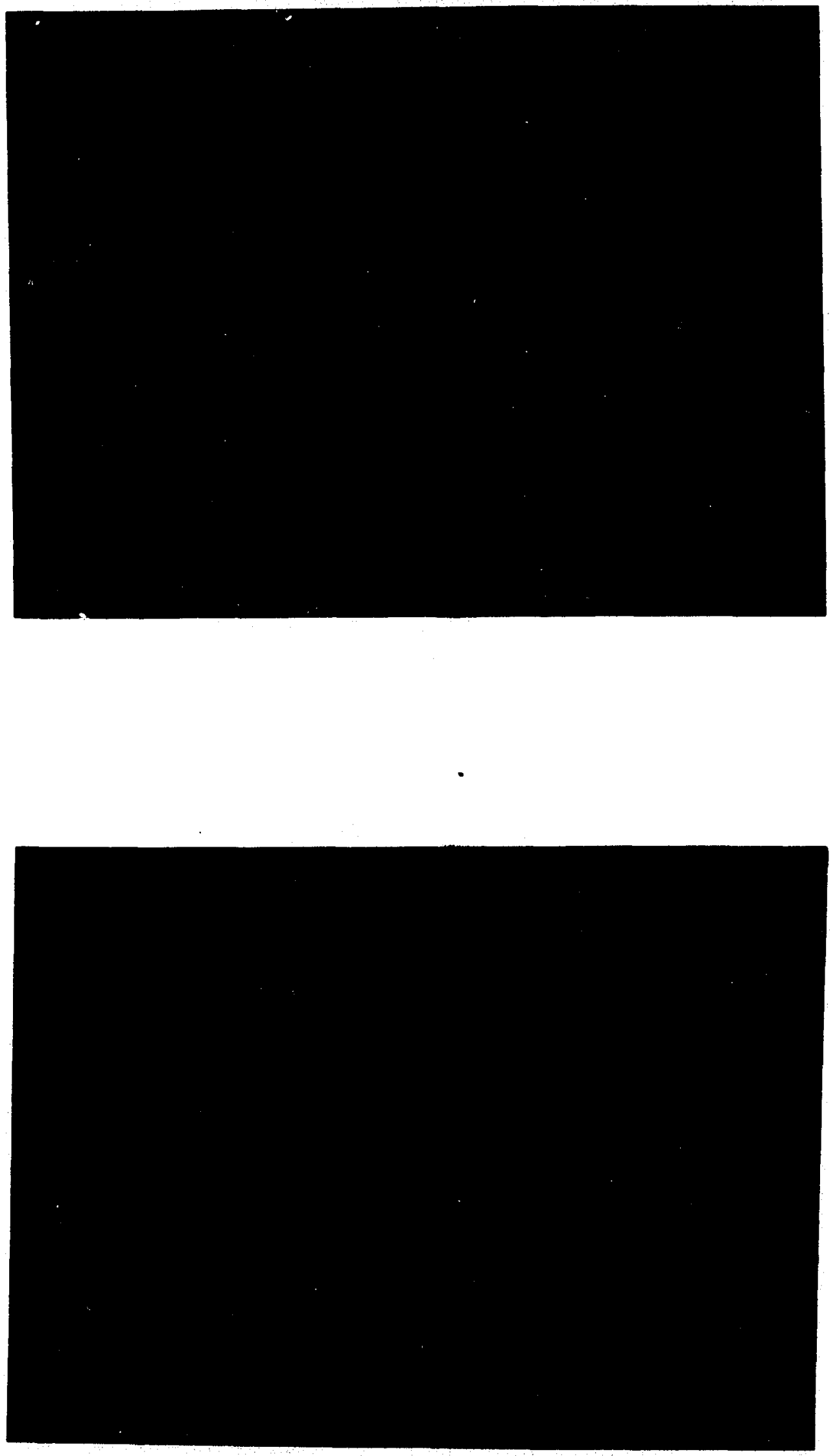
(indicated as white spots on Plate II:Top) surrounding the outer coat of the basilar artery. The lining of the Tumen also showed green fluorescence. According to Dahistrom and Fuxe (1964), both dopamine and norepinephrine condense with formaldehyde to form green fluorescent products, 6,7-dihydroxy-3,4-isoquinolines. Depletion of catecholamines $24 \mathrm{hr}$. after $2 \mathrm{mg} . / \mathrm{kg}$. reserpine was indeed achieved as indicated by the absence of fluorescence in this area (Plate Ir:Bottom). In this specimen, some residual fluorescence was sti1l observed, demonstrating incomplete catecholamine depletion.

The basilar artery exhibited intense green fluorescence in the tunica adventitia and the lumen lining, indicating no depletion of catecholamines at this site after $\alpha-m T$ (Plate III:Top). In fact, a greater amount of fluorescence seemed to exist in this specimen compared to the control (PIate II:Top). When reserpine was given in combination with $\alpha-m T$ there was almost complete disappearance of fluorescence in this area (Plate III:Bottom). Fluorescence still persisted in the lining of the lumen. Compared to the untreated specimen (Plate II:Top), there is significantly less fluorescence, indicating considerable depletion of catecholamines after reserpine and $\alpha-m T$ administration.

DL-threo-DOPS (200 mg./kg., $1 \mathrm{hr}$. before decapitation of the rat) was capable of restoring some green fluorescence in the outer and innermost layers of the basilar artery (Plate IV). In addition, the rest of the tissues demonstrated a nonspecific green fluorescence. This study indicates some repletion of norepinephrine with DL-threoDOPS in animals treated with both reserpine and $\alpha-m T$. The repletion 
was, however, incomplete since this specimen exhibited comparatively less fluorescence than the untreated sample (Plate II:Top).

The effects of these different pharmacological manipulations on norepinephrine content in central neurons (soma) are presented in Plates $V$ and VI. In the nomal rat (P1ate V:Top) there was present a large collection of small to medium size oyal cells of medium to strong green fluorescence within the area ventral is tegmenti and the zona compacta, ventrolateral to the medial lemniscus in the mesencephalon. According to the classification of Dahistrom and Fuxe (1964), these cells belong to group A10 of central catecholamine containing neurons. Reserpine treatment, prior to sacrifice, reduced the fluorescence of these cells almost completely (Plate V:Bottom). On closer examination some green fluorescent varicosities could be seen in the cytoplasm of these cells, but the nuclei seemed completely depleted of catecholamines.

The administration of $\alpha-\mathrm{mT}$ ( 3 doses of $50 \mathrm{mg} . / \mathrm{kg}$., given every $4 \mathrm{hr}$. and decapitation of the rats $12 \mathrm{hr}$. after the first dose) induced drastic reduction of green fluorescence in the cells in this anatomical area (PTate VI:Top). This indicates that central catecholamine neurons were indeed depleted by $\alpha-\mathrm{mT}$. Again, slight presence of green fluorescence could be detected in the cytoplasm of these cells, but the nuclei were devoid of any fluorescence.

When DL-threo-DOPS was given to catecholamine depleted animals the cells in this brain region demonstrated presence of green fluorescent varicosities. This was especially prominent in the cytoplasm of 
these cells (Plate VI:Bottom). In contrast, the specimens treated with either reserpine or $\alpha-m T$ per se showed almost complete absence of these varicosities (Plate V:Bottom; Plate VI:Top). The amount of fluorescent substance was much less compared to the untreated specimen (Plate V:Top), indicating that repletion of norepinephrine did occur under these experimental conditions, but that the restoration was incomplete.

A representative sample of this area in animals treated with both reserpine and $\alpha-m T$ was not presented since in this region no fluorescence was observed. A total1y dark photomicrograph was obtained. These data indicate that norepinephrine content in the brain did indeed vary with the various drug manipulations in this study.

The exact quantitative changes produced were evaluated by biochemical analysis of specific brain regions. These results are presented in Tables 3 and 4 . The doses and treatment intervals of drugs employed in this test were similar to those previously described. The assay procedure used was sensitive to $0.5 \mathrm{ng}$. of norepinephrine and was also specific for this compound. The wide confidence intervals seen may be due to the small number of animals per group (4) and to the sample-to-sample variability. As illustrated in Table 3, norepinephrine levels in the hypothalamus and the midbrain were significantly reduced to 27 and $32 \%$ of noma 1 , respectively after combined reserpine and $\alpha-m T$ treatment. Reduction of norepinephrine also occurred in the brain stem (54\%), but this was not significant compared to the control value. The norepinephrine contents in the hippocampus, cortex 
TABLE 3. Effects of Reserpine, $\alpha$-Methyl-p-Tyrosine and DL-threoDOPS on Norepinephrine Levels in Different Brain Areas in the Rat.

\begin{tabular}{|c|c|c|c|}
\hline Brain Area & Treatment & $\begin{array}{l}\text { Norepinephrine } \\
\text { (ng./gm.) }\end{array}$ & $\%$ Control \\
\hline \multirow[t]{4}{*}{ Brain Stem } & - & $127(79-128)^{a}$ & 100 \\
\hline & $\underset{\alpha-m T}{\text { Reserpine }}+$ & $57(10-123$ & 54 \\
\hline & $\begin{array}{l}\text { Reserpine }+ \\
\alpha-m T+D L-\text { threo- } \\
\text { DOPS }\end{array}$ & $91(57-125)$ & 60 \\
\hline & DL-threo-DOPS & $149(132-166)^{\mathrm{b}}$ & 117 \\
\hline \multirow[t]{4}{*}{ Hypothalamus } & - & $383(188-577)$ & 100 \\
\hline & $\underset{\alpha-m T}{\text { Reserpine }}+$ & $97(55-138)^{b}$ & 27 \\
\hline & $\begin{array}{l}\text { Reserpine }+ \\
\alpha-m T^{+}+D_{-} \text {-threo- } \\
\text { DOPS }\end{array}$ & $199(145-252)$ & 51 \\
\hline & DL-threo-DOPS & $508(415-600)$ & 133 \\
\hline \multirow[t]{4}{*}{ Midbrain } & - & $168(95-240)$ & 100 \\
\hline & $\underset{\alpha-m T}{\text { Reserpine }}+$ & $55(26-84)^{b}$ & 32 \\
\hline & $\begin{array}{l}\text { Reserpine }{ }^{+} \\
\alpha-\mathrm{mT}^{+}+\mathrm{DL}_{\text {-threo- }} \\
\text { DOPS }\end{array}$ & $104(80-127)$ & 62 \\
\hline & DL-threo-DOPS & $215(188-241)$ & 122 \\
\hline
\end{tabular}

a The figures within parentheses represent $95 \%$ confidence 7 imits. The total number of animals tested in each group was 4.

b Significantly different from control value $(P<0.05)$. 
TABLE 4. Effects of reserpine, $\alpha$-Methyl-p-Tyrosine and DL-threoDOPS on Norepinephrine Leyels in Different Brain Areas in the Rat.

\begin{tabular}{|c|c|c|c|}
\hline Brain Area & Treatment & $\begin{array}{l}\text { Norepinephrine } \\
\text { (ng./gm.) }\end{array}$ & $\%$ Control \\
\hline \multirow[t]{4}{*}{ Hippocampus } & - & $130(105-155)$ & 100 \\
\hline & $\underset{d-m T}{\text { Reserpine }+}$ & $130(0-260)$ & 100 \\
\hline & $\begin{array}{l}\text { Reserpine }{ }^{+} \\
\alpha-m T+L_{-} \text {-threo- } \\
\text { DOPS }\end{array}$ & $208(132-283)$ & 161 \\
\hline & DL-threo-DOPS & $299(236-363)^{\mathrm{b}}$ & 216 \\
\hline \multirow[t]{4}{*}{ Cortex } & - & $62(43-81)$ & 100 \\
\hline & $\begin{array}{l}\text { Reserpine }+ \\
\alpha-m T\end{array}$ & $57(0-114)$ & 92 \\
\hline & $\begin{array}{l}\text { Reserpine }+ \\
\alpha-m T+D L \text {-threo- } \\
\text { DOPS }\end{array}$ & $63(33-93)$ & 101 \\
\hline & DL-threo-DOPS & $142(117-166)^{b}$ & 229 \\
\hline & $+\cdots$ & $-\ldots$ & $\cdots$ \\
\hline \multirow[t]{4}{*}{ Corpus Striatum } & - & $112(58-166)$ & 100 \\
\hline & $\underset{\alpha-m T}{\text { Reserpine }}+$ & $129(26-232)$ & 115 \\
\hline & $\begin{array}{l}\text { Reserpine }+ \\
\alpha-m T+D L \text {-threo- } \\
\text { DOPS }\end{array}$ & $237(199-276)$ & 211 \\
\hline & DL-threo-DQPS & $215(98-332)$ & 192 \\
\hline
\end{tabular}

a The figures within parentheses represent $95 \%$ confidence 1 imits. The total number of animals tested in each group was 4.

b Significantly different from the control value. $(P<0.05)$ 
and the corpus striatum were not reduced following reserpine and $\alpha-\mathrm{mT}$ treatment (Table 4). A possible reason was the relatively low amounts of norepinephrine present in these areas. This made any further lowering of norepinephrine difficult to assess by the assay procedure employed.

Treatment with DL-threo-DOPS elevated cerebral norepinephrine concentrations in animals treated with reserpine and $\alpha-m$ T (Table 3 and 4). This was seen in all the regions analyzed, but the difference was significant at the $95 \%$ confidence level only in the hypothalamus. Here, the level was raised from 27 to $51 \%$ of the normal value. Considerable repletion (30\%) of norepinephrine occurred in the midbrain, although it was not statistically significant due to the small number of animals tested. These findings indicate that repletion of norepinephrine was indeed obtained by treatment with DL-threo-DOPS.

When DL-threo-DOPS was administered to naive rats, the norepinephrine concentration was raised in all the brain areas assayed (Table 3 and 4 ). This varied from a rise of $17 \%$ in the brain stem to $129 \%$ in the cortex. On closer examination, the brain stem, hypothalamus and the midbrain had increases of norepinephrine concentrations from 17 to $33 \%$, while the hippocampus, cortex and the corpus striatum values were increased from 90 to $129 \%$.

The above results indicate that depletion of brain norepinephrine by reserpine and $\alpha-m T$ per se or in combination increased the susceptibility of rats to seizures induced by electrical stimulation of the hippocampus. DL-threo-DOPS did produce some restoration of cerebral norepinephrine and its effect on seizure threshold in both naive and 
catecholamine depleted rats indicates a tendency of this compound to decrease the responsiveness of these animals to seizures. These findings are consistent with the hypothesis that a lack of norepinephrine enhances the susceptibility of an animal to seizures.

It is well known that norepinephrine acts predominently at alpha adrenergic sites in the periphery. The possibility that norepinephrine might be exerting its influence on seizure threshold via activity at corresponding central alpha adrenergic receptors was investigated in a study using compounds with alpha adrenergic blocking actions. Towards this end, the effects of phentolamine and phenoxybenzamine on seizure threshold were evaluated. The doses of phentolamine employed were selected on the basis of pilot neurotoxicity studies with this substance (see GENERAL PROCEDURE for data). At toxic doses phentolamine induced rhythmic jaw movements and reduced exploratory behavior. The effect of this drug on seizure threshold is presented in Figure 6 . Doses ranging from 0.25 to $0.75 \times 10^{-1}$ mMoles $/ \mathrm{kg}$., did not alter seizure threshold. These doses ranged from $1 / 4$ to $3 / 4$ the toxic dose 50 . Rats which received $0.75 \times 10^{-1}$ mMoles $/ \mathrm{kg}$. phentolamine had abnormal posture. When placed in the test chamber and given subthreshold shocks, these animals lay on one side of their body with their hind limbs exhibiting slow kicking movements. At this dose, one animal was sedated and displayed no exploratory behavior. The drug changed neither the sensitivity of the animals to seizures nor did it intensify the severity of the seizures. At threshold current, only rhythmìc jaw movements or fore $1 \mathrm{imb}$ clonus were elicited. 
Figure 6. Effect of phentolamine on seizure threshold. Threshold Ratio was determined by dividing the drug seizure threshold or the control seizure threshold by the stable seizure threshold. The vertical bars denote $95 \%$ confidence limits. $\Delta$, phentolamine; 0 , distilled water. 


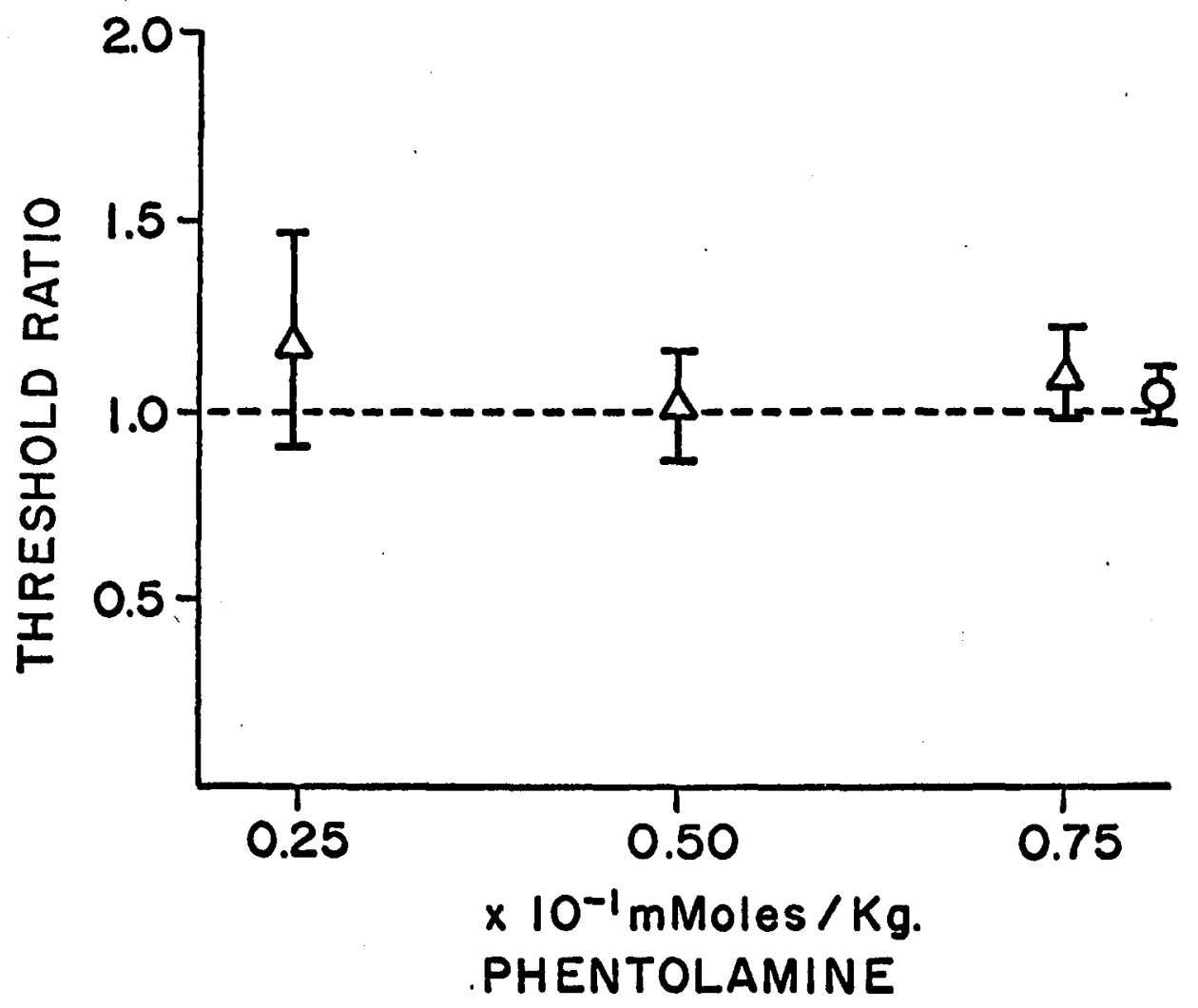


The doses of phenoxybenzamine investigated were likewise based on neurotoxicity studies (GENERAL PROCEDURE). At toxic doses this compound induced central nervous system depression and rhythmic jaw movements. In contrast to saline treated rats, these animals showed little grooming behavior. Phenoxybenzamine had a prolonged toxic action as indicated by 2 deaths from a test group of 6 rats treated with 3.0 $\times 10^{-1} \mathrm{mMoles} / \mathrm{kg}$, $24 \mathrm{hr}$. after injection. The data collected in a dose-response study of this compound on seizure threshold are presented in Figure 7. Doses less than $1 / 4$ the toxic dose 50 were examined. Only $0.375 \times 10^{-1} \mathrm{mMoles} / \mathrm{kg}$. produced slight depression (20\%) of seizure threshold; the other doses were ineffective. The drug vehicle, $10 \%$ propylene glycol in saline did not al ter seizure threshold. Gross observations of these test animals indicated no aberrant behavior; normal exploratory motions being displayed by drug treated animals when placed in the test chamber.

These findings show that, of the 2 alpha adrenergic blocking drugs investigated, only phenoxybenzamine produces a decrease in seizure threshold.

Besides its activity on alpha adrenergic sites, norepinephrine is also known to act on beta adrenergic receptors. The possible involvement of beta adrenergic sites in the manifestation of seizures was investigated in a study of the effects of several compounds known to block beta adrenergic activities in the periphery. Propranolo1, pronetha101, sotalol and $D(-2$ INPEA were examined, In addition, $L(+)$ INPEA, known to have comparatively weak blocking action on beta adrenergic re- 
Figure 7. Effect of phenoxybenzamine on seizure threshold. Threshold Ratio was determined by dividing the drug seizure threshold or the control seizure threshold by the stable seizure threshold. The vertical bars denote $95 \%$ confidence limits. $\Delta$, phenoxybenzamine; $0,10 \%$ propylene glycol in saline. 


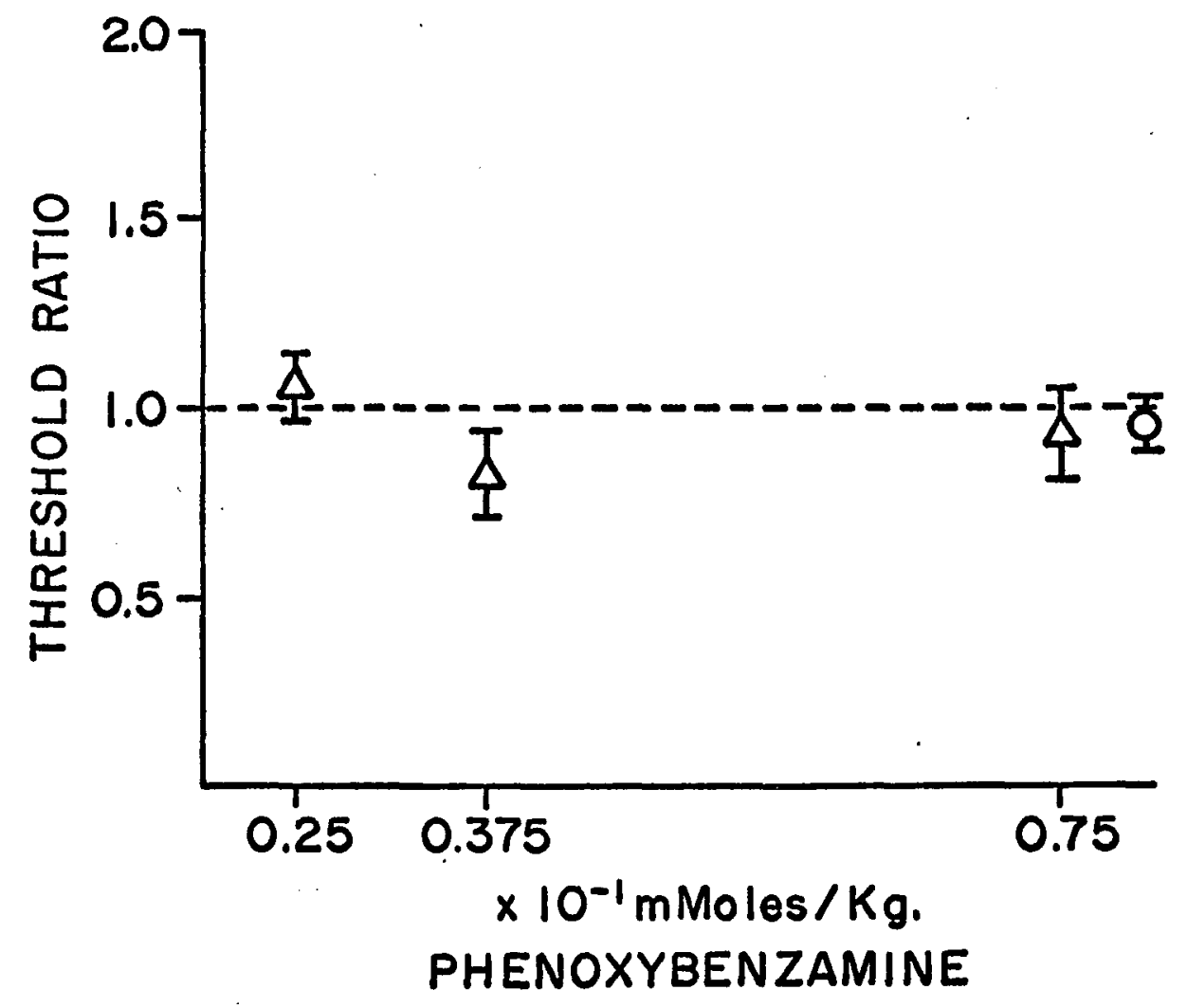


ceptors (Almirante and Murmann, 1966; Patil, 1968) was also studied for purposes of comparison with its $D(-)$ isomer. Again, the doses investigated were selected on the basis of neurotoxicity tests (GENERAL PROCEDURE). These tests show that with toxic doses of propranolol, animals were centrally depressed, ataxic and failed to perform the rolling-bar task. At $2 \times 10^{-1}$ mMoles $/ \mathrm{kg} ., 1$ rat became hypersensitive. A light touch on its feet was sufficient to induce an extensor reflex. Doses of less than $1 / 2$ the toxic dose 50 were employed in seizure threshold studies. The data are presented in Figure 8. No change of seizure susceptibility was observed in animals receiving $0.25 \times 10^{-1}$ mMoles $/ \mathrm{kg}$. of the drug. However, higher doses produced significant elevations of seizure threshold. An increase of about $98 \%$ was seen with $0.375 \times 10^{-1} \mathrm{mMoles} / \mathrm{kg}$., while an augmentation greater than $275 \%$ followed $0.5 \times 10^{-1} \mathrm{mMoles} / \mathrm{kg}$. At the highest dose investigated, several rats did not exhibit seizures even with the administration of the maximum stimulus. Such "protected" animals were assigned a threshold current of $3,000 \mathrm{UA}$ for statistical purposes. Therefore, apparent dose-response relationship may not be real, since the actual threshold currents in "protected" rats were not determined. Again, the control studies revealed no alteration of seizure threshold.

From neurotoxicity studies (GENERAL PROCEDURE), pronetha101 was observed to interfere with the ability of rats to hold onto the rollingbar. With a dose of $2 \times 10^{-1} \mathrm{mMoles} / \mathrm{kg} ., 1$ out of 6 animals displayed short-lived convulsions after 3 falls from the rolling bar, subsequent to which it was hyperactive. In the seizure threshold tests, doses 
Figure 8. Effect of propranolol on seizure threshold.

Threshold Ratio was determined by dividing the drug seizure threshold or the control seizure threshold by the stable seizure threshold. The vertical bars denote $95 \%$ confidence 1 imits. $\triangle$, propranolol; 0 , saline. At $0.5 \times 10^{-1}$ mMoles/ kg., several animals did not exhibit seizures on exposure to the maximum current and were assigned seizure threshold of 3,000 uA for statistical computations. 


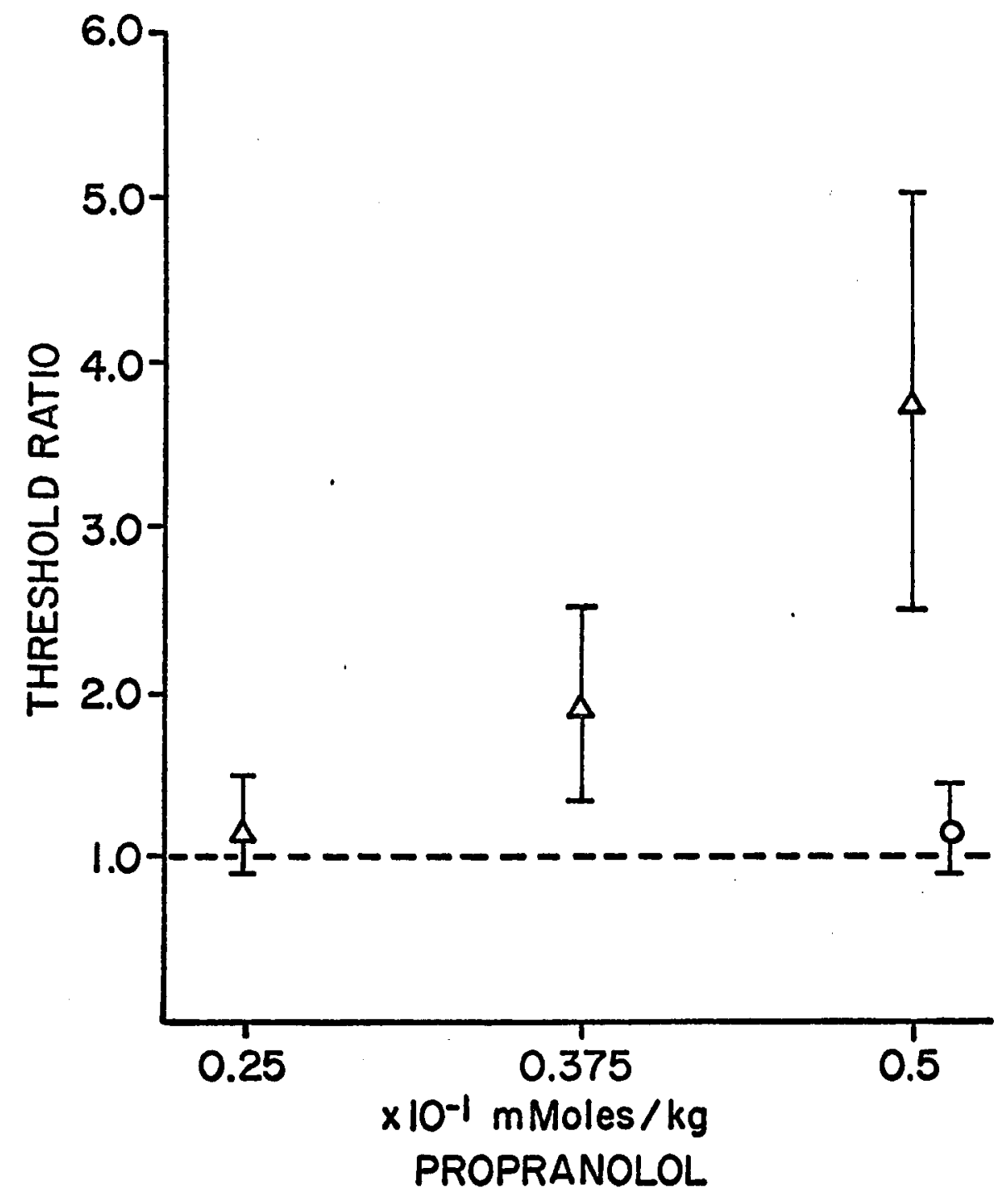


of less than toxic dose 50 were investigated. No observed abnormal behavior was exhibited by any of these animals. The effect of several doses of pronethatol on seizure threshold is reported in Figure 9. With doses less than $1 / 2$ toxic dose 50 , the compound increased seizure threshold significantly. A 50\% elevation was observed with $0.5 \times 10^{-1} \mathrm{mMoles} / \mathrm{kg}$. Seizure thresholds were unal tered in animals injected with doses higher than $0.5 \times 10^{-1} \mathrm{mMoles} / \mathrm{kg}$. It is possible that at these higher doses the central excitation produced by this drug may have confounded its anticonvulsant property.

As indicated by the neurotoxicity studies (GENERAL PROCEDURE), sotalol (MJ1999) is relatively nontoxic. All animals were able to perform the rolling-bar test, even with the administration of $3 \times 10^{-1}$ mMoles $/ \mathrm{kg}$. sota101. With this dose gross observations revealed that these animals lacked exploratory behavior. Some central depression was also seen. In addition, 2 out of 5 animals displayed occasional jaw movements resembling eating. The responsiveness of rats to seizures after sotalol is presented in Figure 10. The drug did not alter seizure threshold with doses ranging from 0.5 to $2.0 \times 10^{-1} \mathrm{mMoles} / \mathrm{kg}$. The highest dose investigated was about 5 times the effective dose of propranolo1.

Neurotoxicity studies with $D(-)$ INPEA show that this compound can induce minimal and maximal seizures at toxic doses (GENERAL PROCEDURE). Following $2 \times 10^{-1} \mathrm{mMoles} / \mathrm{kg}$., 3 out of 6 animals were hyperactive. Similar symptoms were observed in animals receiving toxic doses of the $L(+)$ isomer. In seizure threshold evaluations, doses less than the 
Figure 9. Effect of pronethalol on seizure threshold.

Threshold Ratio was determined by dividing the drug seizure threshold or the control seizure threshold by the stable seizure threshold. The vertical bars denote 95\% confidence limits. $\triangle$,pronethalol; 0 , saline. 


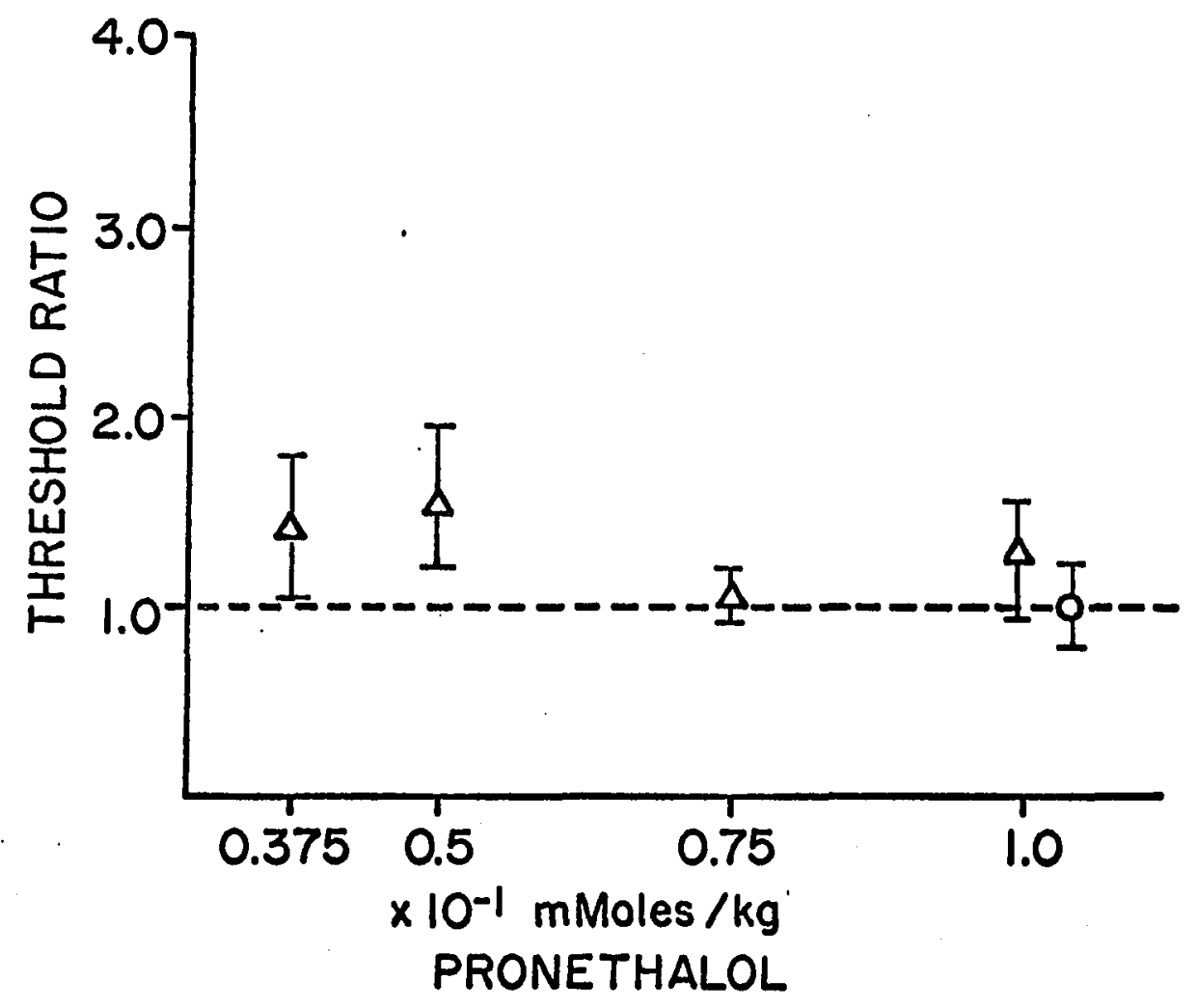


Figure 10. Effect of sotalol (MJ1999) on seizure threshold. Threshold Ratio was determined by dividing the drug seizure threshold or the control seizure threshold by the stable seizure threshold. The vertical bars denote $95 \%$ confidence limits. $\triangle$, sotalo1; $O$, saline. 


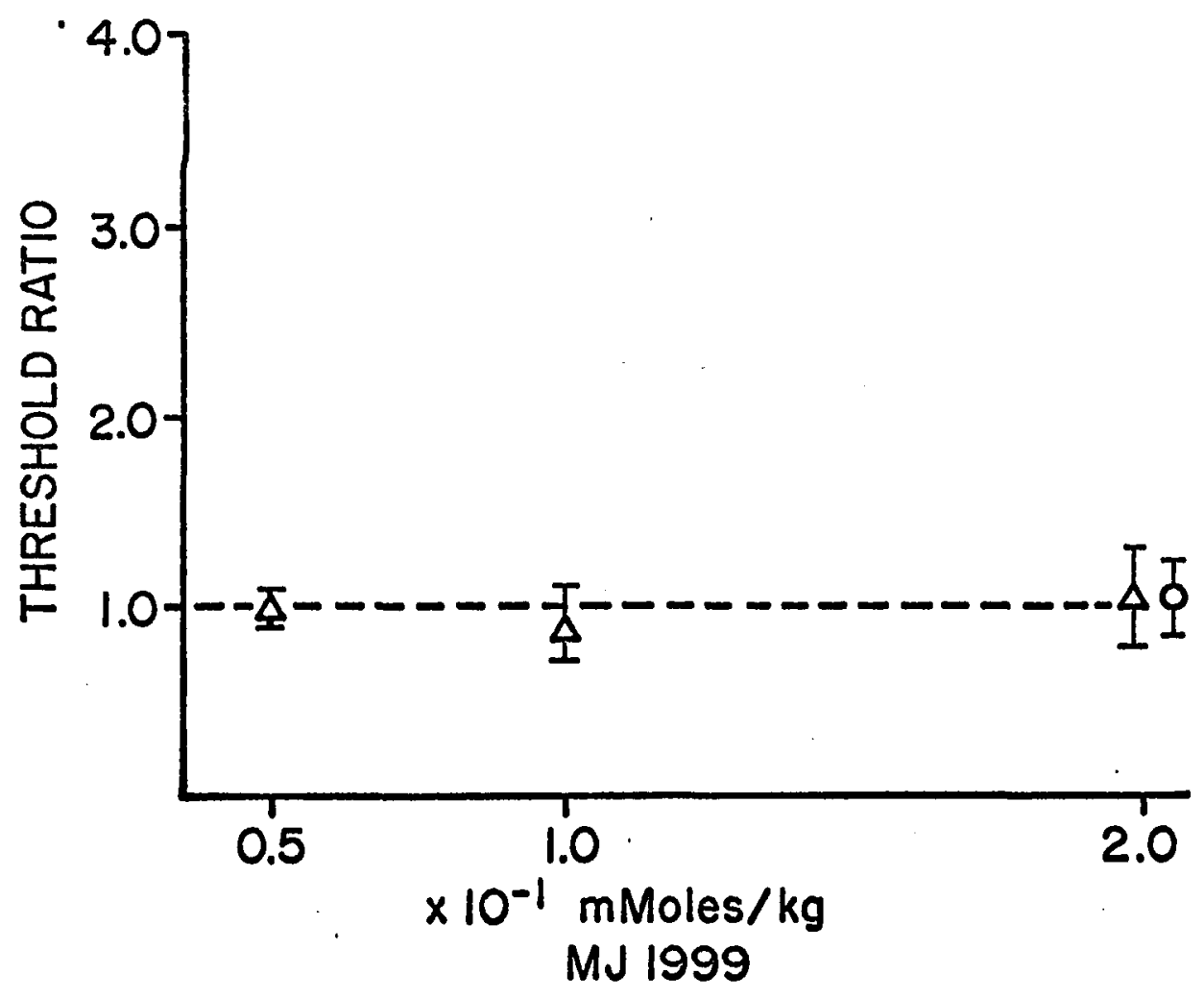


Figure 11. Effect of INPEA on seizure threshold. Threshold Ratio was determined by dividing the drug seizure threshold or the control seizure threshold by the stable seizure threshold. The vertical bars denote 95\% confidence limits. $\triangle, D(-)$ INPEA; $\boldsymbol{\Delta}$, $L(+)$ INPEA; $O$, saline. 


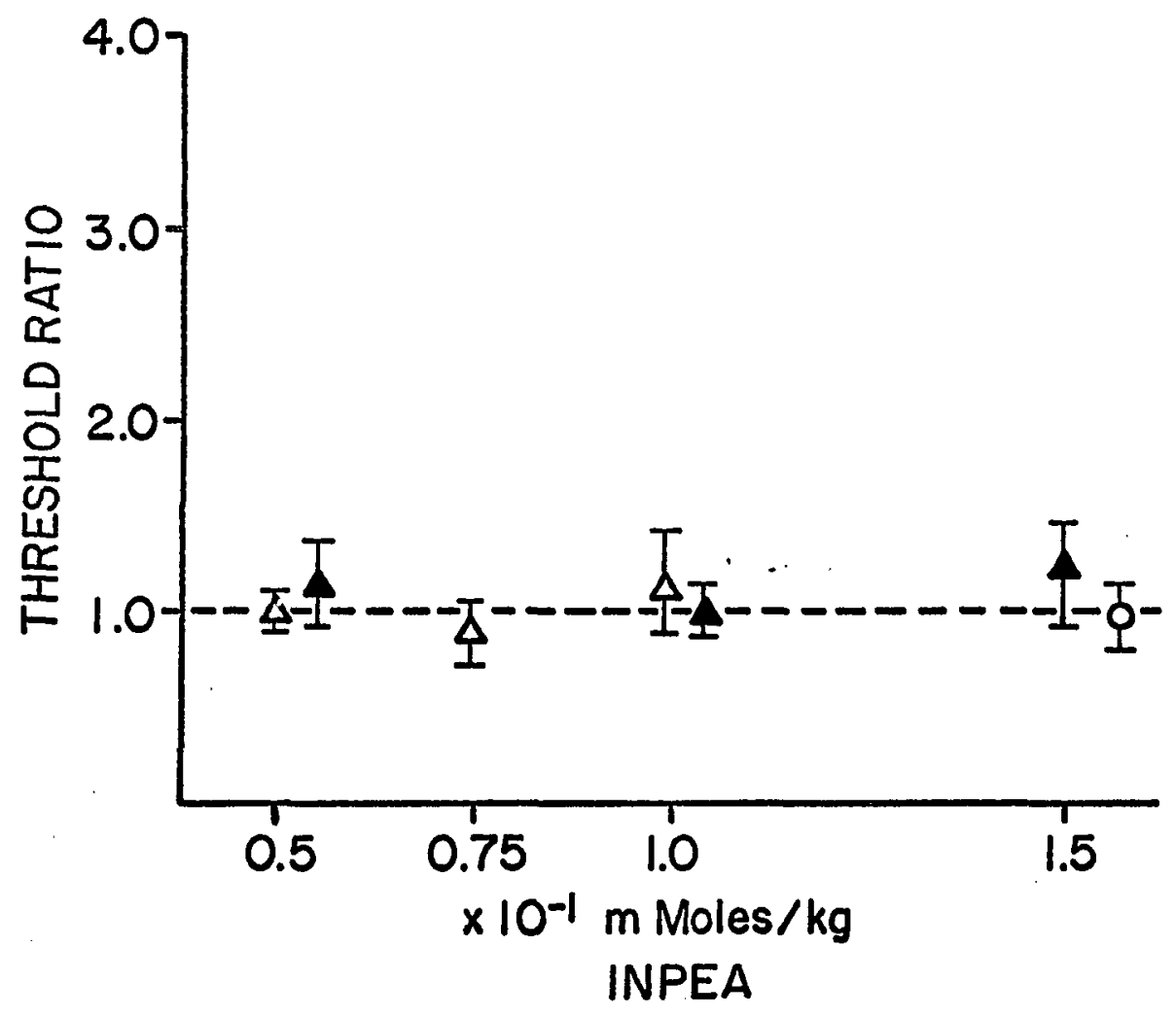


toxic dose 50 were investigated. Both $D(-)$ and $L(+)$ INPEA produced no change in seizure susceptibility at doses from 0.5 to $1.5 \times 10^{-1}$ mMoles $/ \mathrm{kg}$. (Figure 11). With $0.75 \times 10^{-1} \mathrm{mMoles} / \mathrm{kg} ., D(-)$ INPEA, 1 of the test animals exhibited maximal seizures with hind 1 imb clonus, but no tonic extension. This was different from the control studies, where only minimal seizures with facial and fore 1 imb clonus were present.

These studies with 5 compounds which possess varying degrees of blockade at beta adrenergic receptors show that only propranolol and pronethalol raise the thresholds of rats to seizures induced by electrical stimulation of the hippocampus.

D. Discussion

As illustrated in Figure 5, reserpine reduced the threshold of rats to electrically-induced hippocampal seizures. The severity of seizures was enhanced as illustrated by the presence of maximal seizures at threshold current and the occurrence of death following convulsions. These findings agree with those reported by other workers who employed different methods. For example, reserpine shortens the latency of susceptible animals to audiogenic seizures while rendering resistant animals sensitive to sound-induced seizures (Cooke et al., 1960, Cooke, 1961). As discussed in the Introduction, the incidence of pentylenetetrazol-induced seizures in animals and their subsequent death are both increased by reserpine. Thresholds to several types of electricallyproduced seizures are also lowered after reserpine. 
As shown in histochemical studies (P1ate II, BOTTOM; Plate V, BOTTOM), reserpine lowered norepinephrine concentrations in rat brain. This effect could be responsible for the ability of reserpine to enhance seizure susceptibility in the present study. However, since reserpine also reduces dopamine and serotonin levels, functional changes following its treatment can be due to a deficiency of any one, or combinations of these amines (Zaimis, 1961).

Reserpine alters monoamine concentrations in vivo via different mechanisms. Its primary action is to inhibit the specific $\mathrm{Mg}^{++}$-ATPdependent uptake mechanism of storage granules, leading to depletion of biogenic amines (Bertler, 1961; Dahlstrom, 1965; Glowinski et al., 1966; Anden et al., 1969). Support for such a mechanism was furnished from studies which demonstrated the ability of reserpine to block intracellular localization of biogenic amines formed from their precursors in the adrenal medulla (Bertler et al., 1960; Carlsson et al., 1962; 1963; Kirshner, 1962) and in the nerve endings (EuTer and Hitlarp, 1956; Euler, 1958; Euler and Lishajko, 1961; 1963; 1964). Further evidence was obtained from experiments which showed that the adrenergic transmitter taken up subsequent to reserpine administration cannot be retained unless protected from intraneuronal deamination (Hillarp and Malmfors, 1964; Malmfors, 1965).

Reserpine also reduces the accumulation of intraventricularly injected H3-catecholamines in the brain (Glowinski and Iversen, 1966).

Histochemical evidence shows that reserpine releases norepinephrine from all parts of the neuron (Carlsson et al., 1962; Dahlstrom and Fuxe, 1964). However, the onset and amount of amine depletion vary in dif- 
ferent regions (Carlsson et al., 1962; Dahlstrom and Fuxe, 1965). For example, norepinephrine is more sensitive than dopamine to reserpine action in the whole brain (Bertler, 1961). In a more detailed study, (Anden, 1967) specific brain areas were examined for norepinephrine, dopamine and serotonin concentrations before and after reserpine administration. Norepinephrine was depleted to a greater degree than serotonin in the neopallium, spinal cord and the thalamus. Depletion of norepinephrine in the neopallium also occurred to a greater extent compared to reduction of dopamine in the corpus striatum. In addition, reserpine influenced the norepinephrine in the neopallium to a greater extent than in the hypothalamus or in the nucleus interstitial is stria terminalis. Regional variation in the responsiveness of dopamine to reserpine depletion was demonstrated by greater sensitivity in the tuberculum olfactorium and the nucleus accumbens compared to the corpus striatum. In addition, the reduction of norepinephrine in the heart was more pronounced than in the neopallium.

Following reserpine administration, functional changes appear to be better correlated with the recovery of neuronal accumulation of H3norepinephrine rather than with the absolute brain content of norepinephrine or other amines (Everett and Wiegand, 1961; Haggendal and Lindqvist, 1963; 1964; Lundborg, 1963; Carlsson, 1965; Sedva11 and Thorson, 1965; Glowinski et al., 1966; Lundborg and Stitze1, 1968).

The time required for total recoyery of norepinephrine levels in different tissues of rat and rabbit after a large dose of reserpine is about the same as the life span of the storage granules of both species 
(Dahistrom and Haggendal, 1966). Furthermore, the recovery is almost linear, indicating that reserpine action on storage mechanisms is largely irreversible - recovery probably occurs through a steady state down-transport of newly formed amine granules.

After reserpine treatment, the endogenous norepinephrine concentrations take several weeks to return to normal levels (Bertier, 1961). According to Alpers and Shore (1969), the presence of small amounts of reserpine probably maintain this persistent depletion of norepinephrine through saturation of the amine carrier.

Conflicting opinions exist with regard to the ability of reserpine to inhibit the biosynthesis of norepinephrine. On the basis of their metabolic studies with intraventricularly administered Cl4-tyrosine and H3-dopamine in normal and reserpinized rats, Glowinski and co-workers (Glowinski and Iversen, 1966; Glowinski et al., 1966) suggested that reserpine does not interfere with catecholamine synthesis. On the other hand, some workers have presented data which support their contention that reserpine inhibits norepinephrine synthesis by limiting the access of dopamine (extragranular) to dopamine- $\beta$-oxidase, which is intragranular in location (Kirshner, 1962; Kirshner et al., 1963; Rutledge and Weiner, 1967; Stjarne et al., 1967; Roth and Stone, 1968; Jonason and Rutledge, 1969).

In both the brain and the periphery, reserpine-resistent pools of norepinephrine are present (Haggendal and Lindqvist, 1964; Kopin, 1964; Sedva11, 1964; Fischer et al., 1965; Iversen et al.; 1965). This is also i1lustrated from H3-norepinephrine accumulation studies where a 
residual amount of H3-norepinephrine is not released by an additional dose of reserpine (Glowinski and Axelrod, 1965; Glowinski et al., 1966). There is also evidence demonstrating the possible existence of a reversible reserpine effect on small functional pools of the granules (Shore, 1960; Jonason et al., 1964).

The above survey indicates that depletion of biogenic amines by reserpine can occur from inhibition of amine uptake and possibly from blockade of biosynthesis. However, small reserpine-resistant pools of norepinephrine remain. These can maintain a certain degree of physiological function and can possibly contribute to the present observation that seizure threshold was depressed by $50 \%$ only after the drug.

In the current study, $\alpha-m T$ lowered seizure threshold in naive and reserpinized rats (Figure 5). Combined treatment with reserpine and $\alpha-m T$ increased the convulsive responsiveness of rats to a greater extent compared to treatment with $\alpha-\mathrm{mT}$ per se. However, the amount of seizure threshold depression was no greater than in those animals injected with reserpine per se. This could result from 2 possible effects. First, the level of seizure excitability could be sufficiently maintained by the small amount of norepinephrine remaining in vivo after treatment with reserpine and $\alpha-m T$. As indicated in Table 3, the hypothatamus and the midbrain retained about $30 \%$ of the normal norepinephrine concentrations following reserpine and $\alpha-m T$. Second, inherent 1 imitations of the experimental design and technique made evaluation of seizure threshold depression greater than $50 \%$ difficult 
and extremely variable. This difficulty probably masks the detection of seizure threshold differences between groups with very low epinephrine levels.

By itself, $\alpha-m T$ did not enhance the severity of seizures at threshold current. In reserpinized animals treated with $\alpha-m T$, marked increase in seizure severity occurred. At threshold current, maximal seizures were elicited in the experimental rats. Following the convulsive episodes, all animals died. Since a lowered incidence of lethality occurred and a lesser number of animals exhibited maximal seizures after reserpine per se, it may be suggested that in such animals, inhibition of norepinephrine synthesis by $\alpha-m T$ probably removes the small functional pools of norepinephrine and increases seizure severity.

The additional finding that cumulative doses of $150 \mathrm{mg} / \mathrm{kg} . \quad \alpha-\mathrm{mT}$ lowered seizure threshold to a greater extent than $240 \mathrm{mg} . / \mathrm{kg}$., suggests that nonspecific toxicity at the higher dose (as indicated by hypothermia and profound central depression) probably confounds its effects on seizure threshold. The central depression following $\alpha-m T$ was potentiated in reserpinized rats. Similar findings have also been reported by other workers (Rech et al., 1966b; Moore and Rech, 1967).

These data are consistent with those obtained by other workers (Rudzik and Mennear, 1966a; 1966b; Chen et al., 1968a). As shown in the introduction to this section, $\alpha-m$ T increases the susceptibility of animals to maximal electroshock seizures and hind 1 imb extensor seizures. 
Alpha-methyl-p-tyrosine reduces catecholamine concentrations in vivo through an action on tyrosine hydroxylase in the overall production of norepinephrine. The conversion of tyrosine to dopa by tyrosine hydroxylase is the rate-limiting step in the biosynthesis of norepinephrine. The enzyme functions in the presence of a tetrahydropteridine cofactor, $\mathrm{Fe}^{++}$and oxygen (Udenfriend, 1966). The activity of this enzyme may be inhibited by substrate competition (Udenfriend et al., 1965; Udenfriend, 1966; McGeer et al., 1967), competition with the pteridine cofactor (Udenfriend et al., 1965; Goldstein et al., 1967), or via iron chelation (Taylor et al., 1969). At present, the best known tyrosine hydroxylase inhibitor is $\alpha$-methyl-p-tyrosine $(\alpha-m T)$, which inhibits the enzyme in vivo. This leads to a reduction in brain norepinephrine and dopamine (Spector et al., 1965; Rech et al., 1966b; Udenfriend, 1966). The mechanism of action is substrate inhibition (Udenfriend, 1966).

Since tyrosine hydroxylase activity is rate-limiting, the most effective way to block norepinephrine synthes is is to block this enzyme activity. Udenfriend (1966) compared inhibitors of tyrosine hydroxylase, dopa decarboxylase and dopamine- $\beta$-oxidase with respect to conversion of C14-tyrosine and H3-dopa to norepinephrine in vivo. He found that tyrosine hydroxylase inhibitors, $\alpha-m T$ and $\alpha$-methylphenylalanine were most effective. In addition, lowering of norepinephrine levels in various tissues of the guinea pig by $\alpha-m T$ was directly related to the degree of tyrosine hydroxylase inhilitition. 
Following $\alpha-m T$, brain catecholamines are decreased to a greater extent than peripheral catecholamines (Porter et al., 1966). Biochemical studies indicate that norepinephrine and dopamine are reduced to about the same extent in various forebrain regions, but only slightly in the heart (Anden, 1967). This is just the reverse with reserpine treatment, where the heart is more sensitive to catecholamine depletion than the forebrain. Histochemically, it has been demonstrated that $\alpha-m T$ produces uniform depletion of catecholamines in various forebrain areas (Anden et al., 1966a). In the hypothalamus, norepinephrine in various nuclei is depleted to about the same extent, unlike what is seen following reserpine administration, where large differences occur. Along these lines it is interesting to note that in the present study, following $\alpha-m T$ treatment, norepinephrine was depleted in brain cells, but not in norepinephrine neurons surrounding cerebral blood vessels (P1ates VI:TOP; III:TOP). In fact the amount of fluorescence was intensified in these latter areas. This is perhaps related to a feedback mechanism for the control of synaptic activity (Anden et al., 1969). Since depletion occurs at somas, the impulse flow to nerve terminals is reduced, bringing into play a possible feedback mechanism, which could accelerate norepinephrine turnover. Such an explanation is supported by the finding that $\alpha-m T$ depends on nerve impulses for its monoamine depleting action (Anden et al., 1966a; 1966b; 1967).

Unlike reserpine, $\alpha-m$ T causes a specific fall in brain dopamine and norepinephrine without affecting brain serotonin levels (Spector et al.; 1965; Weissman and Koe, 1965; Brodie et al., 1966; McGeer et al, 
1967). The storage of catecholamines is also unaffected by $\alpha-m T$ (Corrodi et al., 1966). Due to the presence of large "reserve" stores of catecholamines within the neuron, a latent period exists before catecholamine stores are severely depleted following inhibition of synthesis (Rech et al., 1966a; 1966b). With high doses of $\alpha-m T$, nephrotoxicity occurs (Hanson, 1965; Moore, 1966). In order to overcome this problem, $\alpha-m T$ is usually given in cumulative doses (Rech et al., 1966a; 1966b; Moore et al., 1967), and administered in combination with reserpine (Rech et al., 1967). Such a schedule effectively destroys the large "storage" pools of catecholamines with reserpine and blocks synthesis with $\alpha-m T$, thus compromizing the smaller, functionally active pools, which may be maintained by newly synthetized amines.

The histochemical (Plates II to VI) and biochemical (Tables 3 and 4) data confirm that reserpine or $\alpha$-mT administered per se or in combination produced significant depletion of brain norepinephrine. Since these drugs lowered seizure susceptibility in the test rats, the above data implicates a norepinephrine influence on the susceptibility of rats to seizures induced by hippocampal stimulation.

Although the above data indicate increased seizure susceptibility following reserpine and $\alpha-m T$ treatment, no conclusive deduction can be made with regard to the specific neurotransmitter(s) responsible for the observed effect. However, by selective repletion of 1-norepinephrine through decarboxylation of exogenously administered DL-threo-DOPS in animals treated with reserpine and $\alpha-m T$, the selective effect of norepinephrine on seizure threshold can be evaluated. Under the experi- 
mental conditions employed, 20 to $30 \%$ repletion of 1-norepinephrine was achieved in the hypothalamus and the midbrain (Table 3l. In these animals, seizure excitability was also reduced (see Figure 5). A1though the change was not very impressive, it was definitely in the direction of elevating seizure threshold. It would be interesting to see whether a more pronounced effect could be produced with higher doses of the amino acid precursor.

A7 though DL-threo-DOPS per se increased markedly, the norepinephrine concentrations in the hippocampus, cortex and the corpus striatum (Table 4), it did not produce a significant elevation of seizure threshold (Figure 5). Such an observation resembles that reported for the effects of 1-dopa on seizure susceptibility. As discussed in the Introduction, 1-dopa per se generally does not alter seizure excitability, but the drug effectively lowers seizure susceptibility in the presence of a monoamine oxidase inhibitor. Whether DL-threo-DOPS would have seizure threshold elevating effects in the presence of monoamine oxidase inhibition must await further experimentation.

In naive animals, DL-threo-DOPS produced no significant elevation of norepinephrine in the hypothalamus or the midbrain (Table 3 ). This may account for its lack of effect on seizure threshold (Figure 5).

In contrast to the findings of Carlsson (1964), DL-threo-DOPS produced central depression in naive rats in this study. It did not a)ter the sedation seen in reserpinized animals, given $\alpha-m T$. Carlsson (1964) reported presence of central excitation in mice following DL-threo-DOPS. Furthermore, an "awakening" effect by the compound 
in reserpine treated animals was reported by the same author. In agreement with the present findings, Havlicek (1967), saw depression of gross behavior of rats following DL-threo-DOPS administration. These inconsistencies could be due to species variation between rats and mice. However, this is unlikely since Creveling et al. (1968) found that DL-threo-DOPS did not reverse reserpine-induced central depression in mice.

In the present studies the degree of central norepinephrine alterations was evaluated by histochemical and biochemical analysis. From Tables 3 and 4, the norepinephrine values (expressed as a percentage of the hypothalamic value) were: midbrain (43\%), hippocampus (34\%), brain stem $(33 \%)$, striatum (29\%) and cortex (16\%). Thus, the largest concentration of norepinephrine was found in the hypothalamus, while the cortex exhibited the lowest amount of the transmitter. These results differ somewhat from those reported by other investigators. Maickel et al. (1968), who assayed discrete areas of rat brain (using a modified fluorimetric procedure, and a different manner of brain dissection from the present method), found the norepinephrine contents in the medulla and midbrain to be $59 \%$ and $66 \%$ of the hypothalamic value, respectively. Saelens et al. (1967) reported norepinephrine values to be $56 \%$ in the medulla and $47 \%$ in the midbrain, compared to the hypothalamic concentration. These investigators developed the assay procedure employed in this laboratory. Another study (Glowinski and Iversen, 1966) reported the following values (expressed as percentage of hypothalamic valuel: medulla oblongata $(40 \%)$, midbrain (20\%), 
striatum (14\%), cortex (13\%) and hippocampus (11\%). A1though these studies do not agree with respect to the relative amounts of norepinephrine in various brain regions, they all demonstrate highest amount of the transmitter in the hypothalamus. A possible source for the different values reported may be the different experimental methods used. For example, in the present studies, protein in the brain tissue was precipitated in the presence of hydrochloric acid instead of perchloric acid (employed in the original assay by Saelens et al., 1967). Further, the homogenate was prepared from crushed tissue instead of homogenized tissue. In addition, brain dissection and separation of brain areas followed anatomical descriptions according to the rat atlas of Konig and Klippel (1963). All nerves on the ventral brain surface, the geniculate bodies and the cerebellum were removed and discarded.

A second source of error is the length of time taken for sample preparations. As indicated by Gutman and Weil-Malherbe (1967), central norepinephrine values can differ by as much as $32 \%$, if the time interval between death of the rat and freezing of its brain is shortened from 79 to $21 \mathrm{sec}$. Saelens et al. (1967) did not weigh their samples. This shortened their time of sample preparation and could account for some of the variation seen in their values compared to those obtained in this study. However, since norepinephrine values were expressed in terms of $\mathrm{ng} . / \mathrm{gm}$. protein in the Saelens study, it is difficult to compare and relate their results with the present findings. Gutman and Neil-Malherbe (1967) also found that immediate 
plunging of dead rats into 7 iquid nitrogen resulted in faster cooling of the brain. This procedure produced norepinephrine concentrations twice as high as those samples obtained from brains, dissected at $4^{\circ} \mathrm{C}$ without immediate freezing. Therefore, norepinephrine concentration in brain areas can decrease as a function of the time taken for preparing the tissue sample.

In addition, a circadian rhythm for norepinephrine concentration exists in the rat. Friedman and Walker (1968), showed that norepinephrine levels in the caudate nucleus and the midbrain reached a peak at 6:00 a.m., but had their lowest values at $6,00 \mathrm{p.m}$. In the cat, Reis et al., 1968, measured norepinephrine regional1y, 4 times in a $24 \mathrm{hr}$. period and found the cervical cord, anterior hypothalamus, the tuber cinereum and the pineal gland to have a circadian rhythm. The regional rhythms were not synchronized. For example, the pons, the substantia nigra and the lateral tegmentum had peak norepinephrine values at 1:00 p.m. while the cervical cord, the region of the tuber cinereum and the anterior hypothalamus exhibited their highest values at 7:00 p.m. Further, the pineal gland had peak norepinephrine concentration at 7:00 a.m. Some regions of the brain (e.g., the superior colliculus and the lateral thalamus) had biphasic daily rhythms and were ultradian. In addition, these workers found that norepinephrine concentrations fluctuated smoothly over the day in certain areas (e.g., anterior hypothalamus and the pineal gland), whereas others showed a steep rise only at one sampling time (e.g., cervical cord and the pons). Circadian rhythm for norepinephrine in the rat has also been reported 
by other workers (Manshardt and Wurtman, 1968; Scheving et al., 1968). Since the rat studies were not as detailed as the Reis study, it is difficult to say whether multiple or individual rhythms for norepinephrine exist regionally in the rat brain. However, as norepinephrine values are not stable over $24 \mathrm{hr}$., the time of decapitation of the rat becomes very important and can contribute to the difference in norepinephrine concentrations obtained in this study compared to that reported by other investigators (Glowinski and Iversen, 1966; Saelens et al., 1967; Maickel et al., 1968). In the present study, all rats were decapitated between 6:00 p.m. and 8,30 p.m., a time period during which norepinephrine levels in the brain have been reported to be low (Freidman and Walker, 1968). Unfortunately, most of the other investigators did not indicate the time of sacrifice of their animals. This could account for the lower norepinephrine values found in the various brain regions in this study as compared to those reported by Maickel et al. (1968). For example, in the midbrain, the control group showed a norepinephrine content of $168 \mathrm{ng} . / \mathrm{gm}$. (Table 3), while a value of $650 \mathrm{ng} . / \mathrm{gm}$. was seen in the Maickel study (1968), demonstrating a 4 fold difference. Friedman and Walker (1968) saw a 2 fold difference in norepinephrine concentration in the midbrain of the rat due to circadian rhythm, whille a 4 fold difference occurred in norepinephrine concentration in the cat mesencephaton due to diurnal variation (Reis et al., 1968).

When compared to spectrometric assays, this present radiometric procedure which employs phenylethanotamine transferase and S-adeno- 
sylmethionine-methy $1-14 C$, is much more specific for norepinephrine. Physiological concentrations of dopamine, metanephrine, normetanephrine and epinephrine do not interfere, since they have Michaelis-Menten constants $(\mathrm{Km})$ from 400 to 10,000 times larger than that for norepinephrine (Saelens et al., 1967). This specificity of the assay for norepinephrine may also contribute to the lower values obtained in this study compared to previous reports, where extensive sample manipulations can introduce high fluorescent values (Glowinski and Iversen, 1966; Friedman and Walker, 1968; Maickel et al., 1968).

The biochemical studies show that DL-threo-DOPS produced variations in repletion of norepinephrine in the different brain regions studied (see Tables 3 and 4). As indicated in the introductory part of this section, DL-threo-DOPS is decarboxylated in vivo to 1-norepinephrine. The biochemical data suggest that the synthes is of 1 -norepinephrine from DL-threo-DOPS probably differs in various brain regions - higher in the hippocampus, cortex and the corpus striatum, but lower in the brain stem, midbrain and the hypothalamus. Another factor could be variation in the transport of the amino acid precursor to various parts of the brain. Support for these suggestions comes from the observations of Glowinski and Iversen (1966). These workers saw higher levels of H3-dopamine and H3-norepinephrine in the medulta oblongata and the striatum compared to the hypothalamus, the hippocampus and the cortex, $2 \mathrm{hr}$. after intraventricular injections of H3dopa. Five hr. after injection, highest concentrations of H3-dopamine and H3-norepinephrine occurred in the hypothalamus and the lowest con- 
centrations in the cortex. Since dopa is also a substrate for dopa decarboxylase which converts it to dopamine (Holtz, 1959), these results indicate a variation in the rate of dopamine formation in different brain areas after dopa administration and further suggest that dopa decarboxylase activity in different brain areas is not uniform. In a later investigation, Iversen and Glowinski (1966) studied the turnoyer of norepinephrine in the cerebellum, hippocampus, cortex, hypothalamus and the medulla oblongata by 3 different methods. They observed that the rates of norepinephrine turnover were lower in the hypothalamus and the medulla oblongata but were higher in the cortex and the hippocampus, with the cerebellum having a turnover rate about twice that of the hypothalamus. These workers noticed that brain regions with high concentrations of endogenous norepinephrine and those which accumulated greater amounts of exogenous H3-norepinephrine when introduced intraventricularly, tended to have slower rates of turnover. The present study yielded data which agree with these observations, since the brain stem (pons-medulla oblongata), hypothalamus and the midbrain had lower elevations of norepinephrine concentration, compared to the hippocampus, cortex and the corpus striatum in the rats receiving DL-threo-DOPS per se.

These results disagree with the observation of Carlsson (1964), who saw little accumulation of norepinephrine in the whole brains of mice treated with DL-threo-DQPS. Perhaps, estimation of the regional norepinephrine concentrations by the present method revealed subtle increases which are masked when the whole brain is taken for analysis. 
In support of this statement is the observation that differences in norepinephrine concentration between drug and control groups were only significant $(P<0.05)$ in the brain stem, the hippocampus and the cortex.

From seizure threshold changes in rats following treatment with reserpine, $\alpha-m T, D L-t h r e 0-D O P S$ and combinations of these compounds, support for the hypothesis that lack of norepinephrine enhances seizure susceptibility has been provided.

As discussed previousiy, the actions of catecholamines at effector sites have been classified into alpha and beta effects. At present, compounds which selectively block either type of receptors are available. Thus, alpha and beta adrenergic blocking agents have been employed by investigators for the characterization of adrenergic effects. In the present study, representative alpha and beta adrenolytic agents were examined for their actions on susceptibility of rats to seizures induced via hippocampal stimulation. The alpha adrenergic blocking compounds employed were phenoxybenzamine and phentolamine.

Phenoxybenzamine is a haloalkyamine. It cyclizes to a highly reactive and unstable ethyleneimmonium ion in vivo. This reacts readily with nucleophilic groups (Harvey and Nickerson, 1953; Nickerson, 1965; 1967). Blockade of alpha adrenergic action is probably achieved by the formation of a highly reactive carbonium ion when the 3 membered ring breaks (Belleau and Triggle, 1962). Its relatively slow onset is probably due to the formation of these intermediates which act as alkylating agents. The prolonged duration of blockade by phenoxybenzamine 
probably depends on the formation of a covalent bond between the antagonist and the receptor (Yong and Marks, 1969). During the development of blockade by phenoxybenzamine, presence of catecholamines can attenuate and antagonize blockade (Nickerson and Gump, 1949; Furchgott, 1954/, suggesting competition for the same population of receptors. However, once blockade is achieved, it is irreversible (Nickerson, 1965). Phentolamine produces competitive blockade at alpha adrenergic receptors. In contrast to phenoxybenzamine, it is rapid in onset and short in duration (Nickerson, 1965).

In this study, phenoxybenzamine, but not phentolamine reduced thresholds of rats to seizures, induced by hippocampal stimulation (see Figures 6 and 7). A7though both compounds can antagonize alpha adrenergic actions in the periphery, they do not produce exactly the same effects when employed to block central actions. For example, phenoxybenzamine but not phentolamine inhibits the EEG stimulant action induced by i.v. administration of epinephrine, norepinephrine or methoxamine (Goldstein and Munoz, 1961). In addition, phenoxybenzamine per se has central stimulant actions on EEG unlike phentolamine. This is probably due to the ability of phenoxybenzamine, but not phentolamine to deplete catecholamines (Shapiro, 1958; Farrant et al.., 1964; Nyback et al., 1967) through persistent blockade of reuptake of catecholamines (Hertting et al., 1961).

Iontophoretic studies on single neurons in selected brain regions indicate that alpha adrenergic blocking compounds do not all react in the same way. For instance, in olfactory neurons, both dibenamine and phentolamine block the ability of norepinephrine to reduce spontaneous 
discharge rate. Dibenamine's effect is biphasic in some cel1s, where the norepinephrine response is enhanced either prior to onset of norepinephrine inhibition or during the period of recovery from dibenamine electrophoresis (Bloom et al., 1964a; 1964b). Furthermore, in brain stem neurons, where 1-norepinephrine induces both excitation and inhibition, dihydroergotamine and chlorpromazine block the excitatory response, but phentolamine is ineffective (Bradley et al., 1966; Boakes et al., 1968). Additional data indicating the non-uniformity of central effects of alpha adrenergic blocking agents have been supplied by Malcolm et al. (1967). By direct application of drugs onto the cortex, these workers observed that norepinephrine diminished the primary cortical evoked response. Its effect was effectively blocked by phenoxybenzamine, tolazoline and phentolamine. of these 3 alpha antagonists, however, only phenoxybenzamine provided full blockade. Moreover, phenoxybenzamine per se also increased the size of the evoked responses. On prolonged application, phenoxybenzamine and phentolamine depressed cortical responses. These variations between alpha adrenergic blocking agents may be due to the existence of a difference in the affinity or access of these agents to the sites of action of catecholamines.

In the present study, phenoxybenzamine did not alter seizure threshold at doses higher than $0.375 \times 10^{-1} \mathrm{mMoles} / \mathrm{kg}$. (Figure 7). This observation may be a reflection of the total effect of phenoxybenzamine on norepinephrine disposition and metabolism in vivo. Besides its primary blocking action at alpha adrenergic receptors, this compound 
also blocks reuptake of norepinephrine into axonal terminals following electrical stimulation. Support for this concept is derived from studies which demonstrated that phenoxybenzamine like cocaine, imipramine and desipramine, blocks the uptake of circulating norepinephrine by nerve endings (Hertting et al., 1961; Axelrod et al., 1962). Furthermore, Euler and Lishajko (1968) observed that phenoxybenzamine antagonized reuptake of $\mathrm{H3}$-norepinephrine in isolated adrenergic nerve granules. Such a mechanism could increase the level of transmitter at effector sites. If norepinephrine inhibits the precipitation of seizures, then depression of seizure susceptibility could ensue via this mechanism. This would oppose the seizure threshold depression that could result from inhibition at al pha adrenergic receptors. Interaction of these two actions could account for the observed normal seizure threshold following high doses of the drug.

Increases in norepinephrine concentration can also result from enhanced synthesis of the compound via stimulation of tyrosine hydroxylase. Evidence that phenoxybenzamine augments catecholamine synthesis is provided by Diarman et al. (1968). They showed that in phenoxybenzamine treated animals, the formation of radioactive norepinephrine and epinephrine in various tissues were elevated when 1-tyrosine-14C was administered, but not when 1-dopa-3H was used as the precursor. This indicates stimulation of tyrosine hydroxylase activity.

Increased norepinephrine levels also occur when extraneuronal accumulation and metabolism of norepinephrine are inhibited. As illustrated by Iversen $(1964,1965)$, phenoxybenzamine has this effect. These 
workers observed that in sympathetic neurons treated with cocaine, phenoxybenzamine further reduced the accumulation of norepinephrine and its metabolites. Additional evidence is provided by Eisenfeld et al (1967), who reported further decreases in the concentration of H3-norepinephrine and its metabolites in cocaine treated or immunosympathectomized preparations. Since immunosympathectomy removes sympathetic neurons and cocaine treatment blocks neuronal uptake of biogenic amines, the above reports suggest an inhibition of extraneuronal accumulation of norepinephrine by phenoxybenzamine. In accordance with the hypothesis that norepinephrine inhibits the genesis of seizures, an increase of adrenergic transmitter (via inhibitions of neuronal uptake and extraneuronal accumulation) at alpha adrenergic receptors would reduce seizure responsiveness. This could antagonize the ability of phenoxybenzamine to increase seizure susceptibility via an alpha adrenolytic effect or through the inhibition of norepinephrine synthesis. The interactions of these two opposing effects on seizure excitability may account for the failure of the drug at high doses to alter seizure threshold.

Like phenoxybenzamine, phentolamine blocks spontaneous release of norepinephrine from isolated adrenergic nerve granules as well as the reuptake of H3-norepinephrine (Bygdeman, 1968; Euler and Lishajko, 1968). Extraneuronal accumulation of norepinephrine in heart (cocaine treated or immunosympathetomized) is also inhibited by phentolamine (Eisenfeld, 1967). Phentolamine can increase norepinephrine synthes is from 1-tyrosine-14C, but not from 1-dopa-3H (Diarman et a1., 1968), indicating an ability to enhance tyrosine hydroxylase activity. 
The above studies indicate that phenoxybenzamine and phentolamine can change the adrenergic tone via a number of mechanisms besides blockade at alpha adrenergic sites. When these compounds elicit a behavioral change, such an effect results from all these factors interacting together.

Additional studies were also conducted with phenoxybenzamine and phentolamine in animals treated with reserpine, $\alpha-m T$ and DL-threoDOPS. Experimental conditions were similar to those previously described. The results are presented in Figure 12. Neither phenoxybenzamine $\left(0.375 \times 10^{-1}\right.$ mMoles $/ \mathrm{kg}$.) nor phentolamine $\left(0.50 \times 10^{-1}\right.$ mMoles $/$ kg.) reduced seizure thresholds any further in such animals. The mean seizure thresholds ( 75 to $80 \%$ of the normal value) were similar to the mean seizure threshold in animals receiving reserpine, $\alpha-m T$ and DL-threo-DOPS. However, in the presence of the alpha adrenergic antagonists, all of the animals had lowered seizure thresholds, whereas in the catecholamine-deficient animals receiving only DL-threo-DOPS there was a tendency for this group of rats to exhibit normal seizure thresholds. Although a definite antagonism of DL-threo-DOPS action on seizure threshold by phenoxybenzamine and phentolamine is not established, these data are still consistent with the hypothesis that lack of norepinephrine enhances seizure susceptibility. Since phenoxybenzamine and phentolamine can block uptake of neurotransmitters at axonal terminals, it is also likely that such compounds may inhibit the uptake of DL-threo-DOPS into neurons, where decarboxylation into 1-norepinephrine probably occurs. The end result of such an action 
Figure 12. Effect of phenoxybenzamine and phentolamine on seizure thresholds in animals with altered catecholamine levels. Threshold Ratio was determined by dividing the drug seizure threshold or the control seizure threshold by the stable seizure threshold. The vertical bars denote $95 \%$ confidence limits. $\Delta$, reserpine $+\alpha-\mathrm{mT} ; \mathbf{0}$, reserpine $+\alpha-m T+$ DL-threo-DOPS; $\diamond$, reserpine $+\alpha-m T+$ DL-threo-DOPS + phenoxybenzamine; $>$, reserpine + $\alpha-m T+D L-$ threo-DOPS + phentolamine, $O$, control. 
THRESHOLD RATIO

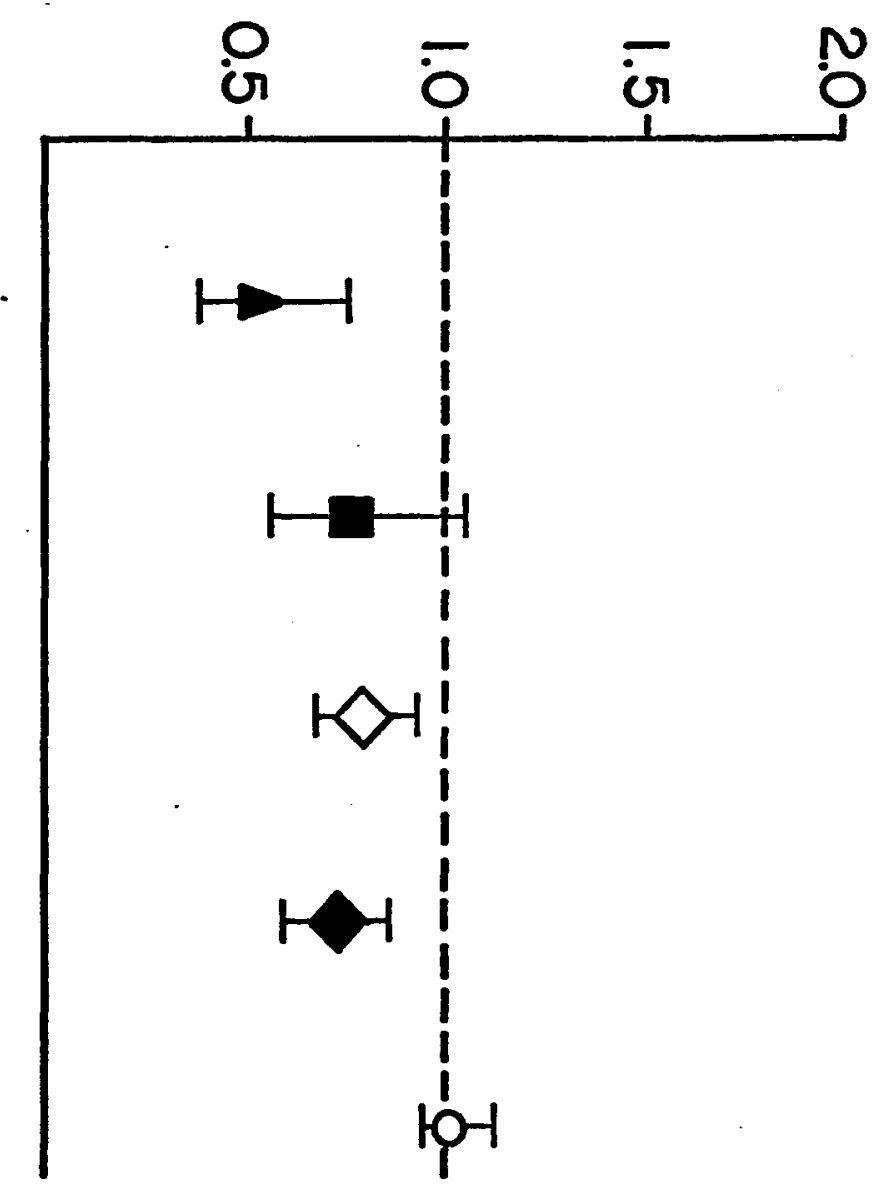


would be a reduction of 1-norepinephrine formation; hence less repletion after treatment with these adrenolytic compounds.

In the presence of phenoxybenzamine, rats treated with reserpine, $\alpha-m T$ and DL-threo-DOPS were sedated with little exploratory behavior. However, only minimal seizures were exhibited at threshold current by these animals. Since maximal seizures and subsequent death occurred in rats receiving only the depleting agents and the amino acid precursor, these results suggest that phenoxybenzamine attenutates seizure severity. This unexpected finding conflicts with the hypothesis that norepinephrine depresses seizure susceptibility. On the other hand, phentolamine treatment provided no indication of alleviation of seizure severity in animals treated with reserpine, $\alpha-m T$ and DLthreo-DOPS. Half of these animals exhibited maximal seizures and 4 out of 7 animals died after convulsive episodes. Since this agent also blocks at alpha adrenergic receptors, the observation that phenoxybenzamine attenuated seizure severity is probably not due to inhibition at these sites.

These data show that phenoxybenzamine and phentolamine do not exert identical influence on the susceptibility of rats to seizures induced by hippocampal stimulation.

The effects of phenoxybenzamine and phentolamine on seizure excitability have been studied to a limited degree by other investigators. As shown in the Introduction, both compounds can antagonize the anticonvulsant effect of acetazolamide and lower thresholds in animals to electrically-induced extensor seizures. Further, phenoxybenzamine 
reduces the seizure threshold of mice to low frequency electroshock seizures. These findings indicate that alpha adrenergic blockade probably enhances the seizure susceptibility of animals.

Although all the data obtained in the present study are not conclusive with regard to the involvement of alpha adrenergic receptors in seizure expression, most are consistent with the findings by other investigators, and indicate that blockade at alpha adrenergic receptors probably enhances seizure susceptibility of animals.

The beta adrenergic blocking compounds, propranolol, pronetha101, sotalol and the 2 isomers of INPEA appear to have different influences on the susceptibility of rats to seizures induced by hippocampal stimulation (Figures 8 to 11). Propranolol produced marked el evation of seizure threshold and protected some animals against convulsions even when the highest current was administered (Figure 8 ). These observations suggest an anticonvulsant action by the drug, and agree with the findings of other investigators. As indicated in the Introduction, propranolol antagonizes tonic leptazol seizures, tonic electroshock seizures, nicotine and strychnine-induced convulsions (Murmann et al., 1966). In addition, it raises thresholds in mice to low frequency electroshock seizures (Yeoh and Wolf, 1968). Such an action may be related to the potent beta adrenolytic property of propranolol (Black et al., 1964; Foo et al., 1968; Hahn, 1968; Raper and Wale, 1968). However, propranolol also has other actions. For example, it possesses reversible alpha adrenergic blocking effect in rabbit aortic strips (Kohli and Ling, 1967; Gulati et al., 1969). Propranolol can 
also inhibit reuptake of norepinephrine at neuronal sites, as demonstrated by inhibition of H3-norepinephrine reuptake into isolated adrenergic nerve granuels (Euler and Lishajko, 1968) and into human blood platelets (Bygdeman, 1968). Since these actions modify the degree of noradrenergic stimulation of receptor sites, they could contribute to the final seizure excitability of the test animal. In addition, propranolol has significant local anesthetic action which is 2 to 3 times greater than procaine (Morales-Aguilers and VaughanWilliams, 1965; Barrett et al., 1968). Such an action could depress synaptic transmission (Taverner, 1960) and could be responsible for the anticonvulsant effect seen with propranolol. At toxic doses propranolol depresses the central nervous system (Black et al., 1964), suggesting that non-specific depression could also account for its anticonvulsant effect. This, however, is unlikely as demonstrated by the current observation that pronetha101, which has central excitatory effects (B1ack and Stephenson, 1962) also elevated seizure threshold (Figure 9). With pronetha101, however, no observable alteration in seizure susceptibility followed high doses of the compound. This suggests complications from nonspecific central excitation at high doses. In fact, at toxic doses, the compound per se produces convulsions (Black and Stephensen, 1962; Morales-Aguilers and Vaughan-Williams, 1965).

Other workers employing pronethalol also reported its ability to reduce susceptibility of animals to maximal electroshock, low frequency electroshock and metrazol-induced seizures. Pronethalol also potentiates the anticonvulsant action of metazolamide and acetazolamide (see Introduction). 
Like propranolo1, pronethalol also exhibits alpha adrenolytic activity as illustrated by its antagonism of norepinephrine-induced contraction of the isolated vas deferens (Ariens, 1967) and in the rabbit aortic strip (Gulati et al., 1969). Pronethalol also inhibits the reuptake of H3-norepinephrine in isolated adrenergic nerve granules (Euler and Lishajko, 1968) and in human blood platelets (Bygdeman, 1968). In addition, pronethalol blocks the extraneuronal accumulation of H3-norepinephrine in cocaine and immunosympathectomized preparations (Eisenfeld et al., 1967) and decreases endogenous norepinephrine levels following chronic treatment (Westfall, 1967). Thus, the ability of pronethalol to modify noradrenergic accumulation in vivo could influence its effect on seizure excitability. An additional factor that may account for the anticonvulsant action of pronethalol is its local anesthetic action (Somani and Lum, 1965). In this respect, pronetha101 resembles propranolol and is about twice as potent as procaine.

Another beta adrenergic blocking agent used in this study; i.e., sotalo1, showed no influence on threshold to seizures induced by electrical stimulation of the hippocampus (Figure 10). This is in general agreement with the results obtained by Lish et al. (1965) who reported no change in seizure susceptibility following the drug in animals subjected to other types of electrically-induced seizures. Moreover, sota101 has been shown to oppose the anticonvulsant action of metazolamide and to reduce seizure thresholds of mice to electrically-induced extensor seizures of the hind limbs (see Introduction). Thus, not all beta adrenergic blocking compounds depress seizure excitability. With 
high doses of sotalo1, hypoactivity, clonic convulsions, central depression and death are produced (Lish et al., 1965). Unlike propranolol and pronetha101, the compound possesses no local anesthetic action (Lish et al., 1965), suggesting that propranolol and pronethalol probably elevate seizure threshold via this action.

Additional evidence against the involvement of beta adrenergic receptors in seizure excitability is provided from the observations that both the $D(-)$ and the $L(+)$ isomers of INPEA had no effect on seizure threshold in the same study (Figure 11). These data are consistent with those reported by Murmann et al. (1966), who found no al teration of susceptibilities to seizures induced by metrazol, strychnine or tonic electroshock by these compounds. Like pronetha101, INPEA produces central stimulation at high doses, but unlike pronethalol, INPEA lacks local anesthetic action (Almirante and Murmann, 1966).

Even with regard to beta receptor antagonism, the compounds used in the present study differ in potency and specificity of action (Raper and Wale, 1968; Fitzerald, 1969). Although their relative potencies vary with the tissue responses studied, propranolol is generally the most potent antagonist at beta adrenergic receptors.

of the 5 beta adrenergic antagonists investigated, only propranolol and pronethalol elevated thresholds to seizures induced by hippocampal stimulation. These compounds may well have depressed seizure excitability via mechanisms unrelated to beta adrenergic blockade. This view is also held by other workers (Leszkovszky and Tardos, 1965; Murmann et al., 1966). Since both propranolol and pronethalol have potent local anesthetic 
activity unlike sotalol and INPEA, the abilities of propranolol and pronethalol to lower seizure susceptibility may be related to inhibition of axonal conduction by these compounds (Abert and Welin, 1967; Vaughan-Williams, 1967). Support for this concept is provided by the ability of i.v. lignocaine (1\%) to block diisopropyl phosphorofluoridate (DFP)-induced epileptiform activity. Although low doses have no, or slight depressant effects on monosynaptic transmission, larger doses indeed depress synaptic transmission (Taverner, 1960).

In conclusion, the data in this study show decreased seizure thresholds in catecholamine-deficient rats. With repletion of 1-norepinephrine from DL-threo-DOPS metabolism, there seems to be a corresponding decrease in seizure excitability. Phenoxybenzamine lowered seizure threshold in naive animals treated with reserpine, $\alpha-m T$ and DL-threo-DOPS. Even though these data are not conclusive, they support an involvement of norepinephrine in the seizure excitable state. Furthermore, they are consistent with the hypothesis that lack of norepinephrine at al pha adrenergic sites increases the seizure susceptibility of an animal. The experimental findings with the beta adrenergic antagonists suggest that modifications of seizure excitability by some of these compounds occur through an action unrelated to receptor blockade. 


\section{GENERAL DISCUSSION}

A method was designed to produce minimal seizures in rats by electrical stimulation via bipolar, stainless steel electrodes, implanted in the right dorsal hippocampus. The surgery involved was relatively simple; aseptic procedures were not required. In this laboratory, all implanted animals survived the surgical procedure. Except for those which received reserpine and died subsequent to maximal seizures, all other animals lived till the end of the experiment, when they were sacrificed.

Generally, typical minimal seizures (facial clonus, rhythmic jaw movements of fore limb clonus) were exhibited in the implanted rat by the fifth stimulation session. The seizure threshold was high initially, then stabilized and remained relatively predictable over periods of one to two months. Beyond these intervals, there was a tendency for the seizure threshold to fall, indicating that the genesis of seizures became progressively easier with increasing time from implantation and repeated electrical stimulation. Although seizure threshold was quite stable in the same animal within a period of one to two months, it was rather different from animal to animal. This could be due to innate animal to animal variability or to the slight differences in the exact location of the electrode tips in the hippocampus, especially their proximity to fiber tracts. Roelofs (1967) has shown that thresholds 
to seizures are lower if the tips of the electrodes are closer to fiber tracts. As a result of this animal to animal variability, all experiments were conducted employing the same animal as its own control. Additional precautions were taken, whenever possible, using a cross-over design. In the same animal drug and control studies were conducted with a 7 day rest period in between. This proved to be unsuitable in studies employing reserpine, which produces prolonged depletion of endogenous biogenic amines (Anden, 1969). In such tests, the control studies were conducted a day or a week prior to the drug studies.

Since the experimental rats were used repeatedly for drug studies, care was taken to prevent complications from in vivo drug-induced enzyme induction or inhibition (Woodbury, 1969). These factors can change the pattern and the rate of drug metabolism and thus complicate the results. In order to prevent this, all animals were allowed at least 2 weeks' recuperation between drug studies.

The overt seizures produced by the present method are reproducible over a long time (up to a year). Hence, it is suitable for long term investigations of candidate anticonvulsant drugs. Such studies are especially important as the treatment of epileptic seizures involyes chronic therapy. The present procedure is superior to existing methods (e.g., aluminum hydroxide cream, ethyl chloride spray, penicillin, metal plates) for inducing chronic epileptogenic foci because seizures can be induced at the experimenter's convenience. The other methods elicit spontaneous episodes of seizures which are unpredictable. 
This is inconvenient to work with, especially if employed as a standard laboratory screening procedure. Furthermore, the induction of seizures by electrical stimulation is better than that caused by irritation from drugs or metals due to the possible interference from drug-drug interactions. In the present model, the rat is used as the experimental animal. Since it is smaller than the cat, rabbit, dog or baboon, it will, therefore, require relatively less drug. This point becomes important in studies employing multiple drug schedules of potential anticonvulsant agents.

Compared to other electrical methods, the present procedure has the advantage of using a freely moving experimental subject. Contact between the experimenter and the rat is minimal. In addition, stimulation is localized to a specific brain area. This is more representative of true epileptic seizures compared to seizures induced via corneal or ear electrodes, where massive electrical stimulations are more likely to cause stress and therefore influence the hormonal status in the experimental animal. Moreover, the method is flexible. By altering the current intensity, both minimal and maximal seizures may be produced. Finally, the actual current which produces a seizure is measured in this procedure. This is better than methods which measure only the incidence of seizures, since the latter procedures measure an all or none response and not a graded response. Measurement of a graded response is always more desirable since seizure susceptibility varies from animal to animal in the same population. 
The gross behavior and general condition of the electrode implanted rat (evaluated as the amount of weight gain and exploratory behavior) were essentially normal. However, in the older animals, tissue rejection of the dental acrylic was observed. There was a tendency for a dried secretion to form between the acrylic and the skull. Over time this built up and eventually pushed the dental acrylic and the electrodes away from the sku11, thus rejecting them. By scraping away the dried secretion periodically, this problem was minimized. Another interesting observation was a tendency for the skull, especially in the region above the cerebellum to become thickened with time of implantation. This produced suppression and deformity of the cerebellum. Since this structure controls motor co-ordination, the presence of skull thickening can influence behavior. Such animals were, therefore, not employed further in the study and were immediately sacrificed.

The minimal seizures produced by stimulation of the hippocampus resemble minimal seizures induced by other experimental procedures in their response to diphenylhydantoin and trimethadione. Since low doses of trimethadione, but not diphenylhydantoin raised seizure threshold, it is possible that these seizures are elicited and maintained by a mechanism which involves electrical discharges that form a recurrent self-oscillating cortico-thalamic loop; a mechanism independent of post-tetanic potentiation. At a high dose, the nonspecific membrane 
stabilizing influence of diphenylhydantoin is probably responsible for the slight seizure threshold elevation observed following this drug. The results obtained with alteration of catecholamine levels in vivo by means of reserpine, $\alpha-m T$ and DL-threo-DOPS indicate that seizure susceptibility did indeed change following treatment with these drugs per se or in combination. The rats were more responsive to seizures after depletion of catecholamines, while repletion of 1norepinephrine with DL-threo-DOPS antagonized this effect. These findings generally support the hypothesis that norepinephrine acts as an inhibitory transmitter in the manifestation of seizures.

Although not conclusive, the results obtained with the alpha adrenergic blocking agents, phenoxybenzamine and phentolamine, indicate that these compounds enhanced seizure susceptibility. Since inhibition at alpha adrenergic receptors would reduce the action of norepinephrine, these results are consistent with the stated hypothesis.

As discussed earlier, there are many studies which implicate the influence of catecholamines on seizure expression (see Chapter IV). Most of these findings support the above hypothesis. The general observation is that elevation of norepinephrine level reduces seizure susceptibility, while depletion of norepinephrine enhances seizure excitability. Such studies have employed precursors of norepinephrine, norepinephrine per se, metabolic inhibitors, e.g., monoamine oxidase inhibitors; catechol-0-methyl transferase"poisons,"as well as agents which interfere with the synthesis (e.g., $\alpha-m T, \alpha-m m T, \alpha$-methyl$D O P A$, disulfiram), the uptake (imipramine, desipramine) and the storage 
of norepinephrine (e.g., reserpine, tetrabenazine). A more recent study (Browning and Maynert, 1970) employed intracisternal injections of 6-hydroxydopamine for inducing chronic depletion of norepinephrine and dopamine. In such rats, susceptibility and severity to seizures were enhanced. This was represented as depression of threshold to facial clonus, prolongation of the tonic phase and post-ictal depression of maximal electroshock and lengthening of seizures as well as postictal depression following fluorothyl-induced seizures.

When considered together, these observations provide strong support for the hypothesis that norepinephrine has a role in preventing the precipitation of seizures. However, since the central excitable state of an animal results from the activities of neurohormones interacting together, it is impossible to exclude the influence of other neurotransmitters in the control of seizure susceptibility. This concept is further emphasized by a recent report (Hanigan et al., 1970) which indicates a distinct inverse relationship between the adrenergic and serotoninergic systems relative to seizure latency and duration. Enhancement of the adrenergic component and/or inhibition of the serotoninergic component increased seizure latency and reduced seizure duration. In addition, al though the cholinergic system also affects seizure latency, it could not be related to either the adrenergic or serotoninergic systems.

Additional support for the working hypothesis that norepinephrine acts as an inhibitory transmitter in the genesis of seizures can be obtained by closely examining the influence of combined treatment with 
DL-threo-DOPS and monoamine oxidase inhibitors on seizure threshold employing the present model. Such experiments will provide considerable information to confirm the above hypothesis.

The final part of the study which detailed the effects of several compounds with adrenergic beta blocking action on seizure susceptibility show that propranolol and pronethalol had anticonvulsant activities, but that sota101, $D(-)$ and $L(+)$ INPEA did not. These agents demonstrate varying potencies in their ability to inhibit beta adrenergic effects (see Chapter IV). Since all these compounds do not influence seizure susceptibility in the same way, the abilities of propranolol and pronethalol to alleviate hippocampal-induced seizures are probably unrelated to their effects at beta adrenergic receptors. Since these compounds have actions on cholinergic as well as histaminergic sites and some possess considerable local anesthetic property, their influence on seizure excitability could be related to these effects (Raper and Wale, 1968). These findings agree with the conclusion of Leszkovszky and Tardos (1965) and Murmann et al. (1966) that certain beta adrenergic blocking agents alter seizure susceptibility by mechanisms unrelated to their actions on beta adrenergic receptors.

In conclusion, this study presented data which support the hypothesis that norepinephrine acts as an inhibitory transmitter in the genes is of minimal seizures induced by hippocampal stimulation. There was some indication that activity at alpha adrenergic receptors might be implicated, whereas beta adrenergic receptors do not seem to be involved. 


\section{SUMMARY AND CONCLUSIONS}

A study was undertaken to design a new method for producing minimal seizures in rats implanted with bipolar, stainless steel electrodes in the right, dorsal hippocampus. These seizures were elicited by repetitive electrical stimulation and were reproducible over time. Furthermore, individual rats exhibited relatively stable seizure thresholds.

Trimethadione readily reduced the seizure susceptibility in these animals and protected several rats from seizures. Diphenylhydantoin had no effect on seizure threshold in these rats except at a high dose. It was suggested that these seizures resembled other types of minimal seizures produced experimentally.

Susceptibility of rats to such seizures was increased after treatment with reserpine and $\alpha-\mathrm{mT}$ per se or in combination. When animals depleted of catecholamines were injected with DL-threo-DOPS, the 1-norepinephrine precursor, seizure susceptibilities tended to revert towards normal. In addition, alpha adrenolytic agents like phenoxybenzamine or phentolamine enhanced seizure excitability when administered to naive rats or animals with altered catecholamine levels (e.g.,treated with reserpine, $\alpha-m T$ and DL-threo-DOPS). These results provided support for the hypothesis that norepinephrine is 
an inhibitory transmitter in seizure expression and implicated the involvement of alpha adrenergic receptors in these phenomena..

However, examination with a series of beta adrenergic blocking compounds showed that changes in seizure susceptibility by certain of these agents were probably not related to their ability to block beta adrenergic receptors. 


\section{APPENDIX}

\section{A. Electrode Implantation}

i. Apparatus - air jet, atlas of the rat brain (Konig and $\mathrm{KTi}$ ppel, 1963), cotton pellets (Nos. 1 and 4; Richmond Dental Cotton Co.), dental acryl ic (Nuwe1d, L.D. Caulk Co., Milford, Del.), dental drill (Fordom Electric Co. Inc., Bethel, Conn.), dental bits (No. 57, straight hand piece; The Ranson and Randolph Co., Toledo, Ohio), electrodes (bipolar, stainless steel, $0.3 \mathrm{~mm}$. diameter, MS'4303-312"SS-010"; Plastic Products Co., Roanoke, Virginia), hair clippers (Model JD; Andis Clipper Co., Racine, Wis.), machine screws (0.80, 1/8", round head, 18-8 S.S.; Harper, 111.), stereotaxic (Baltimore Inst. Co.), surgical instruments, suture needles (Ethicon Inc., Sommerville, N.J.), suture thread (silk, size 0, black braided U.S.P.; Ethicon, Inc., Sommerville, N. J.)..

ii. Chemicals - epinephrine (1:1000 Adrenalin, Parke Davis \& Co., Detroit, Mich.), pentobarbital (Dibutal, Diamond Lab. Inc., Des Moines, Iowa), saline, vaseline, wax, xylocaine (2\% Xylocaine Hydrochloride; ASTRA Pharmaceutical Products, Inc., Worcester 6, Mass.).

iii. Procedure - The bipolar electrodes were fixed onto the stereotaxic apparatus and the anterior-posterior position at ear bar zero (EBO) was recorded. The rat was anesthetized with pentobarbital (45 $\mathrm{mg}$. /kg.); the dorsal part of its head was shaved and it was placed in $: .$. 
the stereotaxic apparatus with its head centered, the angle of the jaw bar being positioned according to the atlas of Konig and Klippel (1963). For most animals, the bar was placed at $5 \mathrm{~mm}$. A central incision extending from the level of the eye to the neck region (about $30 \mathrm{~mm}$.) was then made and the wound was flooded with xylocaine. The skull was exposed, cleaned and bleeding was controlled by cleaning with epinephrine-soaked cotton pellets, bone wax or drying the skull surface with an air jet. The mid-line position of the skull was recorded. This is the saggital zero (SO).

The electrodes were positioned at $2.59 \mathrm{~mm}$. lateral to $\mathrm{SO}$ and 3.80 mm. anterior to $E B O$ on the right side of the rat. This point was marked and a hole was drilled here. Two more holes were made at random distances from the electrode position for setting in 2 machine screws. The electrodes and machine screws were then placed in these holes (the electrodes were lowered $2.50 \mathrm{~mm}$. below the brain surface). Vaseline was applied to the edge of the wound after cleaning and drying it. Acrylic powder was then generously dusted onto the sku11 surface followed by 23 drops of solvent. Excess acrylic was quickly wiped off. (This could become a source of irritation and produce bleeding later). After the acrylic was dry, the wound was infilterated with xylocaine and sutured. The animal was allowed 1 week recuperation from surgery.

\section{B. Verification of Electrode Site and Tissue Damage}

i. Apparatus - dissecting board, embedding blocks (1" $\times 1 " \mathrm{x}$ 3/4"; Scientific Products), ice cube trays (micro size), jars (capped), 
painting brush (smal1), perfusion system ( 2 gàllon jars with outlets connected to a two-way adapter to which was jointed a 20 gauge, 2" injection needle), slides and cover slips, sliding microtome (OmE; Reichert-Wein, Austria), staining dishes (petri dishes), stender dishes, surgical instruments.

ii. Chemicals - celloidin ( $4 \%$ and $8 \%$, i.e., $40 \mathrm{gm}$. parloidin strips in $480 \mathrm{ml} .100 \%$ ethanol and $480 \mathrm{ml}$. anhydrous ether; the parloidin strips were obtained from Mallinckrodt Chem. Works, St. Louis), ethanol $(75 \%$; $95 \% ; 100 \%)$, formal in $(10 \%$ in saline), lithium carbonate (0.5\% in distilled water), pentobarbital (Diabutal, Diamond Lab. Inc.; Des Moines, Iowa), piccoiyte, pyridine, saline ( $0.9 \%$ sodium chloride), solution A $(0.1 \%$ luxol fast blue in $95 \%$ ethanol containing $5 \mathrm{ml} . / 1$. of $10 \%$ acetic acid), solution B ( $0.15 \%$ standard cresyl violet acetate in distilled water), terpineol, xylene.

iii. Procedure - The rat was anesthetized $(45 \mathrm{mg} . / \mathrm{kg} .$, pentobarbital) and perfused with saline, followed by $10 \%$ formalin via the right ventricle. A perfusion pressure of $100 \mathrm{~cm}$. was employed. The brain was removed and stored in 10\% formal in for at least 2 days. It was then washed for $24 \mathrm{hr}$. in running water and then placed in the following solutions in successive order $(24 \mathrm{hr}$. immersion in each solution):

1. Four changes of pure pyridine

2. Four changes of a mixture of half pyridine and half celloidin $4 \%$ 
3. Two changes of $4 \%$ celloidin

4. Two changes of $8 \%$ celloidin

The tissue was then transferred to a stender dish and $8 \%$ celloidin was added to about 3 times the thickness of the tissue. The dish was covered and exposed to air till the celloidin level was reduced to half, at which time the dish was transferred to a dessicator, saturated with chloroform fumes. The hardened tissue block was carved to remove excess celloidin and stored in $70 \%$ ethanol. Storage in ethanol was omitted if the brain was sectioned immediately

For sectioning, the brain was fixed to an embedding block with $8 \%$ celloidin. Frontal sections (30 microns) were cut using a sliding microtome. The tissue specimens were placed in appropriate places in microsize ice cube trays containing $75 \%$ ethanol. They were stained with Solution $\mathrm{A}$ at $57{ }^{\circ} \mathrm{C}$ for $4 \mathrm{hr}$., in a covered dish (precautions were taken to prevent evaporation of the stain). Excess stain was washed with $95 \%$ ethanol, followed with distilled water. The sections were then immersed in $0.5 \%$ lithium carbonate $(15-30 \mathrm{sec}$ ) to begin differentiation. This was followed by washing in 3 changes of $70 \%$ ethanol (at this stage the fibers should be stained blue and the cells should be unstained). The sections were then immersed in distilled water and then stained with Solution B for 3 min. They were then differentiated in several changes of $95 \%$ ethanol, cleared in xylene-terpineol mixture $(1: 1)$ followed with xylene. They were mounted in piccolyte.

Histological examination of each section under light microscope was conducted to verify extent of iesion, site of electrode tract and 
tissue damage. The rat atlas of Konig and Klippel (1963) was used as the reference.

C. Histochemical Assay for Norepinephrine

i. Apparatus- - adhesive tape, alcohol lamp, dessicator, dry box, fluorescence microscope (Carl Zeiss; Germany), glass vials (Vistrex), hot plate oven (Model HPA 1915B, Type 1900; Thermolyne Cor., Dubuque, Iowa), ice cube trays (microsize), lyophilizer (Virtis, Model No.10-145 MR-BA; Gardiner, N.Y.), peel-a-way disposable, plastic tissue embedding molds (size T12; Peel-A-Way, Iowa), polaroid type 107, speed 3000, black and white film (Polaroid Cor., Mass), slides and cover slips, sliding microtome (OmE; Reichert-Wien, Austria), staining dishes (1arge), surgical instruments, tin foil and vacuum oven (National Appliance Co.).

ii. Chemicals - crown immersion oil (Scientific Products), ente11an (Merk; Brinkmann Inst., Inc., Westbury, L.I., N.Y.), freon 12 (dichlorodifluoromethane; Dupont), paraformaldehyde (Fisher), phosphorus penoxide (Baker), notrogen (Liquid), sulfuric acid, wax (Parowax;Sohio).

iii. Procedure - The rat was decapitated and its brain dissected into the forebrain, midbrain, the brain stem and the cerebellum. The specimens were individually placed on tinfoil and quickly "quenched" in freon for 30 seec. Each section was then transferred to a pre-cooled glass vial and capped loosely. It was then immersed in liquid nitrogen to freeze the tissue solid. The cap of each vial was replaced by the metal adaptor of the lyophilizer and attached to the porthole of the 
machine. Freeze drying was conducted out for 5 consecutive days at $-57^{\circ} \mathrm{C}$ and 20-10 $u$ vacuum. The sampleswwere then transferred to a dry box, together with two 16" strips of adhesive tape, a large staining dish with lid containing a prefitted ice cube tray on paraformaldehyde and a dish containing phosphorus pentoxide. The samples were placed in the sealed, dry box for $45 \mathrm{~min}$. after which the specimens were transferred to appropriate spaces on the ice cube tray. The staining dish was sealed with adhesive tape and heated at $80^{\circ} \mathrm{C}$ for 2 hr. (hot prate oven). At the end of the heating period, the dish was removed to a fume chamber and opened to expel the formaldehyde fumes by standing for 1 min.. Individual specimens were then put into tissue molds. Degassed paraffin was added and the molds were heated at $60^{\circ} \mathrm{C}$ for 30 min. in a vacuum oven at 20 " mercury. This produced paraffin blocks with little air bubbles and resulted in homogenous embedding of the tissue. Each mold was solidified by exposure to air and then stored in a dessicator away from light (the specimens would be good for up to 3 months).

Sections (15-20 microns) were cut with a sliding microtome and placed on a glass slide, which was then placed on a hot plate $\left(60^{\circ} \mathrm{C}\right)$ till all the paraffin melted ( 1 min.). The cooled slide may be stored in this condition for 5-10 days. If examined on the same day, the sections were infilterated with entellan and mounted by melting the paraffin gently at $60^{\circ} \mathrm{C}$.

The sections were then viewed under a fluorescence microscope using oil immersion with UV light and two filters (SchottiBG 12 filter 
and a Zeiss 50 filter). Norepinephrine was located as varicosities or fibers with green fluorescence. Photomicrographs of selected sections were taken with type 107 black and white polaroid film (speed 3000) using exposure time of 1-1 1/2 min.

\section{Radiometric Assay for Norepinephrine}

i. Apparatus - centrifuge tubes (ut tracentrifuge), chromatography paper (Whatman No. 1), chromatography tanks (4), gillotine (small animal), glass rod, hammer, ice buckets (3), micropipets ( $10 \mathrm{ul}$. 20 ul.; Clay-Adams; Sargent), Packard Tri-Carb Scintillation Spectrometer and counting vials, parafilm, petri dish, surgical instruments, tin foil, ultracentrifuge, water bath $\left(37^{\circ} \mathrm{C}\right)$.

ii. Chemicals - ammonium hydroxide (Allied Chemicals), butanol (Baker), dry ice, epinephrine, ethanol (100\%), ethylenediaminetetraacetate (Disodium E.D.T.A.; Fisher), hydrochloric acid (Allied Chemi- , ; cals), ice, iodine (Baker), magnesium carbonate (U.S.P. Heavy powder; Mallinckrodt), norepinephrine, pargyline (Abbott), phenylethanolamine$\mathrm{N}$-methyl transferase (PNMT; Seravac), phosphate buffer $(0.1 \mathrm{M}, \mathrm{pH} 7.4)$, dimethyl POPOP (Packard), PPO (Packard), pyrogallol (Baker), S-adenosylmethionine (methyl-C14) ( $52 \mathrm{mCi} / \mathrm{mM}, 10: \mathrm{uCi}$ pack in $0.2 \mathrm{ml}$.; Amersham/Searle Radiochemicals), sulfuric acid (Dupont), toluene (Baker).

iii. Procedure - The rat was decapitated and its brain removed and placed on a coldipetri dish. Ice cold saline was added to moisen the tissue. Using the method of Axelrod (1962), the brain stem, hypothala- 
-mus, midbrain, hippocampus, cortex and corpus striatum were dissected out and immediately frozen in dry ice on tin foil. The frozen tissues were then crushed and immediately transferred to tared, cold centrifuge tubes and weighed. Samples were then homogenized by hand with addition of the respective amounts of homogenizing solution (0.01 M HCl, $1 \mathrm{mM}$ pargyline, $1 \mathrm{mM}$ pyrogal101 and $1 \mathrm{mM}$ E.D.T.A.disodium salt) : brain stem, $0.3 \mathrm{ml}$; hypothalamus, $0.1 \mathrm{ml}$; midbrain, $0.2 \mathrm{ml}$. ; hippocampus, $0.2 \mathrm{ml}$; cortex, $0.6 \mathrm{ml}$; corpus striatum, $0.2 \mathrm{ml}$. About 5 mg. of magnesium carbonate was added to each sample and the homogenate was mixed wel1 and centrifuged at 3000 r.p.m. for 20 min. at $0^{\circ} \mathrm{C}$.

Twenty $\mathrm{ul}$. of the supernatant was transferred to a microcentrifuge tube and to this was added $10 \mathrm{ul}$. PNMT (1 vial containing $14 \mathrm{mg}$. PNMT and $0.5 \mathrm{ml}$. buffer containing 0.1.M phosphate buffer, $\mathrm{pH} 7.4$ and $1 \mathrm{mM}$ E.D.T.A. disodium salt) and $10 \mathrm{ul}$. S-adenosyl-L-methionine-methylCl4 (dilute $0.2 \mathrm{ml}$. vial to $20 \mathrm{ml}$. with $0.01 \mathrm{M}$ sulfuric acid), followed with mixing. The mixture was incubated at $37^{\circ} \mathrm{C}$ for $1 \mathrm{hr}$. ; the reaction: was ended by placing the centrifuge tube in ice.

Twenty 41 . of the incubate was spotted on an $18^{\prime \prime} \times 9^{\prime \prime}$ Whatman No.1 chromatogram paper using 1:1000 epinephrine as the carrier (6-7 samples were spotted on each chromatogram). Separation was achieved by ascending chromatography (12-16 hr.) with a $4: 1$ butanol : hydrochtoric acid (0.1 M) solvent system. The dried chromatogram was exposed to ammonia followed by iodine fumes. Epinephrine spots were located as brown circles with $\mathrm{Rf}$ ofabout 0.17 . These spots were cut out and reacted with $100 \%$ 
ethanol( $4 \mathrm{ml}$.) for $1 \mathrm{hr}$. Fifteen $\mathrm{ml}$. of toluene-based phosphor (T.S.S.) was added and the samples were counted in a Packard Tri-Carb liquid scintillation spectrometer, employing slit width of 50-1000 and a gain of $5.5 /$.

With each batch of experiments (total of 36 samples), a standard norepinephrine curve $(1.25-5.0 \mathrm{ng} . / 20 \mathrm{ul}$.) was carried out, together with a.blank which consisted of the homogenizing solution. Stock solution of standard norepinephrine contained $50 \mathrm{ug} . / \mathrm{ml}$.

iv. Calculations - Disintegrations per min. of each sample was calculated by correcting for quenching and machine efficiency ( the A.E. S. method of counting was employed); the disintegrations per min. (d.p.m.) were multiplied by 2, since only half of the incubation mixture was chromatogramed. A standard curve was constructed and from this the concentration of norepinephrine in $20 \mathrm{ul}$. of the homogenate was deduced. The amount of norepinephrine present in the total homogenate was calculated by assuming brain solids to contain $90 \%$ water by weight (i.e. , total volume $=\mathrm{ml}$. of homogenizing solution added $+90 \%$ brain wt. (gm.). The norepinephrine values were then expressed as $\mathrm{ng} . / \mathrm{gm}$. wet weight of the specific brain tissue. 
Figure 13. A representative norepinephrine standard curve. 


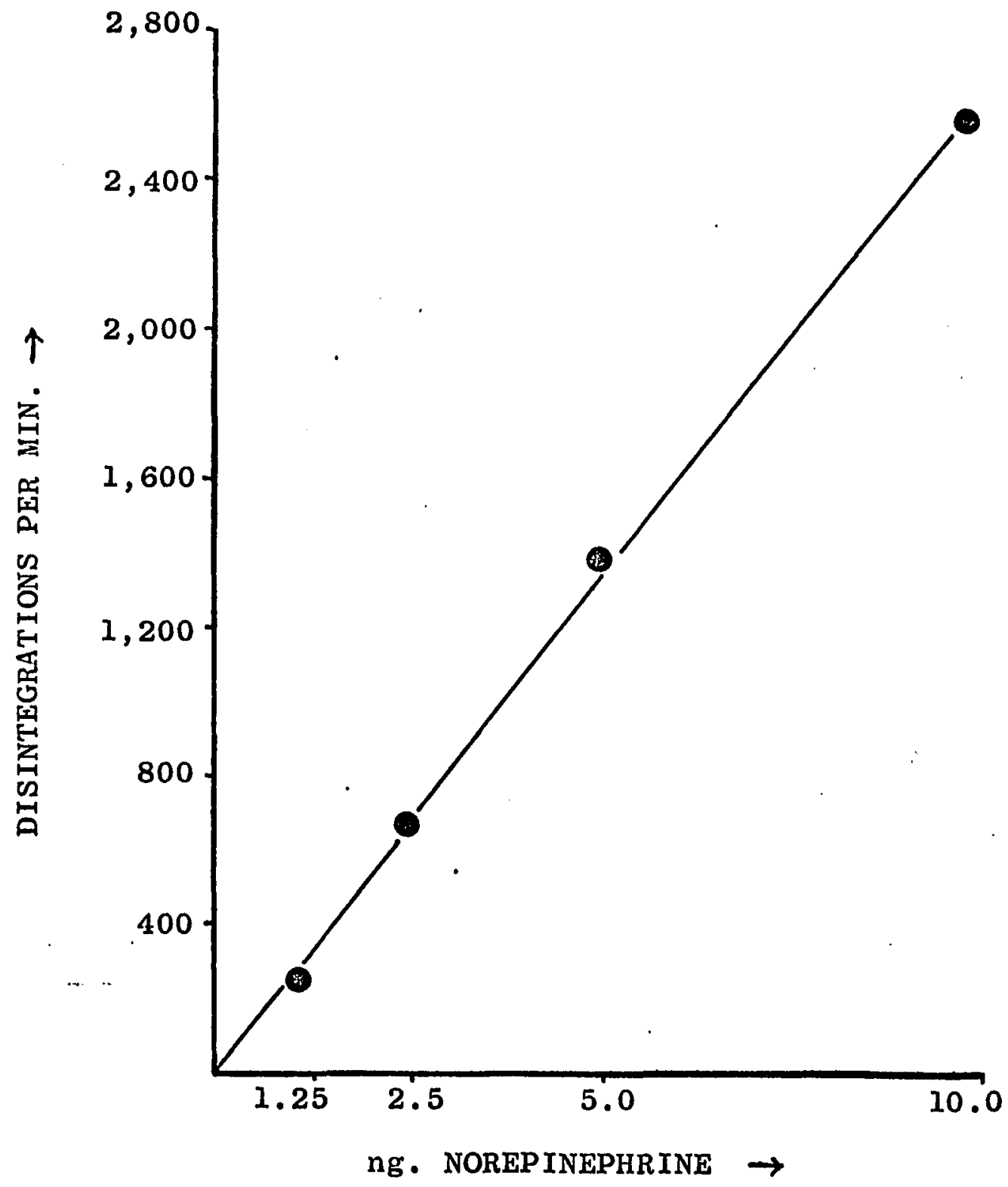




\section{BIBLIOGRAPHY}

Aberg, G. and Welin, I. : Interaction of beta receptor blocking agents with nerve blocking effects of procaine in vitro. Life Sci. $\underline{6}$ : $975,1967$.

Adey, W.R., Kado, R.T., McIlwain, J.T. and Walter, D.0. : Regional cerebral impedance changes in altering, orienting and discriminative responses : the role of neuronal el ements in these phenomena. Exp. Neurol. 15: 490, 1966.

Ahlquist, R.P. : A study of the adrenotropic receptors. Amer. J. Physiol. 153: $586,1948$.

Ahlquist, R.P. : Adrenergic drugs. In: Dri11, V.A. (Ed.) Pharmacology in Medicine. McGraw-Hill Book Co. Inc., New York. 2nd. ed.: 378, 1958.

Albe-Fessard, D., Buser, P. and Minz, B. : Sur une particularite de 1 'activite electrique du cortex chez le lapin thyreoprive chronique. C.R. Acad. Sci. 233: 1059, 1951.

Almirante, L. and Murmann, W. : Relationship between configuration and adrenergic $\beta$-receptor biocking activity of optical isomers of 1-(4-Nitrophenyl-2-isopropylamino ethanot (INPEA). J. Med.Chem. 9: $650,1966$.

Alpers, H.S. and Shore, P.A. : Specific binding of reserpine - association with norepinephrine depletion. Biochem. Pharmacol. 18: :363, 1969.

Anden, N.-E. : Effects of reserpine and a tyrosine hydroxylase inhibitor on the monoamine levels in different regions of the rat central nervous system. Europ. J. Pharmacol. 1: 1, 1967.

Anden, N.-E., Carlsson, A., Dahlstrom, A., Fuxe, K., Hillarp, N.-A. and Larsson, K. : Demonstration and mapping out of nigro-neostriatal dopamine neurons. Life Sci. 3: 523, 1965a.

Anden, N.-E., Carlsson, A. and Haggendal, J. : Adrenergic mechanisms. Ann. Rev. Pharmaco1. 9: 119, 1969. 
Anden, N.-E., Corrodi, H., Dahl strom, A., Fuxe, K. and Hokfelt, T. : Effects of tyrosine hydroxylase inhibition on the amine levels of central monoamine neurons. Life Sci. $\underline{5}: \_561,1966 a$.

Anden, N.-E., Dahlstrom, A., Fuxe, K. and Larsson, K. : Further evidence for the presence of nigro-neostriatal dopamine neurons in the rat. Amer. J. Anat. 116: 329, 1965b.

Anden, N.-E., Fuxe, K. and Hokfelt, H. : The importance of the nervous impulse flow for the depletion of monoamines from central neurons by some drugs. J. Pharm. Pharmacol. 18: 630, 1966b.

Andy, 0.J. and Akert, K. : Electroencephalographic and behavioral changes during seizures induced by stimulation of ammon's formation in the cat and monkey. Electroenceph. Clin. Neurophysiol. 7: Supp1. III, 1953.

Andy, 0.J. and Akert, K. : Seizure patterns induced by electrical stimulation of the hippocampal formation in the cat. J. Neuropath. Exp. Neurol. 14: 198, 1955.

Ariens, E.J. : The structure-actiivty relationships of beta adrenergic drugs and the beta adrenergic blocking drugs. Ann. N.Y. Acad. Sci. 139: 606, 1967.

Axelrod, J., Gorden, E., Hertting, G., Kopin, I.J. and Potter, L.T. : Mechanism of tachyphylaxis to tyramine in the isolated rat heart. Brit. J. Pharmacol. 19: 56, 1962.

Axelrod, J., Inscoe, J.K., Senoh, S. and Witkop, B. : 0-methylation, the principal pathway for the metabolism of epinephrine andmorepinephrine in the rat. Acta. Biochem. Biophysic. (Amst.). 27: 210, 1958.

Axelrod, J. and Laroche, M.J. : Inhibitor of 0-methylation of epinephrine and norepinephrine. Science. 130: 800, 1959.

Axelrod, J. and Tomchick, R. : Increased rate of metabolism of adrenaline and noradrenal ine by sympathomimetic amines. J. Pharmacol. Exp. Ther. 130: 367, 1960 .

Balzer, H., Holtz, P. and Palm, D. : On the mechanism of convulsive effect of hydrazides in mice. Biochem. Pharmaco1. 5: 169, 1960.

Barany, E. H. and Stein-Jensen, E. : The mode of action of anticonvursant drugs on efectrically induced convulsions in the rabbit. Arch. Int. Pharmacodyn. 73: 1, 1946.

Barnes, T.C. : Effect of anticonvulsive drugs on electroencephalographic response to flickering light in unanesthetized rebbits. Fed. Proce. 13: 333, 1954. 
Barrett, A.M., Crowther, A.F., Dunlop, D., Shanks, R.G. and Smith, L.H. : Cardioselective $\beta$-blockade. Arch. Exp. Path. Pharmakol. 259: 152, 1968.

Belleau, B. and Triggle: D.J. : Blockade of adrenergic $\alpha$-receptors by a carbonium ion. J. Med. Pharm. Chem. 5 : 636, 1962.

Bertler, A. : Effect of reserpine on the storage of catecholamines in brain and other tissues. Acta. Physiol. Scand. 51: 75, 1961.

Bertler, A., Hillarp, N.-A. and Rosengren, E. : Some observations on the synthesis and storage of catecholamines in the adrenaline cells of the suprarenal medul1a. Acta. Physiol. Scand. 50: 124, 1960.

Bertler, A. and Rosengren, E. : Occurrence and distribution of dopamine in brain and other tissues. Experientia. 15: 10, 1959.

Besendorf, H. and Pletscher, A. : Influence of isonicotinoyl hydrazides on the central action of reserpine and 5-hydroxytryptamine. Helv. Physio1. Pharmaco1. Acta. 14: 383, 1956.

Bevan, W. : Sound precipitated convursions : 1947 to 1954. Psycho7. Bu17. 52: $473,1955$.

Bielec, S. : Influence of reserpine on the behavior of mice susceptible to audiogenic seizures. Arch. Int. Pharmacodyn. 119: 352, 1959.

Black, J.W., Crowther, A.F., Shanks, R.G., Smith, L.H. and Dornhorst, A. C. : A new adrenergic beta-receptor antagonist. Lancet. 1: 1080 1964.

Black, J.W. and Stephenson, J.S. : Pharmacology of a new adrenergic : beta-receptor blocking compound (Nethalide). Lancet. 2: 311, 1962.

Bloom, F.E., Baumgarten, R.V., 0liver, A.P., Costa, E. and Salmoiraghi, G.C. : Microelectrophoretic studies of adrenergic mechanisms of rabbit olfactory neurons. Life Sci. 3: 131, 1964a.

Bloom, F.E., Costa, E. and Salmoiraghi, G.C. : Analysis of individual rabbit olfactory bulb neuron responses to the microeletrophoresis of acetylcholine, norepinephrine and serotonin synergists and antagonists. J. Pharmacol. Exp. Ther. 146: 16, 1964b.

Bloom, F.E. and Giarman, N.J. : Physiologic and pharmacologic considerations of biogenic amines in the nervous system. Ann. Rev. Pharmacol. 8: 229,1968 . 
Blum, B., Magnes, J., Bental, E. and Liban, E. : Electroencephalographic studies in cats with experimentally induced hippocampal epilepsy. Electroenceph. Clin. Neurophysiol. 13: 340, 1961.

Boakes, R.J., Bradley, P. B., Brookes, N. and Wolstencroft, J,H.: Characteristics of the response of bràin-stem neurons to noradrenaline. Brit. J. Pharmaco1. 32: 417P, 1968.

Bornstein, M.B. : Presence and action of acetylcholine in experimental brain trauma. J. Neurophysio1. 9: 349, 1946.

Bradley, P.B., Wolstencroft, J.H., Hos i , L. and Avanzino, G.L.: : Neuronal basis for the central action of chlorpromazine. Nature. 212: 1425, 1966.

Brodie, B.B., Comer, M.S., Costa, E. and Dlabac, A. : The role of brain serotonin in the mechanism of the central action of reserpine. J. Pharmacol. Exp. Ther, 152: 340, 1966.

Brown, W., Schiffman, D., Swinyard, E.A. and Goodman, L.S. : Comparative assay of antiepileptic drugs by psychomotor seizure test and minimal electroshock threshold test. J. Pharmacol. Exp. Ther. 107: $273,1953$.

Browning, R.A. and Maynert, E.W. : Increased seizure susceptibility in 6-hydroxydopamine-treated rats. Fed. Proc. 29: 966, 1970.

Basquet, H. and Vischniac, C. : Identite d'action du principe sympathomimetique du genet et de 1 'adrenal ine sur la permeabilite capillaire et sur la crise audiogene. C.R. Soc. Biol. (Paris). 144: 398, 1950.

Bygdeman, S. : Effect of $\alpha$ - and $\beta$-receptor blocking agents on platelet aggregation and uptake of noradrenaline. Acta. Physiol. Scand. 73: $28 A, 1968$.

Carlsson, A. : The occurrence, distribution and physiological role of catecholamines in the nervous system. In: Symposium on Catecholamines. Pharmacol. Rev. 11: 490, 1959.

Carlsson, A. : Functional significance of drùg induced changes in brain monoamine levels. Prog. Brain Res. 8: 9, 1964.

Carlsson, A. : Drugs which block the storage of 5-hydroxytryptamine and related amines. In: Erspamer, V. (Ed.) Hanbuch der Exp. Pharmako1. Spring Verlog, Berlin. 529, 1965. 
Carlsson, A., Corrodi, H. and Waldeck, B..: $\alpha$-substituierte dopacetamide als hemmer der catechol-o-methyltransferas unter der enzymatischen hydroxyl ierung aromatischer aminosauren in den catecholamin-metabolismus eingreifender substanzen. Helv. Chim. Acta. 46: 2271, 1963.

Carlsson, A., Dahlstrom, A., Fuxe, K. and Hillarp, N.-A. : Failure of reserpine to deplete noradrenaline neurons of $\alpha$-methylnoradrenaline formed from $\alpha$-methyldopa. Acta. Pharmacol. Toxico1. 22: 270, 1965.

Carlsson, A., Falck, B., Fuxe, K. and Hillarp, N.-A. : Cellulae localisation of monoamines in the spinal cord. Acta Physiol. Scand. 60: 1964.

Carlsson, A., Hillarp, N.-A. and Waldeck, B. : A Mg+t-ATP-dependent storage mechanism in the amine granules of the adrenal medulta. Med. Exp. (Base1). 6: 47, 1962.

Carlsson, A. and Lindqvist, $M$. : In vivo decarboxylation of $\alpha$-methyl Dopa and $\alpha$-methyl metatyrosine. Acta. Physiol. Scand. 54: 87, 1962.

Carlsson, A., Lindqvist, M., Magnusson, T. and Waldeck, B. : On the presence of 3-hydroxytyramine in brain. Science. 127: 471, 1958.

Carlsson, A. and Waldeck, B. : Mechanism of amine transport in the cell membranes of the adrenergic nerves. Acta. Pharmacol. Toxicol. 22: $293,1965$.

Carr, L.A. and Moore, K.E. : Effects of reserpine and $\alpha$-methyl tyrosine on brain catecholamines and the pituitary-adrenal response to stress. Neuroendocrinology. 3 : 285, 1968.

Castellion, A.W., Swinyard, E.A. and Goodman, L.S. : Effect of maturation on the development and reproducibility of audiogenic and electroshock seizures in mice. Exp. Neuro1. 13: 206, 1965.

Chen, G. and Bohner, B. : Antireserpine effects of certain centrally acting agents. J. Pharmacol. Exp. Ther. 131: 179, 1961.

Chen, G., Ensor, C.R. and Bohner, B. : A facilitation action of reserpine on the central nervous system. Proc. Soc. Exp. Biol. Med. 86: $507,1954$.

Chen, G., Ensor, C.R. and Bohner, B. : An investigation on the sympathomimetic properties of phencyclidine by comparison with cocaine and deoxyephedrine. J. Pharmacol. Exp. Ther. 149: 71, 1965. 
Chen, G., Ensor, C.R. and Bohner, B. : Studies of drug effects on electricaliy induced extensor seizures and clinical implications. Arch. Int. Pharmacodyn. 172: 183, 1968a.

Chen, G., Ensor, C.R. and Bohner, B. : Drug effects on the disposition of active biogenic amines in the CNS. Life Sci. 7: 1063, 1968b.

Chow, M.-I. and Hendley, C.D.:: Effect of monoamine oxidase inhibitors on experimental convulsions. Fed. Proc. 18: 376, 1959.

Chusid, J.G. and Kopeloff, L.M. : Use of chronic irritative foci in laboratory evaluation of antiepileptic drugs. Epilepsia. 10: $239,1969$.

Coleman, D.L. : Phenylalanine hydroxylase activity in dilute and non-dilute strains of mice. Arch. Biochem. Biophys. 91: 300, 1960.

Coleman, D.L. and Schlesinger, K. : Effects of pyridoxine deficiency on audiogenic seizure susceptibility in inbred mice. Proc. Soc. Exp. Biol. (N.Y.). 119: 264, 1965.

Cooke, J.P. : Induction by reserpine of irreyersible audiogenic seizure reactions in non-seizure-prone rats. Texas J. Sci. 13: 235,1961 .

Cooke, J.P., Mabe, W.R. and Hopkins, C. : Effect of reserpine upon shock-prone rats under auditory stimulus. Texas 1 . Sci. 12: 443,1960 .

Cooper, A.J., Moir, A.T.B. and Guldberg, H.C. : The effect of electroconvulsive shock on the cerebral metabolism of dopamine and 5-hydroxytryptamine. J. Pharm. Pharmacol. 20: 729, 1968.

Corrodi, H. and Fuxe, K. : The effect of catecholamine precursors and monoamine oxidase inhibition on the amine levels of central catecholamine neurons after reserpine treatment or tyrosine hydroxylase inhibition. Life Sci. 6: 1345, 1967.

Corrodi, H., Fuxe, K. and Hokfelt, T. : Refillment of the catecholamine stores with 3,4-dihydroxyphenylalanine after depletion induced by inhibition of tyrosine-hydroxylase. Life Sci. $\underline{5}$ : $605,1966$.

Corrodi, H. and Hillarp, N.-A. : Fluoreszenmethoden zue histochemischen sichtbarmachung von monoaminen. I. Identifizierung des fluoreszierenden produkte aus model lyersuchen mit 6,7-dimethoxyisochinol in-derivaten und formaldehyd. Helv. Chim. Acta. 46: 2425, 1963. 
Corrodi, H. and Hillarp, N.-A. : Fluoreszenzmethoden zur histochemischen sichtbarmachung von monoaminen. II. Identifizierung des fluoreszierenden produktes aus dopamin and formaldehyd. Helv. Chim. Acta. 47: 911, 1964.

Creveling, C.R., Daly, J., Tokuyama, T. and Witkop, B. : The combined use of $\alpha$-methyltyrosine and threo-dihydroxyphenylserine-selective reduction of dopamine levels in the central nervous system.

Biochem. Pharmaco1: 17: 65, 1968.

Critchley, M. : Musicogenic epilepsy. Brain, 60: 13, 1937.

Crossland, J. : The significance of brain acetylcholine. J. Ment. Sci. 99: 247, 1953.

Crout, J.R., Creveling, C.R. and Udenfriend, S. : Norepinephrine metabolism in rat brain and heart. J. Pharmacol. Exp. Ther. 132: $269,1961$.

Curtis, D.R. and Watkins, J.C. : The pharmacology of amino acids related to gamma-aminobutyric acid. Pharmaco1. Rev. 17: 347, 1965.

Dahlstrom, A. : Observations on the accumulation of noradrenaline in the proximal and distal parts of peripheral adrenergic nerve fibers. J. Anat. (Lond.). 99: 677, 1965.

Dahlstrom, A. and Fuxe, K. : Evidence for the existence of monoaminecontaining neurons in the central nervous system. I. Demonstration of monoamines in the cell bodies of the brain stem neurons. Acta. Physiol. Scand. 62: Suppl. 232, 1964.

Dahlstrom, A. and Fuxe, K. : Evidence for the existence of monoamine neurons in the central nervous system. II. Experimentally induced changes in the intraneuronal amine levels of bulbospinal neuron systems. Acta. Physiol. Scand. 64: Supp1. 247, 1965a.

Dahlstrom, A. and Fuxe, K. : Evidence for the existence of an outflow of noradrenaline nerve fibres in the ventral roots of the rat spinal cord. Experientia. 21: 409, 1965b.

Dahlstrom, A., Fuxe, K. and Hillarp, N.-A. : Site of action of reserpine. Acta. Pharmacol. Toxicol. 22: 277, 1965.

Dahlstrom, A., Fuxe, K., 01 son, L. and Ungerstedt, U. : Ascending systems of catecholamine neurons from the lower brain stem. Acta. Physio1. Scand. 62: 485, 1964. 
Dahlstrom, A. and Haggendal, J. : Studies on the transport and lifespan of amine storage granules in a peripheral adrenergic neuron system. Acta. Physior. Scand. 67: 278, 1966.

DasGupta, S.R., Killam, E.K. and Killam, K.F. : Drug action on rhinencephal ic seizure activity in the cat. J. Pharmacol. Exp. Ther. 122: 16A, 1958.

DaVanzo, J.P., Greig, M.E. and Cronin, M.A. : Anticonvulsant properties of aminooxyacetic acid. Amer. J. Physiol. 201: 833, 1961.

Delgado, J.M.R. : Chronic implantation of intracerebral electrodes in animals. In: Sheer, D.E. (Ed.) Electrical Stimulation of the Brain. U. of Texas Press. 25, 1961.

Delgado, J.M.R., and Mihailloyic, L. : Use of intracerebral electrodes to evaluate drugs which act on the central nervous system. Ann. N.Y. Acad. Sci. 64: 644, 1956.

DeRobertis, E. : Adrenergic endings and vesicles isolated from brain. Pharmacol. Rev. 13: 413, 1966.

DeSalva, S. : Effects of centrally acting drugs in intact and hypophysectomized rats on EST. Arch. Int. Pharmacodyn. 137: 267, 1962.

DeSalva, S. : EST effects on diphenylhydanto in in hypophysectomized rats. Arch. Int. Pharmacodyn. 142: 366, 1963.

DeSchaepdryver, A.F., Piette, Y. and Delaunois, A.L. : Brain amines and electroshock threshold. Arch. Int. Pharmacodyn. 140: 358, 1962.

Diarman, W., Gordon, R., Spector, S., Sjoerdsma, A. and Udenfriend, S. : Increased synthesis of catecholamines in the intact rat following administration of $\alpha$-adrenergic blocking agents. Mol. Pharmacol. 4: $457,1968$.

Dolin, A.0. : Uslovnyi epileptitcheskii pripadok. Arch. Sci. Biol. (Bologna). 4: 1, 1939. Cited by Kreindler, A. : Experimental Epilepsy. Prog. Brain Res. 19: 79, 1965.

Draskoci, M., Feldberg, W. and Haranath, P.S.R.K. : Passage of circulating adrenaline into perfused cerebral ventricles and subarachnoid space. J. Physio1. 150: 34, 1960.

Earle, K.M., Baldwin, M. and Penfield, W. : Incisural sclerosis and temporal lobe seizures produced by hippocampal herniation at birth. A.M.A. Arch. Neurol. Psychiat. 69: 27, 1963. 
Eisenfeld, A.J., Axelrod, J. and Krakoff, L. : Inhibition of the extraneuronal accumulation and metabol ism of norepinephrine by adrenergic blocking agents. J. Pharmacol. Exp. Ther: 156: $107,1967$.

Elazar, Z., Kado, R.T. and Adey, W.R. : Impedence changes during epileptic seizures. Epilepsia,7: 291, 1966.

Enge1, J., Hanson, L.C.F., Roos, B.E. and Strombergsson, L.E. : Effect of electroshock on dopamine metabolism in rat brain. Psychopharmacologia. (Berl.l 13: 140, 1968.

Esplin, D.W. : Effects of diphenylhydantoin on synaptic transmission in cat spinal cord and stellate ganglion. 3. Pharmacol. Exp. Ther. 120: 301, 1957.

Esplin, D.W. and Curto, E.M. : Effects of trimethadione on synaptic transmission in the spinal cord; antagonism of trimethadione and pentylenetetrazol. J. Pharmacol. Exp. Ther. 121: 457, 1957.

Espl in, D.W. and Freston, J.'1. : Physiological and pharmacological analys is of spinal cord conyulsions. J. Pharmacol. Exp. Ther. 130: 68,1960 .

Esplin, D.W. and Zablocka, B. : Effect of tetanization on transmitter dynamics. Epilepsia 10: 193, 1969.

Essig, C.F.: Possible relation of brain gamma-aminobutyric acid (GABA) to barbiturate abstinence convulsions. Arch. Int. Pharmacodyn. 176: 97, 1968.

Euler, U.S. V. : The presence of the adrenergic neurotransmitter in intraaxonal structures. Acta. Physiol. Scand. 43: 155, 1958.

Euler, U.S. v. : Adrenaline and norepinephrine actions and use in man. Clin. Pharmacol. Ther. 1: 65, 1960.

EuTer, U.S. V. : Some aspects of the mechanisms involved in adrenergic neurotransmission. Perspectives in Biol. and Med. 12: 79, 1968.

Euler, U.S. v. and Gaddum, J.H. : An unidentified depressor substance in certain tissue extract. J. Physiol. 72: 74, 1931.

Euler, U.S. v. and Hillarp, N.-A. : Evidence for the presence of noradrenaline in submicrotropic structures of adrenergic axons. Nature (Lond.) 177: 44, 1956.

Euler, U.S. v. and Lishajko, F. : Effect of some drugs on noradrenal ine release from nerve granules. Biochem. Pharmaco1. 8: 62, 1961. 
EuTer, U.S. V. and Lishajko, F. : Effect of reserpine on the uptake of catecholamines in isolated nerve storage granules. Int. J. Neuropharmacol: 2: 127, 1963.

Euler, U.S. v. and Lishajko, F. : Effect of reserpine on the uptake of catecholamines in adrenergic nerve granules. Acta. Phystol. Scand. 60: $217,1964$.

Euler, U.S. v. and Lishajko, F. : Inhibitory action of adrenergic blocking agents on reuptake and net uptake of noradrenaline in nerve granules. Acta. Physiol. Scand. 74: 501, 1968.

Everett, G.M. and Richards, R.K. : Comparative anticonyulsive action of 3,5,5-trimethyloxazol idine-2,4-dione (tridione), dilantin and phenobarbita1. J. Pharmaco1. Exp. Ther. 81: 402, 1944.

Everett, G.M. and Wigand, R.G. : Central amines and behavioral states: A critique and new data. Proc. Int. Pharmacol. Meeting, First. Stockholm, 8: 85, 1967.

Falck, B. : Observations on the possibilities of the cellular localization of monoamines by a fluorescence method. Acta. Physiol. Scand. 56: Suppl. 197, 1962.

Falck, B., Hillarp, N.-A., Thieme, G. and Torp, A. : Fluorescence of catecholamines and related compounds condensed with formaldehyde. J. Histochem. Cytochem. 10: 348, 1962.

Falck, B. and Owman, C. : A detailed methological description of the fluorescence method for the cellular demonstration of biogenic monoamines. Acta. Univ. Lung. 2 (7) : 1, 1965.

Farrant, J., Harvey, J.A. and Pennefather, J.N.: The influence of phenoxybenzamine on the storage of noradrenaline in rat and cat tissues. Brit. J. Pharmacol. 22: 104, 1964.

Faulkner, T.P. and Mennear, J.H. : Studies on the anticonvulsant action of carbon dioxide in mice. Life Sci. 8: 277, 1969.

Feldberg, $W$. : Synthesis of acetylcholine by tissue of the central nervous system. J. Physiol. 103: 367, 1945.

Feldberg, W. and Sherwood, S.L. : Injections of drugs into the lateral ventricle of the cat. J. Physiol. (Lond.) 123: 148, 1954a.

Feldberg, W. and Sherwood, S.L. : Behavior of cats after intraventricular injections of eserine and DFP. J. Physiol. (Lond.) 125: 488 , 1954b. 
Fessard, A. : Les mecanismes de synchronisation interneuronique et leur intervention dans la crise epileptique. In: Alajouanine, Th. (Ed.) Bases Physiologiques et Aspects $\mathrm{Cl}$ iniques de 1 'Epilepsie. Masson, Paris. 37, 1958.

Fing1, E., 01 sen, L.J., Harding, B.W., Crockett, A.T. and Goodman, L.S. : Effects of chronic anticonvulsant administration upon cortisone-induced brain hyperexcitability. J. Pharmacol. Exp. Ther. 105: 37, 1952.

Fink, G.B. and Swinyard, E.A. : Modification of maximal audiogenic and electroshock seizures in mice by psychopharmacologic drugs. J. Pharmacol. Exp. Ther. 127: 318, 1959.

Fischer, J., Kopin, I.J. and Axe1rod, J. : Evidence for extraneuronal binding of norepinephrine. J. Pharmacol. Exp. Ther. 147: $181,1965$.

Fischer-Williams, M., Poncet, M., Riche, D., and Naquet, R. : Light induced epilepsy in the baboon, Papio Papio : cortical and depth recordings. Electroenceph. Clin. Neurophysiol. 25: 557, 1968.

Fitzerald, J.D. : Perspectives in adrenergic beta-receptor blockade. Cl in. Pharmacol. Ther. 10: 292, 1969.

Foo, J.W., Jowett, A. and Stafford, A. : The effects of some $\beta$-adrenoreceptor blocking drugs on the uptake and release of noradrenaline by the heart. Brit. J. Pharmaco1. 34: 141, 1968.

Frey, H.H. : Note on the interactions of amphetamine with anticonvulsant drugs. Acta. Pharmaco1. (Kbh). 21: 290, 1964.

Friedman, A.H. and Walker, C.A. : Circadian rhythms in rat mid-brain and caudate nucleus biogenic amine levels. J. Physiol. 197: $77,1968$.

Fuller, R.W., Hines, C.W. and Mills, J. : Lowering of brain serotonin level by chloramphetamines. Biochem. Pharmacol. 14: 483, 1965.

Fuller, J.L. and Sjursen, Jr., F.H. : Audiogenic seizures in eleven mouse strains. J. Hered. 58: $135,1967$.

Furchgott, R.F. : Dibenamine blockade in strips of rabbit aorta and its use in differentiating receptors. J. Pharmacol. Exp. Ther. 111: $265,1954$.

Furchgott, R.F. : The pharmacological differentiation of adrenergic receptors. Ann. N.Y. Acad. Sci. 139: 553, 1967. 
Fuxe, K. : Cellular localization of monoamines in the median eminence and the infundibular stem of some mammals. Z. Zellforsch: 61: $710,1964$.

Fuxe, K. : The distribution of monoamine nerve terminals in the central nervous system. Acta. Physio1. Scand. 64: Supp1. 247, 1965a.

Fuxe, K. : Evidence for the existence of monoamine containing neurons in the central nervous system. III. The monoamine nerye terminal. Z. Zellforsch. 65: $573,1965 \mathrm{~b}$.

Fuxe, K., Hokfelt, T. and Nilsson, 0. : Observations on the cellular localization of dopamine in the caudate nucleus of the rat. Z. Zellforsch. 63: 701, 1964.

Gangloff, H. and Monnier, M. : The action of anticonyulsant drugs tested by electrical stimulation of the rabbit cortex, diencephalon and rhinencephalon in the unanesthetized rabbit. Electroenceph. Clin. Neurophysiol. 9: 43, 1967.

Gastaut, H. and Tassinari, C.A. : Triggering mechanisms in epilepsy; the electroclinical point of view. Epilepsia 7: 85, 1966.

Ginsburg, B.E. and Miller, D.S. : Genetic factors in audiogenic seizures. Psychophysiologie, neuropharmacologie de la crise audiogene. Paris: Centre national de la recherche scientifique. 1963.

Glowinski, J. and Axeirod, J. : Effect of drugs on the uptake, release and metabolism of H3-norepinephrine in the rat brain. J. Pharmacol. Exp. Ther. 149: 43, 1965.

Glowinski, J. and Axelrod, J. : Effect of drugs on the disposition of H3-norepinephrine in the rat brain. In : Second Catecholamine Symposium, Milan. 1965. Pharmaco1. Rev. 18: 775, 1966.

Glowinski, J., Axelrod, J. and Iversen, L.L. : Regional studies of catecholamines in the rat brain. IV. Effects of drugs on the disposition and metabolism of H3-dopamine. J. Pharmacol. Exp. Ther. 153: 30, 1966.

Glowinski, J. and Iversen, L.L. : Regional studies of catecholamines in the rat brain. I. The disposition of $(3 \mathrm{H})$ norepinephrine, (3H) dopamine and (3H) Dopa in various regions of the brain. J. Neurochem. 13: 655, 1966.

Goldstein, A. : Biostatistics: An Introductory Text. The Macmillan Co., New York. 184, 1964a. 
Goldstein, A. : Biostatistics: An Introductory Text. The Macmillan Co., New York. 59, 19646.

Goldstein, L. and Munoz, C. : Influence of adrenergic stimulant and blocking drugs on central electrical activity in curarized anima7s. J. Pharmaco1. Exp. Ther. 132: 345, 1961.

Goldstein, M. : Inhibition of norepinephrine biosynthesis at the dopamine- $\beta$-hydroxylation stage. In : Second Catecholamine Symposium, Mi1an. 1965. Pharmaco1. Rev. 18: 77, 1966.

Goldstein, M., Gang, H. and Anagnoste, B. : The inhibition of tyrosine hydroxylase by 4-isopropyl-tropolone. Life Sci. 6: 1457, 1967.

Goodman, L.S., Grewal, M.S., Brown, W.C. and Swinyard, E.A. : Comparison of maximal seizures evoked by pentylenetetrazol (metrazol) and electroshock in mice, and their modification by anticonvulsants. J. Pharmacol. Exp. Ther. 108: 168, 1953.

Goodman, L.S., Toman, J.E.P. and Swinyard, E.A. : The anticonvutsant properties of tridione. Amer. J. Med. I: 213, 1946.

Goodman, L.S., Toman, J.E.P. and Swinyard, E.A. : Anticonvulsant drugs: mechanisms of action and methods of assay. Arch. Int. Pharmacodyn. 78: 144, 1949.

Gray, W.D., Rauh, C.E. and Shanahan, R.W. : Mechanism of the antagonistic action of reserpine on the anticonvulsant effect of inhibitors of carbonic anhydrase. J. Pharmacol. Exp. Ther. 139: 350, 1963.

Green, J.D. : The rhinencephaton and behavior. In : Neurological Basis of Behaviour. Ciba Foundation, London. 222, 1958.

Green, J.D. : The hippocampus. Physio1. Rev. 44: 561, 1965.

Green, J.D. and Adey, W.R. : Electrophysiological studies of hippocampal connections and excitability. Electroenceph. Clìn. Neurophysiol. 8: 245, 1956.

Green, J.D., Clemente, C.D. and DeGrott, J. : Experimental1y induced epilepsy in cat with injury of cornu ammonis. A.M.A. Arch. Neuro1. Psychiat. 78: 259, 1957.

Green, J.D. and Shimamoto, T. : Hippocampal seizures and their propagation. A.M.A. Arch. Neuro1. Psychiat. 70: 687, 1953. 
Gulati, O.D., Gokha7e, S.D., Parikh, H.M., Udwadia, B.P., and Krishnamurty, V.S.R. : Evidence for a sympathetic aipha receptor blocking action of beta receptor blocking agents. J. Pharmacol. Exp. Ther. 166: 35, 1969.

Gutman, Y. and Weil-Malherbe, H. : The intracellular distribution of brain catecholamines. J. Neurochem. 14: 619, 1967.

Haefely, W. and Hurlimann, A. : Substance P, a highly active naturally occurring polypeptide. Experientia. (Base1) 18: 297, 1962.

Haggendal, J. and Lindqvist, M. : Behavior and monoamine levels during long-term administration of reserpine to rabbits. Arch. Physiol. Scand. 57: 437, 1963.

Haggendal, J. and Lindqvist, M. : Brain monoamine levels and behavior during long-term administration of reserpine. Int. J. Neuropharmaco1. $\underline{3}: 59,1964$.

Hall, G.E. : Physiological studies in experimental insul in and metrazol shock; composite prel iminary study by members of Department of Medical Research, Banting Institute, University of Toronto. Amer. J. Psychiat. 95: 553, 1938.

Hanigan, W.C., Scudder, C.L. and Karczmar, A.G. : Adrenergic, serotoninergic and cholinergic systems and electroconvulsive seizures in mice. Fed. Proc. 29: 1360, 1970.

Hanson, L.C.F. : The disruption of conditioned avoidance response following selective depletion of brain catecholamines. Psychopharmacologia (Berl.). $\underline{6}:$ 100, 1965.

Harvey, S.C. and Nickerson, M. : Reactions of dibenamine and some congeners with substances of biological interest in relation to the mechanism of adrenergic blockade. J. Pharmacol. Exp. Ther. 112: $174,1954$.

Havlicek, V. : The effect of dl-3,4-dihydroxyphenyl serine (precursor of norepinephrine) on the ECOG of unres trained rats. Int. J. Neuropharmacol. $\underline{6}: 83,1967$.

Hayashi, T.A. : A physiological study of epileptic seizures following cortical stimulation in animals and its application to human clinics. Jap. J. Physiol. 3: 46, 1952.

Hayashi, T.A. and Nagai, K. : Action of omega-amino acids on the motor cortex of higher animals especially gammaamino-beta-oxy-butyric acid as the real inhibitory principle in brain. Abstr. Commun. XX. Int. Physiol. Congr. 470, 1956. 
Hertting, G., Axelrod, 3. and Whitby, L.G. : Effect of drugs on the uptake and metabol ism of H3-norepinephrine. J. Pharmacol. Exp. Ther: 134: 146, 1961 .

Hess, S.M., Connamacher, R.H., Ozaki, M. and Udenfriend, S. : The effects of $\alpha$-methyl-meta-tyrosine on the metabolism of norepinephrine and serotonin in vivo. J. Pharmacol. Exp. Ther. 134: $129,1961$.

Hillarp, N.-A., Fuxe, K. and Dahlstrom, A. : Demonstration and mapping of central neurons containing dopamine, noradrenal ine and 5-hy-. droxytryptamine and their reactions to psychopharmaca. In : Second Catecholamine Symposium, Milan. 1965. Pharmacol. Rey. 18: 727,1966 .

Hillarp, N.-A. and Malmfors, T. : Reserpine and cocaine blocking of the uptake and storage mechanisms in adrenergic nerves. Life Sci. 3: 703, 1964.

Himwich, H.E., Essig, C.F., Hampson, J.L. and Bales, P.D. : Effect of trimethadione (tridione) and other drugs on convulsions caused by di-isopropyl fluorophosphate (DFP). Amer. J. Psychiat. 106: $816,1950$.

Holtz, P. : Role of L-Dopa decarboxylase in the biosynthesis of catecholamines in nervous tissue and the adrenal medulla. Pharmacol. Rev. 11: 317, 1959.

Holtzbauer, M. and Vogt, M. : Depression by reserpine of the noradrenal ine concentration in the hypothalamus of the cat. $\mathrm{J}$. Neurochem. 1: 8, 1956.

Hornykiewicz, 0. : Dopamine (3-hydroxytyramine) and brain function. In : Second Catecholamine Symposium, Milan. 1965. Pharmacol. Rev. 18: 925, 1966.

Innes, I.R. and Nickerson; M. : Drugs acting on postganglionic adrenergic nerve endings and structures innervated by them (sympathomimetic drugs). In : Goodman, L.S. and Gilman, A. (Ed.) The Pharmacological Basis of Therapeutics. The Macmillan Co., New York. 3rd. ed.: 477, 1965.

Iversen, L.L. : Inhibition of noradrenal ine uptake by sympathomimetic amines. J. Pharm. Pharmacol. 16: 435, 1964.

Iversen, L.L. : Inhibition of noradrenal ine uptake by drugs. J. Pharm. Pharmacol. 17: 62, 1965. 
Iversen, L.L. and Glowinski, J. : Regional studies of catecholamines in the rat brain. II. Rate of turnover of catecholamines in various brain regions. J. Neurochem. 13: 671, 1966.

Iversen, L.L., Glowinski, J. and Axelrod, J. : The uptake and storage of H3-norepinephrine in the reserpinized rat heart. J. Pharmacol. Exp. Ther. 150: 173, 1965.

Izquierdo, J.A., Jofre, I.J. and Dezza, M.A. : Effect of pyrogallol on the catecholamine content of cortex, diencephalon, mesencephalon, rhombencephalon and cerebellum of mouse and rat. Med. Exp. (Basel) 10: $45,1964$.

Jasper, H. and Erickson, T.C. : Cerebral blood flow and pH in excessive cortical discharge induced by metrazol and electrical stimulation. J. Neurophysiot. $4: 333,1941$.

Jenney, E.H. : Changes in convulsant thresholds after rauwolfia serpentina, reserpine and veriloid. Fed. Proc. 13: 370, 1954.

Jonason, J., Rosengren, E. and Waldeck, B. : Effects of some pharmacologically active amines on the uptake of arylalkylamines ..by adrenal medullary granules. Acta. Physiol. Scand. 60: 136, 1964.

Jonason, J. and Rutledge, C.0. : Effects of reserpine, dopamine and nialamid on the synthesis of $\alpha$-methylnoradrenaline. Europ. $J$. Pharmaco1. 6: 24, 1969.

Jones, B.J. and Roberts, D.J. : The effects of intracerebroyentricularly administered noradnamine and other sympathomimetic amines upon leptazol convulsions in mice. Brit. J. Pharmacol. 34: 27, 1968.

Judah, J.D. and Ahmed, K. : The biochemistry of sodium transport. Biol. Rev. 39: 160, 1964.

Kaada, B.R. : Somato-motor, autonomic and electrocorticographic responses to electrical stimulation of "rhinencephalic" and other structures in primates, cat and dog. Acta. Physiol. Scand. 24: Suppl. 83, 1951.

Keith, H.M. : Factors influencing experimentally produced convulsions. Arch. Neurol. Psychiat. 29: 148, 1933.

Kety, S.S., Javoy, F., Thierry, A.M., Julou, L. and Glowinski, J. : A sustained effect of electroconvulsive shock on the turnover of norepinephrine in the nervous system of the rat. Proc. Nat. Acad. Sci. (Wash.) 58: 1249, 1967. 
Killam, Jr., K.F. : Convulsant hydrazides. II. Comparison of electrical changes and enzyme inhibition induced by the administration of thiosemicarbazide. J. Pharmacot. Exp. Ther. 119: 263, 1957.

Killam, Jr., K.F. : Action of anticonvulsant drugs in the baboon (Papio Papio). Fed. Proc. 26: 763, 1967.

Killam, Jr., K.F. : Genetic models of epilepsy with special reference to the syndrome of the Papio Papio. Epilepsia 10: 229, 1969.

Killam, Jr., K.F., Killam, E.K. and Naquet, R. : An animal model of light sensitive epilepsy. Electroenceph. Clin. Neurophysiol. 22: $497,1967$.

Kirshner, N. : Uptake of catecholamines by a particulate fraction of the adrenal medu11a. Science. 135: 107, 1962.

Kirshner, N., Rorie, M. and Kamin, D. : Inhibition of dopamine uptake in yitro by reserpine administered in yiyo. J. Pharmacol. Exp. Ther. 141: 285, 1963.

Kluver, H. and Barrera, E. : Method for combined staining of cells and fibers in nervous system. J. Neuropath. and Exp. Neurol. 12: $400,1953$.

Kobinger, W. : Beeinflussung der cardiazolk rampfschwelle durch veranderten 5-hydroxytryptamingehalt des zentralnervensystems. Arch. Exp. Path. Pharmako1. 233: 559, 1958.

Koe, B.K. and Weissman, A. : p-Chlorophenylalanine: A specific depletor of brain serotonin. J. Pharmacol. Exp. Ther. 154: $499,1967$.

Koelle, G.B. : The histochemical localization of cholinesterases in the central nervous system of the rat. J. Comp. Neurol: 160: $211,1954$.

Koelle, G.B. : Neurohumoral transmission and the autonomic nervous system. In : Goodman, L.S. and Gilman, A. (Ed.) The Pharmacological Basis of Therapeutics. The Macmillan Co., New York. 3rd..ed.: 399, 1965.

Kohli, R.P. and Kishor, K. : The anticonvulsant activity of hydroxylamine. Arch. Int. Pharmacodyn. 154: 89, 1965.

Kohli, J.D. and Ling, G.M. : Alpha-adrenergic blocking action of proprano101. J. Pharm. Pharmaco1. 19: 629, 1967. 
Konig, J.F.R. and Klippel, R.A. : The Rat Brain. A Stereotaxic Atlas of the Forebrain and lower Parts of the Brainstem. Will jams and Wilkins Co., Baltimore. 1963.

Kopeloff, L.M., Chusid, J.G. and Kopeloff, N. : Chronic experimental epilepsy in Macaca mulatta. Neurology (Minneap.) 4: 218, 1954.

Kopin, I.J. : Storage and metabolism of catecholamines; the role of monoamine oxidase. Pharmacol. Rev. 16: 179, 1964.

Kreindler, A. : Experimental Epilepsy. Prog. Brain Res. 19: 1965.

Kuriyama, K., Roberts, E. and Rubinstein, M.K. : Elevation of $\gamma$ aminobutyric acid in brain with amino-oxyacetic acid and susceptibility to convulsive seizures in mice. A quantitative reevaluation. Biochem. Pharmaco1. 15: 221, 1966.

Landisch, W., Steinhauff, N. and Matussek, N. : Chronic administration of electroconsul sive shock and norepinephrine metabolism in the rat brain. II. 7-H3-NE metabol ism after intracisternal injection with and without the influence of drugs in different brain regions and by 7-H3-NE uptake in vitro. Psychopharmacologia (Ber1.) 15: 196, 1969.

Lembeck, F. and Zetler, G. : Sunstance P : a polypeptide of possible physiological significance, especially within the nervous system. In: Pfeiffer, C.G. and Smythies, J.R. (Ed.) Int. Rev. Neurobiol. Acad. Press, New York. 4: 159, 1962.

Lehman, A. : Augiogenic seizure data in mice supporting new theories of biogenic amine mechanisms in the central nervous system. Life Sci. 6: 1423, 1967.

Lessin, A.W. and Parkes, M.W. : The effects of reserpine and other agents upon leptazol convulsions in mice. Brit. J. Pharmacol. 14: 108, 1959.

Leszkovszky, G. and Tardos, L. : Some effects of propranolol on the central nervous system. J. Pharm. Pharmacol. 17: 518, 1965.

Li, C.L. and Chou, J.N. : Inhibitory interneurons. In : Inhibition of the Nervous System and $\gamma$-aminobutyric Acid. Pergamon Press, New York. 147, 1960.

Liberson, W.T. and Cadilhac, J.D. : Further studies of hippocampal seizure states. Electroenceph. Clin. Neurophysiol. I: Suppl. 3, 1953. 
Lish, P.M., Weikel, J.W. and Dungan, K.W. : Pharmacological and toxicological properties of two new $\beta$-receptor antagonists. J. Pharmacol. Exp. Ther. 149: 161, 1965.

Litchfield, J. and Wilcoxon, F. : A simplified method of evaluating dose-effect experiments. J. Pharmaco1. Exp. Ther. 96: 99, 1949.

Longo, V.G. : Behavioral and electroencephalographic effects of atropine and related compounds. Pharmaco7. Rev. 18: 965, 1966.

Longo, V.G. and Silvestrini, B. : Effects of adrenergic and cholinergic drugs injected by intra-carotid route on electrical activity of brain. Proc. Soc. Exp. Biol. (New York). 95: 43, 1957.

Lundborg, P. : Storage function and amine levels of the adrenal medullary granules at various intervals after reserpine treatment. Experientia (Base1) 19: 479, 1963.

Lundborg, P. and Stitzel, R.E. : Studies on the relationship between adrenergic nerve function and granular uptake mechanisms. Brit. J. Pharmacol. 33: 98, 1968.

Lyons, L.B., Williams, H.L. and Arnold, E.A. : The pyridoxine-deficient state in two strains of inbred mice. J. Nutr. 66: 261, 1958.

MacLean, P.D. : Chemical and electrical stimulation of hippocampus: in unanes thetized animals. I. Methods and electroencephalographic findings. A.M.A. Arch. Neurol. Psychiat. 78: 113, 1957a.

MacLean, P.D. : Chemical and electrical stimulation of hippocampus in unanesthetized animals. II. Behavioral Findings. A.M.A. Arch. Neurol. Psychiat. 78: 128, 1957b.

Maickel, R.P., Cox, Jr., R.H., Saillant, J. and Miller, F.P. : A method for the determination of serotonin and norepinephrine in discrete areas of rat brain. Int. J. Neuropharmacol. 7: 275, 1968.

Maitre, L. : Effects of long-term administration of pyrogal101 on tissue catecholamine levels, monoamine oxidase and catechol-omethyl-transferase activities in the rat. Biochem. Pharmacol. 15: $1935,1966$.

Malcolm, J.L., Saraiva, P. and Spear, P.J. : Cholinergic and adrenergic inhibition in the rat cerebral cortex. Int. J. Neuropharmacol. 6: $509,1967$. 
Malmfors, T. : Studies on adrenergic nerves: The use of rat and mouse iris for direct observations on their physiology and pharmacology at cellular and subcellular level's. Acta. Physiol. Scand. 64: SuppT. 248, 1965.

Manshardt, J. and Wurtman, R.T. : Daity rhythm in the noradrenaline content of rat hypothalamus. Nature (Lond.) 217: 574, 1968.

Marley, E. : Behavioral and electrophysiological effects of catecholamines. In : Second Catecholamine Symposium, Milan. 1965. Pharmacol. Rev. 18: 753, 1966.

Matsuoka, M., Yoshida, H. and Imaizumi, R. : Effect of pyrogallol on the catecholamine content of the rabbit brain. Biochem. Pharmacol. 11: 1109, 1962.

Maynert, E.W. : The role of biochemical and neurohumoral factors in the laboratory evaluation of antiepileptic drugs. Epilepsia 10: 145,1969 .

Maynert, E.W. and Kaji, H. : On the relationship of brain $\gamma$-aminobutyric acid to convulsions. J. PharmacoT. Exp. Ther: 137: $114,1962$.

Maynert, E.N. and Kuriyama, K. : Some observations on nerye-ending particles and synaptic vesicles. Life Sct. 3: 1067, 1964.

Maynert, E.W. and Levi, R. : Stress-induced release of brain norepinephrine and its inhibition by drugs. J. Pharmacol. Exp. Ther. 143: $90,1964$.

McGeer, E.G., Ikeda, H., Asakura, T. and Wada, U.A. : Lack of abnormality in brain aromatic amines in rats and mice susceptible to audiogen ic seizures. J. Neurochem. 16: 945, 1969.

McGeer, E.G., McGeer, P.L. and Peters, D.A. : Inhibition of brain tyrosine hydroxylase by 5-halotryptophane. Life Sci. 6: 2221, 1967.

McIlwain, H. : Biochemistry and the Central Nervous System. J. \& A. Churchi11, London. 1955.

McKhann, G.M. and Tower, D.B. : Gamma-aminobutyric acid: a substrate for oxidative metabolism of cerebral cortex. Amer. J. Physiol. 196: $36,1959$.

McLennan, H. and Elliott, K.A.C. : Effects of convulsant and narcotic drugs on acetylchol ine synthesis. J. Pharmacol. Exp. Ther, 103: 35,1951 . 
Mennear, J.H. and Rudzik, A.D. : Potentiation of the anticonvulsant action of acetazolamide. J. Pharm. Pharmacol. 18: 833, 1966.

Mennear, J.H. and Rudzik, A.D. : The effect of pronethalol on the anticonvulsant action of acetazolamide. Life Sci. 7: 1265, 1968.

Mercier, J. : Note preliminaire sur i'-influence exercee par quelque medicaments sympathicometique sur la crise audiogene du rat albinos. C.R. Soc. Biol. (Paris) 143: 1125, 1949.

Mercer, E.N. and Osborn, J.A. : The current status of diphenylhydantoin in heart disease. Ann. Int. Med. 67: 1084, 1967.

Merritt, H.H. and Putnum, T.J. : A new series of anticonvulsant drugs tested by experiments on animals. Arch. Neurol. Psychiat. (Chicago) 39: 1003, 1938.

Millichap, J.G. : Effects of drugs on experimental febrile seizures: development of a new specific therapy. Amer. J. Dis. Child. 96: $602,1958$.

Millichap, J.G. : Anticonvulsant Drugs. In : Physiological Pharmacology. Acad. Press, New York. II: 97, 1965.

Millichap, J.G., Hernandez, P., Zales, M.R., Halpern, L.A. and Kramer, B.I. : Studies in febrile seizures. Part 4. Evaluation of drug effects and development of a potential new therapy (Pyrictal). Neurology (Minneap.) 10: 575, 1960.

Minz, B., Albe-Fessard, D. and Buser, P. : A propos de l'epilepsie corticale provoquee chez le lapin thyreoprive chronique. J. Physiol. (Paris) 43: 815, 1951.

Minz, B. and Domino, E.F. : Effect of epinephrine and norepinephrine on electrically induced seizures. J. Pharmacol. Exp. Ther.. 107: $204,1953$.

Montagu, K.A. : Catechol compounds in rat tissue and in brains of different anima1s. Nature (Lond.) 180: 244, 1957.

Moore, K.E. : Effects of $\alpha$-methyltyrosine on brain catecholamines and conditioned behavior in guinea pigs. Life Sci. 5: 55, 1966.

Moore, K.E. and Lariviere : Effects of stress and D-amphetamine on rat brain catecholamines. Biochem. Pharmaco1: 13: 1098, 1964. 
Moore, K.E. and Rech, R.H. : Antagonism by monoamine oxidase inhibitors of a -methyl tyrosine-induced catecholamine depletion and behavioral depression. J. Pharmacol. Exp. Ther. 156: 70, 1967.

Moore, K.E., Wright, P.F. and Bert, J.K. : Toxicologic studies with $\alpha$-methyltyrosine, an inhibitor of tyrosine hydroxylase. J. Pharmaco1. Exp. Ther. 155: 506, 1967.

Morales-Aguiers, A. and Vaughan-Williams, E.M. : The effects on cardiac muscle of $\alpha$-receptor antagonists in relation to their activity as local anesthetics. Brit. J. Pharmacol. 24: 332, 1965.

Moran, N.C. : The deyelopment of beta adrenergic blocking drugs. A retrospective and prospective evaluation. Ann. N.Y. Acad. Sci. 139: $649,1967$.

Moreira, M.G. and Osswald, W. : Pronethalo1-induced reyersal of adrenergic vasodepression. Nature. 208: 1006, 1965.

Morrell, F., Bradley, W. and Ptashne, M. : Effects of drugs on discharge characteristics of chronic epileptogenic lesions. Neurology (Minneap.) 9: 492, 1959.

Murmann, W., Almirante, L. and Saccani-Guelfi, M. : Central nervous system effects of four $\beta$-adrenergic receptor blocking agents. J. Pharm. Pharmacol. 18: $317,1966$.

Musacchio, J., Julou, L., Ketey, S. and Glowinski, J. : Increase in rat brain tyrosine hydroxylase activity produced by electroconvulsive shock. Nat. Acad. Sci. 63: 1117, 1969.

Nagatsu, T., Levitt, M. and Udenfriend, S. : Tyrosine hydroxylase. The initial step in norepinephrine biosynthesis. J. Biol. Chem. 239: $2910,1964$.

Nickerson, M. : Drugs inhibiting adrenergic nerves and structures inneryated by them. In : Goodman, L.S. and Gilman. A. (Ed.) The Pharmacological Basis of Therapeutics. The Macmillan Co., New York. 3rd. ed. : 546, 1965.

Nickerson, M. : New developments in adrenergic blocking drugs. Ann. N.Y. Acad. Sci. 139: 571, 1967.

Nickerson, M., Goodman, L.S. and Nomaguchi, G. : Pharmacological properties of a new adrenergic blocking agent; N, N-dibenzìl- $\beta$ chloro-ethylamine (dibenamine). J. Pharmacol. Exp. Ther. 89: 167, 1947. 
Nickerson, M. and Gump, W.S. : The chemical basis of adrenergic Blocking activity in compounds related to dibenamine. J. Pharmacol. Exp. Ther. 97: 25, 1949.

Notkin, J. and Pike, F.H. : Some experiments on effects of caffeine, adrenal in and bromides upon susceptibility to experimentally induced convulsions in animals. Amer. J. Psychiat. 10: 771, 1931.

Nyback, H., Sedval1, G. and Kopin, I.J. : Accelerated synthes is of dopamine-Cl4 from tyrosine-C14 in rat brain after chlorpromazine. Life Sci. 6: 2307, 1967.

Paasonen, M.K. and Vogt, M. : The effect of drugs on the amounts of substance $P$ and 5-hydroxytryptamine in mammalian brain. J. Physiol. 131: $617,1956$.

Patil, P.N. : Steric aspects of adrenergic drugs. VIII. Optical isomers of beta adrenergic receptor antagonists. J. Pharmacol. Exp. Ther. 160: $308,1968$.

Penfield, W. and Erickson, T.C. : Epilepsy and Cerebral Localization. C.C. Thomas, Springfield, I11. 1941.

Pfeifer, A.K. and Galambos, E. : Action of alpha methyl Dopa on the pharmacologic and biochemical effect of reserpine in rats and mice. Biochem. Pharmacol. 14: 37, 1965.

Pfeifer, A.K. and Galambos, E. : The effect of $(+) p$-chloroamphetamine on the susceptibility to seizures and on the monoamine level in brain and heart of mice and rats. J. Pharm. Pharmacol. 19: 400 1967.

Phillips, C.G. : Intracellular records from betz cells in the cat. Quart. J. Exp. Physiol. 41: 58, 1956.

Phillis, J.W. and York, D.H. : An intracortical chol inergic inhibitory synapse. Life Sci. I: 65, 1968.

Pletscher, A. : Release of 5-hydroxytryptamine by benzoquinolizine derivatives with sedative action. Science. 126: 507, 1957a.

Pletscher, A. : Wirkung von isopropyl-isonikotinsaurehydrazid auf den stoffwechsel von ketacholaminen und 5-hydroxytryptamin im gehirn. Schweiz. Med. Wecher. 87: 1532, $1957 \mathrm{~b}$.

Pletscher, A., Basendorf, H. and Bachtold, H.P. : Benz(a)chinolizine, eine neue korperklasse mit wirkung auf den 5-hydroxytryptaminund noradrenalin-stoffwechsel des gehirns. Arch. Exp. Path. Pharmak. 232: 499, 1958. 
Pletscher, A., Shore, P.A. and Brodie, B.B, : Release of brain seroton in by reserpine. J. Pharmaco1. Exp. Ther. 116: 46, 1956.

Plotnikoff, N.P. : Bioassay of potential tranquillizers and sedatives against audiogenic seizures in mice. Arch. Int. Pharmacodyn. 116: $130,1958$.

Plotnikoff, N.P. and Green, D.M. : Bioassay of potential ataraxic agents againat seizures in mice. J. Pharmacol. Exp. Ther: 119: 294, 1957.

Porter, C.C., Totaro, J.A. and Leiby, C.M. : Some biochemical effects of $\alpha$-methyl-3,4-dihydroxyphenyl-alanine and related compounds in mice. J. Pharmacol. Exp. Ther. 134: 139, 1961.

Porter, C.C., Toraro, J.A., Burcin, A. and Wynosky, E.R. : The effect of the optical isomers of alpha-methyl-p-tyrosine upon brain and heart catecholamines in the mouse. Biochem. Pharmacol. 15: 583, 1966.

Prockop, D.J., Shore, P.A. and Brodie, B.B. : Anticonvul sant properties of monoamine oxidase inhibitors. Ann. N.Y. Acad. Sci. 80: 643, 1959a.

Prockop, D.J., Shore, P.A. and Brodie,B.B. : An anticonvulsant effect of monoamine oxidase inhibitors. Experientia. 15: 145, 1959b.

Putnum, T.J. and Merritt, H.H. : Experimental determination of the anticonvulsant properties of some phenyl derivatives. Science. 85: $525,1937$.

Ranck, Jr., J.B. : Electrical impedance in the subicular area of rats during paradoxical sleep. Exp. Neurol. 16: 416, 1946.

Raper, C. and Wale, J. : Specificity of $\beta$-receptor antagonists. Europ. J. Pharmacol. $3: 279,1968$.

Rech, R.H., Borys, H.K. and Moore, K.E. : Alterations in behavior and brain catecholamine levels in rats treated with $\alpha$-methyltyrosine. J. Pharmaco1. Exp. Ther. 153: 412, 1966a.

Rech, R.H., Borys, H.K. and Moore, K.E. : Behavior and brain catecholamine levels in rats treated with $\alpha$-methyl tyrosine $(\alpha-m T)$. Pharmacologist. 8: 214, 1966b.

Rech, R.H., Carr, L.A. and Moore, K.E. : Behavior and brain catecholamines after $\alpha$-methyl-tyrosine $(\alpha-m T)$ and other central depressant drugs. Pharmacologist. $\underline{9}: 213,1967$. 
Reis, D.J., Weinbren, M. and Corvel1i, A. : A circadian rhythm of norepinephrine regionally in cat brain, its relationship to environmental lighting and to egional diurnal variations in brain serotonin. J. Pharmacol. Exp. Ther. 164: 135, 1968.

Richards, R.K. and Everett, G.M. : Tridione : a new anticonvulsant drug. J. Lab. Clin. Med. 31: 1330, 1946.

Richards, R.K. and Perlstein, M.A. : Tridione, a new drug for the treatment of convulsive and related disorders. Arch. Neurol. Psychiat. (Chícago) 55: 164, 1946.

Roelofs, G.A. : Behavioral state and seizure susceptibility - a study of responses upon electrical stimulation of the basal temporal lobe in cats under different behavioral conditions. Acta. Neurol. Scand. 43: Supp1. 26, 1967.

Roldan, E., Weiss, T. and Fifkora, E. : Excitability changes during sleep cycle of the rat. Electroenceph. Clin. Neurophysiol. 15: $775,1963$.

Rosenblueth, A. and Cannon, W.B. : Cortical responses to electrical stimulation. Amer. J. Physiol. 135: 690, 1942.

Roth, R.H. and Stone, E.A.: The action of reserpine on noradrenaline biosynthesis in sympathetic nerve tissue. Biochem. Pharmacol. 17: 1581, 1968.

Rudzik, A.D. and Mennear, J.H. : Aatagonism of anticonvulsants by adrenergic blocking agents. Proc. Soc. Exp. Biol. Med. 122: 278,1966 .

Rudzik, A.D. and Mennear, J.H. : The mechanism of action of anticonvulsants. II. Acetazolamide. Life Sci. $\underline{5}: 747,1966 \mathrm{~b}$.

Ruf, H. : Experimentelle untersuchungen durch sauerstoff und adrenalin. Arch. f. Psychiatr. u.z. Nervenkr. 187: 97, 1951.

Rutledge, C.0. and Weiner, N. : The effect of reserpine upon the synthesis of norepinephrine in the isolated rabbit heart. $J$. Pharmaco1. Exp. Ther. 157: 290, 1967.

Saelens, J.K., Schoen, M.S. and Kovacsics, G.B. : An enzyme assay for norepinephrine in brain tissue. Biochem. Pharmacol: 16: 1043, 1967.

Sansone, M. and Del1'Omodarme, G. : Influence de 1 'isocarboxazide (Marplan) et de deux nouveaux inhibiteurs de la MAO sur Tes convulsions clonique et sur 1a mortalite par metrazol. Arch. Int. Pharmacodyn. 144: 392, 1963. 
Săno, I., Gamo, T., Kakimoto, Y., Taniguchi, K., Takesada, M. and Nishinuma, K. : Distribution of catechol : compounds in human brain. Acta, Biochem, Biophysic. 32: 586, 1959.

Satoh, T., Iwamoto, T. and Tokumitsu, $Y_{0}$ : Effects of tranquilizing agents in brain noradrenaline and dopamine levels of shocked rats. Jap. J. Pharmaco1. 14: 63, 1964.

Schallek, W. and Kuehn, A. : Effect of trimethadione, diphenylhydantoin and chlordiazepoxide on after discharges in brain of cat. Proc. Soc. Exp. Biol. Med. 112: 813, 1963.

Schapiro, S. : Effect of catecholamine blocking agent (dibenzyline) on organ content and urine excretion of noradrenaline and adrenaline. Acta. Physiol. Scand. 42: 371, 1958.

Scheving, L.E., Harrison, W.H., Gordon, P. and Pauly, J.E. : Daily fluctuation (Circadian and ultradian) in biogenic amines in rat brain. Amer. J. Physio1. 214: 166, 1968.

Schlesinger, K., Boggan, W. and Freedman, D.X. : Genetics of audiogenic seizures. I. Relation to brain serotonin and norepinephrine in mice. Life Sci. 4: 2345, 1965.

Schlesinger, K., Boggan, W. and Freedman, D.X. : Genetics of audiogenic seizures. II. Effects of pharmacological manipulations of brain serotonin, norepinephrine and gamma-aminobutyric acid. Life Sci. 7: 437, 1968a.

Schlesinger, K., Boggan, W. and Griek, B.J. : Pharmacogenetic correlates of pentylenetetrazol and electroconvulsive seizure thresholds in mice. Psychopharmacologia (Ber1.) 13: 181, 1968b.

Schlesinger, K., Elston, R.C. and Boggan, W. : The genetics of soundinduced seizures in inbred mice. Genetics. 54: 95, 1966.

Schlesinger, K., Stavnes, K.L. and Boggan, W. : Modification of audiogenic and pentylenetetrazol seizures with gamma-aminobutyric acid, norepinephrine and serotonin. Psychopharmacologia. 15: 226, 1969.

Schmidt, R.P., Thomas, L.B. and Ward, Jr., A.A. : The hyperexcitable neurone. Microelectrode studies of chronic epileptic foci in monkey. J. Neurophysiol. 22: 285, 1959.

Scudder, C.L., Karczmar, A.G., Everett, G.M., Gibson, J.E. and Rifkin, M. : Brain catecholamines and serotonin levels in various strains and genera of mice and a possible interpretation for the correlations of amine levels with electroshock latency and behavior. Int. $\mathrm{J}$. Neurophanmaco1. 5: 343, 1966. 
Sedvall, G. : Short term effects of reserpine on noradrenaline levels in skeletal muscle, Acta. Physiol. Scand. 62: 101, 1964.

Sedva11, G. and Thorson, J. : Adrenergic transmission at vasoconstrictor nerve terminal.s partially depleted of noradrenaline. Acta. Physiol. Scand. 64: 251, 1965.

Shore, P.A. : Recent biochemical developments with monoamine oxidase inhibitors. Dis. Nerv. Syst. 21: Supp1. 62-3, 1960.

Shore, P.A. : The mechanism of norepinephrine depletion by reserpine, metaraminol and related agents. The role of monoamine oxidase. In : Second Catecholamine Symposium, Milan. 1965. Pharmacol. Rey. 18: $561,1966$.

Skou, J.C. : Enzymatic basis for active transport of $\mathrm{Na}^{+}$and $\mathrm{K}^{+}$across cell membranes. Physiol. Rev. 45: 596, 1965.

Somani, P. and Lum, B.K. : The antiarrhythmic actions of beta adrenergic blocking agents. J. Pharmacol. Exp. Ther. 147: 194, 1965.

Sourkes, T.L. : Dopa decarboxylase : substrate, coenzyme, inhibitors. In : Second Catecholamine Symposium, Milan. 1965. Pharmacol. Rev. 18:53, 1966 .

Spector, S., Sjoerdsma, A. and Udenfriend, S. : Blockade of endogenous norepinephrine synthes is by $\alpha$-methyl-tyrosine, an inhibitor of tyrosine hydroxylase. J. Pharmacol. Exp. Ther. 147: 86, 1965.

Spoerlein, M.T. and Ellman, A.M. : Facilitation of metrazol-induced seizures by iproniazid and beta phenylisopropylhydrazine in mice. Arch. Int. Pharmacodyn. 133: 193, 1961.

Stjarne, L., Roth, R.H. and Lishajko, E. : Noradrenaline formation from dopamine in isolated subcellular particles from bovine splenic nerve. Biochem. Pharmacol. 16: 1729, 1967.

Stone, W.E., Webster, J.E. and Gurdjan, W.S. : Chemical changes in the cerebral cortex associated with convulsive activity. J. Neurophysio1. 8: $233,1945$.

Streeter, E.C.: A note on the history of the convulsive state prior to Boerhaave. Res. Publ. A. Nerv. and Ment. Dis. 7: 5, 1931.

Strobos, R.R.J. and Spudis, E.V. : Effect of anticonvulsant drugs on cortical and subcortical seizure discharges in cats. Arch. Neurol. 2: $399,1960$. 
Swinyard, E.A. : Laboratory assay of clinically effective antiepileptic drugs. J. Amer. Pharm. Assoc., Sci. Ed. 38: 201, 1949.

Swinyard, E.A. : Laboratory eyaluation of antiepileptic drugs, Epilepsia. 10: 107,1969 .

Swinyard, E.A., Boson, F.C.B. and Goodman, L.S. : Effect of epinephrine and norepinephrine on excitability of central nervous system of mice. J. Pharmacol. Exp. Ther. 144: 52, 1964.

Swinyard, E.A., Brown, W.C. and Goodman, L.S. : Comparative assays of antiepileptic drugs in mice and rats. J. Pharmacol. Exp. Ther. 106: $319,1952$.

Swinyard, E.A., Radhakrishan, N. and Goodman, L.S. : Effect of brief restraint on the convulsive threshold of mice. J. Pharmacol. Exp. Ther. 138:337, 1962.

Swinyard, E.A., Toman, J.E.P. and Goodman, L.S. : The effect of cellular hydration on experimental electroshock convulsions. J. Neurophysiol.9: $47,1946$.

Tainter, M.L., Tainter, E.G., Lawrence, W.S., Neuru, E.N., Lackey, R.W., Luduena, F.P., Kirtland, H.B. and Gonzales, R.I. : Influence of various drugs on the threshold for electrical convulsions. J. Pharmacol. Exp. Ther. 79: 42, 1943.

Tani, M. : Convulsions induced by the intracarotid injection of $\mathrm{Na}$ glutamate and aspartate. J. Physiol. Soc. (Japan) 16: 685, 1954.

Tapia, R. and Awapara, J. : Formation of $\gamma$-aminobutyric acid (GABA) in brain of mice treated with L-glutamic acid- $\gamma$-hydrazide and pyridoxal phosphate- $\gamma$-glutamyl hydrazone. Proc. Soc. Exp. Biol. Med. 126: 218, 1967.

Taverner, D. : The action of local anesthetics on the spinal cord of the cat. Brit. J. Pharmacol. 15: 201, 1960.

Taylor, Jr., R.J., Stubbs, Jr., C.S. and El lenbogen, L. : Tyrosine hydroxylase inhibition in vitro and in vivo by chelating agents. Biochem. Pharmacol. 18: 587, 1969.

Temkin, 0. : The Falling Sickness. Johns Hopkins Press, Baltimore. 1945.

Toman, J.E.P. : Neuropharmacology of peripheral nerve. Pharmacol. Rey. 4: $168,1952$. 
Toman, J.E.P. : Drugs effective in convulsive disorders. In: Goodman, L.S. and Gilman, A. (Ed.) The Pharmacological Basis of Therapeutics. The Macmillan Co., New York. 3rd. ed.: 215, 1965.

Toman, J.E.P. and Goodman, L.S. : Anticonvulsants. Physiol. Rev. 28: $409,1948$.

Toman, J.E.P., Swinyard, E.A. and Goodman, L.S. : Properties of maximal seizures and their al teration by anticonvulsant drugs and other agents. J. Neurophysio1. 9: 231, 1946.

Torchiana, M.L., Herbert, C.W., Stavroski, J., Ludden, C.T., Stone, C.A. : Effect of methyldopa and related agents on pressor responses to tyramine in reserpine-pretreated rats and dogs. J. Pharmaco1. Exp. Ther. 151: 242, 1966.

Tower, D.B. : The evidence for a neurochemical basis of seizures. In : Baldwin, M. and Bailey, P. (Ed.) Temporal Lobe Epilepsy. Thomas, Springfield, I11. 301, 1958.

Tower, D.B. : Neurochemistry of Epilepsy. Thomas, Springfield, I11. 1960.

Turner, T.A.R. and Spencer, P.S.J. : Effect of pretreatment with monoamine oxidase inhibitors or (+)-amphetamine on leptazol convulsions in mice and rats. J. Pharm. Pharmacol. 20: Suppl. 122S, 1968.

Udenfriend, S. : Tyrosine hydroxylase. In : Second Catecholamine Symposium, Milan, 1965. Pharmacol. Rev. 18: 43, 1966.

Udenfriend, S., Zaltzman-Nirenberg, P. and Nagatsu, T. : Inhibitors of purified beef adrenal tyrosine hydroxylase. Biochem. Pharmaco1. 14: 837, 1965.

Ungher, J., Stoica, I., Sistavrica, R. : Conditionarea manifestarilor epileptiforme. Stud. Cercet. Fiziot. Neurol. 3: 233, 1952. Cited by Kreindler, A. : In : Experimental EpiTepsy. Prog. Brain. Res. 19: 79, 1965.

Vaughan-Williams, E.M. : Central nervous system effects of beta adrenergic blocking drugs. Ann. N.Y. Acad. Sci. 139: 808, 1967.

Vernadakis, A. and Woodbury, D.M. : Effect of cortisol and diphenylhydantoin (Dilantin) on spinal cord convulsions in developing rats. J. Pharmacol. Exp. Ther. 144: 316, 1964. 
Vernadakis, A. and Woodbury, D.M. : Effects of diphenylhydantoin on electroshock seizure thresholds in developing rats. J. Pharmacol. Exp. Ther. 148: 144, 1965.

Vernadakis, A. and Woodbury, D.M. : The developing animal as a model. Epilepsia 10: 163, 1969.

Vicari, E.N. : Fatal convulsive seizures in DBA mouse strains. J. Psychol: 32: 79, 1951.

Vogt, M. : The concentration of sympathin in different parts of the central nervous system under normal conditions and after the administration of drugs. J. Physiol. 123: 451, 1954.

Ward, A.A. : The hyperexcitable neuron - epilepsy. In : Radahl, K. and Issekutz, B. (Ed.) Nerve as a Tissue. Harper and Row, New York. 1966.

Weaver, J.E. and Miya, T.S. : Effect of certain ataraxic agents on mice activity. J. Pharm. Sci. 50: 910, 1961.

Weil-Malherbe, H. : Significance of glutamic acid for the metabolism of nervous tisšue. Physiol. Rev. 30: 549.

Weil-Malherbe, H. and Bone, A. : Intracellular distribution of catecholamines in the brain. Nature (Lond.) 180: 1050, 1957.

Weissman, A. and Koe, B.K. : Behavioral effects of $L-\alpha$-methyl tyrosine, an inhibitor of tyrosine hydroxylase. Life Sci. 4: 1037, 1965.

Weissman, A. and Koe, B.K. : m-Fluorotyrosine convulsions and mortality: Relationship to catecholamine and citrate metabolism. J. Pharmacol. Exp. Ther. 155: 135, 1967.

Westfa11, T.C. : Effect of beta adrenergic blocking agents on the noradrenal ine content of rat heart before and after noradrenal ine infusion. Europ. J. Pharmacol. 2: 163, 1967.

Whittaker, V.P. : Catecholamine storage particles in the central neryous system. In : Second Catecholamine Symposium, Milan. 1965. Pharmaco1. Rev. 18: 401, 1966.

Wolf, H.H. and Stock, G.A. : Utility of two conyulsant techniques as indicators of CNS excitability. J. Pharm. Sci: $\underline{55}$ : 1455, 1966.

Wolf, H.H., Swinyard, E.A. and Goodman, L.S. : Anticonvulsant properties of some N-substituted hydantoins. J. Pharm. Sci. 51: $74,1962$. 
Wood, J.D. and Watson, W.J. : Gamma-aminobutyric acid levels in the brain of rats exposed to oxygen at high pressures. Can. J. Biochem. Physiol. 41: 1907, 1963.

Wood, J.D., Watson, W.J. and Ducker, A.J. : Oxygen poisoning in various mammalian species and the possible role of gammaaminobutyric acid metabolism. J. Neurochem. 14: 1067, 1967.

Wood, J.D., Watson, W.J., Murray, G.W. : Correlation between decreases in brain gamma-aminobutyric acid levels and susceptibility to convulsions induced by hyperbaric oxygen. J. Neurochem. 16: $281,1969$.

Woodbury, D.M. : Effect of hormones on brain excitability and electrolytes. Recent Prog. Hormone Res. 10: 64, 1954.

Woodbury, D.M. : Effect of diphenylhydanto in on electrolytes and radiosodium turnover in brain and other tissues of normal, hyponatremic and postictal rats. J. Pharmacol. Exp. Ther. 115: $74,1955$.

Woodbury, D.M. : Interrelationships between ion transport mechanisms and excitatory events. Fed. Proc. 22: 31, 1963.

Woodbury, D.M. : Role of pharmacological factors in the evaluation of the anticonvulsant drugs. Epilepsia 10: 121, 1969.

Woodbury, D.M., Harley, R.E., Lewis, N.G., McArthur, M.W., Copeland, W.W., Kirschvink, J.F. and Goodman, L.S. : Effect of thyroidectomy and 6-n-propy1-2-thiouracil on brain function. $J$. Pharmacol. Exp. Ther. 106: $331,1952$.

Woodbury, D.M., Koch, A. and Vernadakis, A. : Relation between excitability and metabol ism in brain as elucidated by anticonvulsant drugs. Neurology (Minneap.). 8: Supp1. 1, 113, 1958a.

Woodbury, D.M., Rollins, L.T., Gardner, M.D., Hirschi, W.L., Hogan, J.R., Ralijison, M.L., Tanner, G.S. and Brodie, D.A. : Effects of carbon dioxide on brain excitability and electrolytes. Amer. J. Physio1: 192: 79, 1958b.

Woolley, D.E. and Timiras, P.S. : Estrous and circadian periodicity and electroshock convulsions in rats. Amer. J. Physiol. 202: $379,1962$.

Xavier, E. and Timo-Iaria, C. : On some neural effects of chlorpromazine and methamphetamine. Arch. Int. Pharmacodyn: 147: 512, 1964. 
Yeoh, P.N. : The role of endogenous catecholamines in the expression of audiogenic and electroshock seizures. M.Sc. Thesis. The Ohio State U., Columbus, Ohio. 1966.

Yeoh, P.N. and Wolf, H.H. : Effects of some adrenergic agents on low frequency electroshock seizures. J. Pharm. Sci. 47: $340,1968$.

Yen, H.C.Y., Salvatore, A.T., Silverman, A.J, and Kline, T.O. : Effect of iproniazid on anticonvul sants. Arch. Int. Pharmacodyn. 140: $631,1962$.

Yong, M.S. and Marks, G.S. : Studies of the chemical nature of the alpha-adrenergic receptor. IV. Labeling studies on nerve-free rabbit aortic strips. Biochem. Pharmacol. 18: 1619, 1969.

Zablocka, B. : Effects of autonomic agents, alone and in combination with antiepileptic drugs on electroshock seizures in rats.

Arch. Int. Pharmacodyn. 142: 533, 1963.

Zaimis, E. : Reserpine-induced circulatory failure. Nature (Lond.). 192: $521,1961$. 